\title{
Iodide as Nucleophilic Trigger in Aryne Three-Component Coupling for the Synthesis of 2-Iodobenzyl Alcohols
}

\author{
Subrata Bhattacharjee, Avishek Guin, Rahul N. Gaykar, and Akkattu T. Biju* \\ Department of Organic Chemistry, Indian Institute of Science, Bangalore-560012, \\ India
}

atbiju@iisc.ac.in

\section{Supporting Information}

$\begin{array}{ll}\text { 1. General Information } & \text { S02 }\end{array}$

2. General Procedure for the Optimization of Reaction Conditions $\quad$ S02

3. General Procedure for the Halide Triggered Aryne MCC with Aldehydes S05

4. Procedure for the Iodide triggered aryne MCC with $N$-Methylisatin $\quad \mathrm{S} 07$

5. Procedure for the Iodide Triggered Aryne $\mathrm{MCC}$ with $\mathrm{CO}_{2} \quad \mathrm{~S} 07$

$\begin{array}{ll}\text { 6. Mechanistic Experiments } & \text { S08 }\end{array}$

7. X-ray Data of 3ac and 8a $\quad$ S10

8. Synthesis and Characterization of 2-Halo Benzyl Alcohol Derivatives S13

9. Functionalization of 2-Iodobenzyl Alcohols $\quad$ S35

10. ${ }^{1} \mathrm{H}$ and ${ }^{13} \mathrm{C}$ NMR Spectra of Products $\quad$ S40 


\section{General Information}

Unless otherwise specified, all reactions were carried out under an atmosphere of nitrogen in flame-dried reaction vessels with Teflon screw caps. $25{ }^{\circ} \mathrm{C}$ Corresponds to the room temperature (rt) of the lab when the experiments were carried out. THF was freshly purified by distillation over Na-benzophenone and was transferred under nitrogen. 18-Crown-6 was recrystallized from dry $\mathrm{CH}_{3} \mathrm{CN}$ and $\mathrm{KF}, \mathrm{KI}, \mathrm{KBr}$ and $\mathrm{KCl}$ were dried by heating at $110{ }^{\circ} \mathrm{C}$ for 12 $\mathrm{h}$ and left to cool under nitrogen and stored in nitrogen filled glove-box. The 2(trimethylsilyl)phenyl trifluoromethanesulfonate 2a and the other symmetric and unsymmetrical aryne precursors were synthesized following literature procedure. ${ }^{1}$

Analytical thin layer chromatography was performed on TLC Silica gel $60 \mathrm{~F}_{254}$. Visualization was accomplished with short wave UV light or $\mathrm{KMnO}_{4}$ staining solutions followed by heating. Flash chromatography was performed on silica gel (230-400 mesh) by standard techniques eluting with Pet. Ether-EtOAc solvent system.

All compounds were fully characterized. ${ }^{1} \mathrm{H}$ and ${ }^{13} \mathrm{C}$ NMR spectra were recorded on Bruker Ultrashield spectrometer in $\mathrm{CDCl}_{3}$ as solvent. Chemical shifts $(\delta)$ are given in ppm. The residual solvent signals were used as references and the chemical shifts converted to the TMS scale $\left(\mathrm{CDCl}_{3}: \delta_{\mathrm{H}}=7.26 \mathrm{ppm}, \delta_{\mathrm{C}}=77.16 \mathrm{ppm}\right)$. Infrared (FT-IR) spectra were recorded on a Perkin Elmer Spectrum BX spectrophotometer, $v$-max in $\mathrm{cm}^{-1}$. HRMS (ESI) data were recorded on a Waters Xevo G2-XS Q-TOF instrument.

\section{General Procedure for the Optimization of the Reaction Conditions}

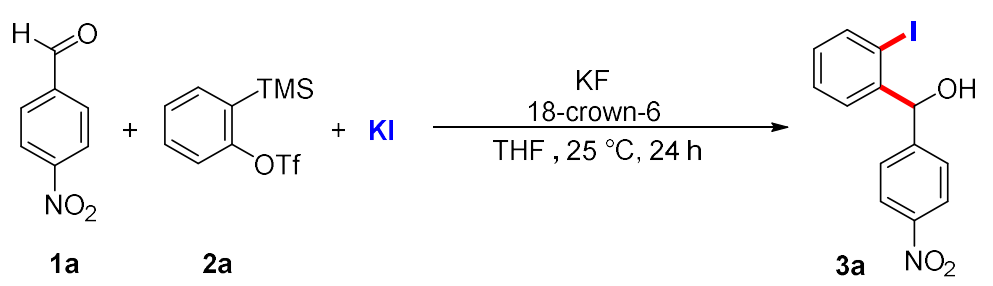

To a flame-dried screw-capped test tube equipped with a magnetic stir bar was added the KI, KF and 18-crown-6 in a glove-box. Then aldehyde 1a $(0.25 \mathrm{mmol})$ was added outside the

${ }^{1}$ (a) Sato, Y.; Tamura, T.; Kinbara, A.; Morib, M Adv. Synth. Catal. 2007, 349, 647. (b) Peña, D.; Cobas, A.; Pérez, D.; Guitián, E. Synthesis, 2002, 1454. 
glove-box under nitrogen atmosphere followed by addition of THF. To the stirring solution, aryne precursor $2 \mathbf{a}$ was added and reaction mixture was allowed to stir at $25{ }^{\circ} \mathrm{C}$ for $24 \mathrm{~h}$. After $24 \mathrm{~h}$, the reaction was quenched by adding $4 \mathrm{~N} \mathrm{HCl}(\mathrm{HCl}$ in dioxane, $0.4 \mathrm{~mL})$ and the solvent was evaporated. Yields were determined by the ${ }^{1} \mathrm{H}$ NMR analysis of the crude reaction products using $\mathrm{CH}_{2} \mathrm{Br}_{2}$ as the internal standard.

\section{Variation of the amount of Aryne precursor (2a)}
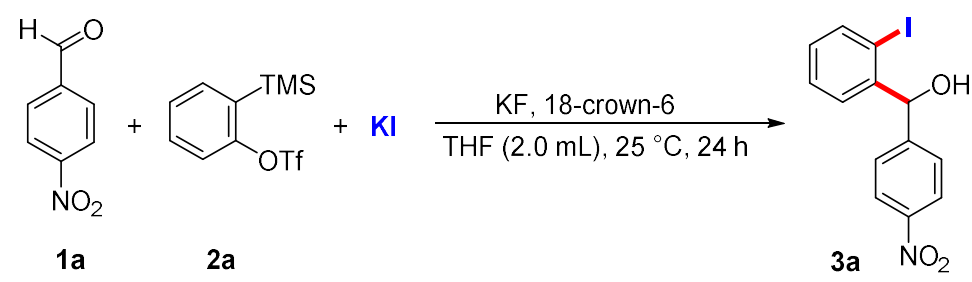

\begin{tabular}{|c|c|l|c|}
\hline entry & 2a (equiv) & \multicolumn{1}{|c|}{ F $^{-}$Source (equiv) } & Yield $^{\mathrm{a}}$ of 3a \\
\hline 1 & 1.3 & $\mathrm{KF}(2.6), 18$-crown-6 (2.6) & $52 \%$ \\
\hline 2 & 1.5 & $\mathrm{KF}(3), 18$-crown-6 (3) & $61 \%$ \\
\hline 3 & 2 & $\mathrm{KF}(4), 18$-crown-6 (4) & $64 \%$ \\
\hline 4 & 2.5 & $\mathrm{KF}(5), 18$-crown-6 (5) & $67 \%$ \\
\hline $5^{\mathrm{b}}$ & 2 & $\mathrm{CsF}$ & $18 \%$ \\
\hline 6 & 2 & $\mathrm{TBAF}$ & $<5 \%$ \\
\hline
\end{tabular}

Reaction condition: 1a $(0.25 \mathrm{mmol}), \mathrm{KI}(2$ equiv $), \mathrm{KF}\left(\mathrm{THF}(2.0 \mathrm{~mL}), 25^{\circ} \mathrm{C}\right.$ for $24 \mathrm{~h} .{ }^{\mathrm{a}}$ Yields based on the ${ }^{1} \mathrm{H}$ NMR analysis of the crude reaction products using $\mathrm{CH}_{2} \mathrm{Br}_{2}$ as the internal standard. ${ }^{\mathrm{b}}$ Reaction was performed in $2 \mathrm{~mL} \mathrm{CH}_{3} \mathrm{CN}$

\section{Variation of Iodide source}
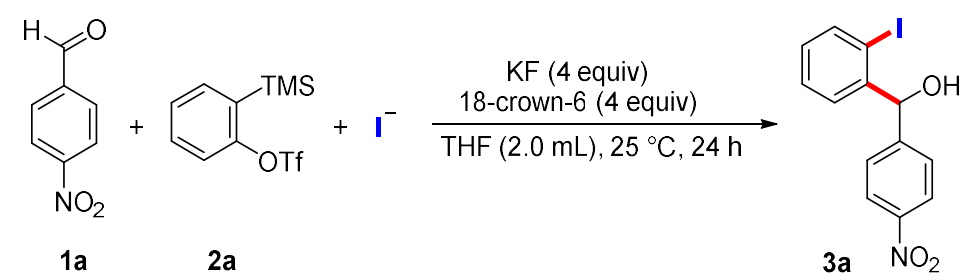

\begin{tabular}{|c|c|c|}
\hline entry & Iodide Source (equiv) & Yield $^{\mathrm{a}}$ of 3a \\
\hline 1 & KI (1.2 equiv) & $32 \%$ \\
\hline 2 & KI (2 equiv) & $64 \%$ \\
\hline 3 & KI ( 3 equiv) & $61 \%$ \\
\hline 4 & NaI (2 equiv) & $<5 \%$ \\
\hline 5 & TBAI (2 equiv) & $37 \%$ \\
\hline
\end{tabular}


Reaction condition: 1a $(0.25 \mathrm{mmol}), 2 \mathrm{a}(0.50 \mathrm{mmol}), \mathrm{KF}$ (4 equiv), 18-crown-6 (4 equiv), THF $(2.0 \mathrm{~mL}), 25{ }^{\circ} \mathrm{C}$ for $24 \mathrm{~h}$. ${ }^{\text {a }}$ Yields based on the ${ }^{1} \mathrm{H}$ NMR analysis of the crude reaction products using $\mathrm{CH}_{2} \mathrm{Br}_{2}$ as the internal standard.

\section{Variation of Temperature}

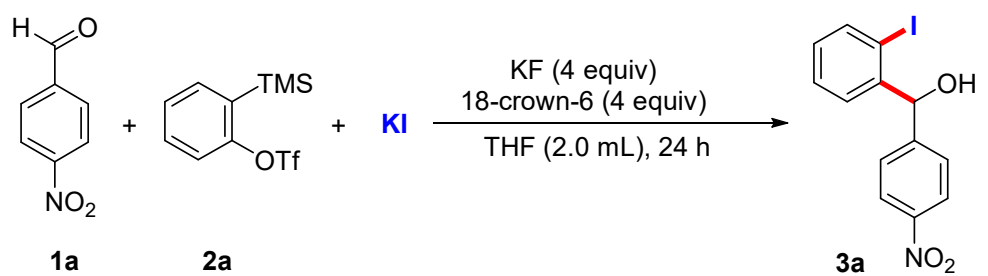

\begin{tabular}{|c|c|c|}
\hline entry & Temperature $\left({ }^{\circ} \mathbf{C}\right)$ & Yield $^{\mathrm{a}}$ of 3a \\
\hline 1 & $0{ }^{\circ} \mathrm{C}$ & $58 \%$ \\
\hline 2 & $\mathrm{rt}\left(25^{\circ} \mathrm{C}\right)$ & $64 \%$ \\
\hline 3 & $\left.40^{\circ} \mathrm{C}\right)$ & $54 \%$ \\
\hline 4 & $\left.50{ }^{\circ} \mathrm{C}\right)$ & $49 \%$ \\
\hline
\end{tabular}

Reaction condition: 1a $(0.25 \mathrm{mmol}), 2 \mathrm{a}(0.50 \mathrm{mmol}), \mathrm{KI}$ (2 equiv), KF (4 equiv), 18crown-6 (4 equiv), THF (2.0 mL), for $24 \mathrm{~h}$. ${ }^{\text {a }}$ Yields based on the ${ }^{1} \mathrm{H}$ NMR analysis of the crude reaction products using $\mathrm{CH}_{2} \mathrm{Br}_{2}$ as the internal standard.

\section{Further Optimizations}

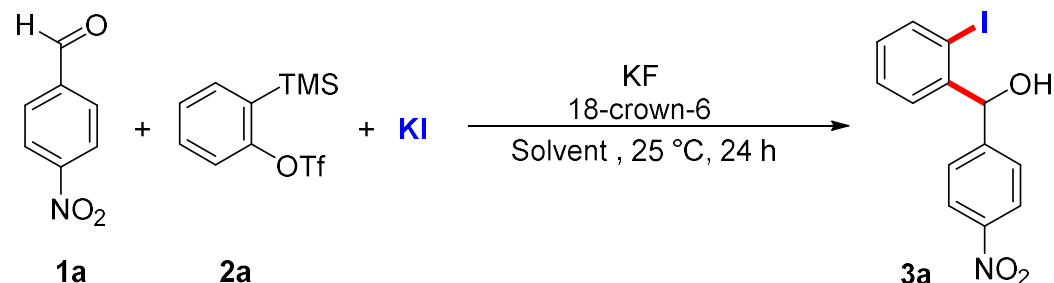

\begin{tabular}{|c|c|c|c|c|c|}
\hline entry & 2a (equiv) & F $^{-}$Source (equiv) & Solvent (mL) & KI (equiv) & Yield $^{\text {a }}$ of 3a \\
\hline 1 & 2 & KF (4), 18-crown-6 (4) & THF (2 mL) & 2 equiv & $64 \%$ \\
\hline 2 & 2 & KF (4), 18-crown-6 (4) & DME (2 mL) & 2 equiv & $53 \%$ \\
\hline 3 & 2 & KF (4), 18-crown-6 (4) & $\begin{array}{c}1,4-D i o x a n e \\
(2 \mathrm{~mL})\end{array}$ & 2 equiv & \\
\hline 4 & 2 & KF (4), 18-crown-6 (4) & THF (1 mL) & 2 equiv & $67 \%$ \\
\hline 5 & 2.5 & KF (5), 18-crown-6 (5) & THF (1 mL) & 2 equiv & $72 \%$ \\
\hline $\mathbf{6}$ & $\mathbf{2 . 5}$ & KF (5), 18-crown-6 (5) & THF (1 $\mathbf{m L})$ & 2.5 equiv & $\mathbf{7 3 \% ( 7 0 )}$ \\
\hline
\end{tabular}

Reaction condition: 1a $(0.25 \mathrm{mmol})$, for $24 \mathrm{~h} .{ }^{\text {a }}$ Yields based on the ${ }^{1} \mathrm{H}$ NMR analysis of the crude reaction products using $\mathrm{CH}_{2} \mathrm{Br}_{2}$ as the internal standard. Isolated yield in parenthesis. 


\section{General Procedure for the Halide Triggered Aryne MCC with Aldehydes}

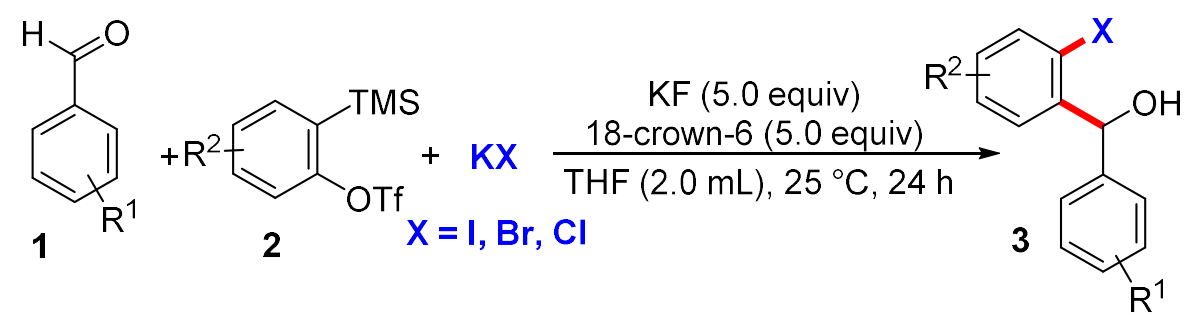

To a flame-dried screw-capped test tube equipped with a magnetic stir bar was added the $\mathrm{KX}(1.25 \mathrm{mmol}), \mathrm{KF}(0.145 \mathrm{~g}, 2.5 \mathrm{mmol})$ and 18-crown-6 (0.660 g, $2.5 \mathrm{mmol})$ in a glove box. Then aldehyde 1 ( $0.5 \mathrm{mmol})$ was added outside the glove box under nitrogen atmosphere followed by addition of THF ( $2 \mathrm{~mL})$. To the stirring solution, aryne precursor 2 (2.5 equiv) was added and reaction mixture was allowed to stir at $\mathrm{rt}$ for $24 \mathrm{~h}$. After $24 \mathrm{~h}$ the reaction was quenched by adding $4 \mathrm{~N} \mathrm{HCl}(\mathrm{HCl}$ in dioxane, $0.8 \mathrm{~mL}$ ), the solvent was evaporated and the crude residue pre-adsorbed on silica gel and purified by flash column chromatography (Pet. ether $/$ EtOAc $=90 / 10)$ on silica gel to afford the corresponding 2-halo benzyl alcohol derivatives 3 in moderate to good yields.

\section{Procedure for the $3.0 \mathrm{mmol}$ Scale Reaction for the synthesis of 3 a}
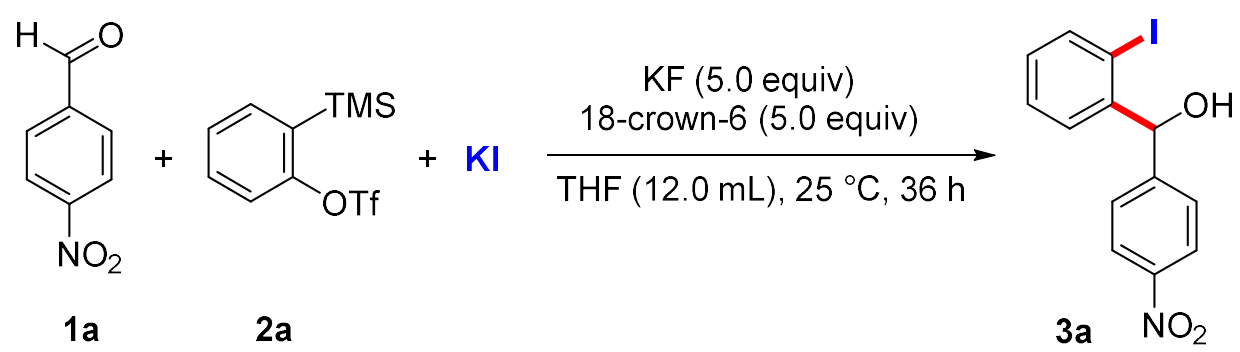

To a flame-dried screw-capped test tube equipped with a magnetic stir bar was added the $\mathrm{KI}(1.245 \mathrm{~g}, 7.5 \mathrm{mmol}), \mathrm{KF}(0.870 \mathrm{~g}, 15.0 \mathrm{mmol})$ and 18 -crown-6 (3.960 g, $15.0 \mathrm{mmol})$ in a glove-box. Then 4-nitrobenzaldehyde 1a $(0.453 \mathrm{~g}, 3.0 \mathrm{mmol})$ was added outside the glove-box under nitrogen atmosphere followed by addition of THF $(12 \mathrm{~mL})$. To the stirring solution, 2(trimethylsilyl)phenyl trifluoromethanesulfonate $\mathbf{2 a}(2.238 \mathrm{~g}, 7.5 \mathrm{mmol})$ was added and reaction mixture was allowed to stir at $\mathrm{rt}$ for $36 \mathrm{~h}$. After $36 \mathrm{~h}$, the reaction was quenched by adding $4 \mathrm{~N}$ $\mathrm{HCl}(\mathrm{HCl}$ in dioxane, $4.8 \mathrm{~mL})$ at $0{ }^{\circ} \mathrm{C}$, the solvent was evaporated and the crude residue was purified by flash column chromatography (Pet. ether $/$ EtOAc $=90 / 10)$ on silica gel to afford (2iodophenyl)(4-nitrophenyl)methanol 3a as a yellow solid (0.733 g, 69 \% yield). 


\section{Procedure for the 3.0 mmol Scale Reaction for the synthesis of $3 i$}<smiles>CC(=O)c1ccc(C(O)c2ccccc2I)cc1</smiles>

$3 \mathbf{i}$

Following the above procedure, treatment of methyl 4formylbenzoate 1i (0.493 g, $3.0 \mathrm{mmol})$, 2-(trimethylsilyl)phenyl trifluoromethanesulfonate $2 \mathrm{a}(2.238 \mathrm{~g}, 7.5 \mathrm{mmol})$ and $\mathrm{KI}(1.245 \mathrm{~g}$, $7.5 \mathrm{mmol})$ in the presence of $\mathrm{KF}(0.870 \mathrm{~g}, 15.0 \mathrm{mmol})$ and 18 -crown$6(3.960 \mathrm{~g}, 15.0 \mathrm{mmol})$ in THF $(12.0 \mathrm{~mL})$ at $25^{\circ} \mathrm{C}$ for $36 \mathrm{~h}$ followed by quenching the reaction mixture using 4N HCl ( $\mathrm{HCl}$ in dioxane, $4.8 \mathrm{~mL})$, and subsequent purification via silica gel flash column chromatography (Pet. ether $/$ EtOAc $=90 / 10$ ) of the crude reaction mixture afforded methyl-4-(hydroxy(2-iodophenyl)methyl)benzoate $3 \mathbf{i}$ as a yellow oil (0.762 g, 69\% yield).

\section{Procedure for the $3.0 \mathrm{mmol}$ Scale Reaction for the synthesis of $3 y$}<smiles>O=[N+]([O-])c1ccc(C(O)c2cc3c(cc2I)OCO3)cc1</smiles>

Following the procedure, treatment of 4-nitrobenzaldehyde 1a $(0.453$ g, $\quad 3.0 \quad \mathrm{mmol}), \quad 6-($ trimethylsilyl)benzo[d][1,3]dioxol-5-yl trifluoromethanesulfonate $2 \mathrm{c}(1.027 \mathrm{~g}, 7.5 \mathrm{mmol})$ and $\mathrm{KI}(1.245 \mathrm{~g}$, $7.5 \mathrm{mmol})$ in the presence of $\mathrm{KF}(0.870 \mathrm{~g}, 15.0 \mathrm{mmol})$ and 18 -crown$6(3.960 \mathrm{~g}, 15.0 \mathrm{mmol})$ in THF $(12.0 \mathrm{~mL})$ at $25^{\circ} \mathrm{C}$ for $36 \mathrm{~h}$ followed by quenching the reaction mixture using $4 \mathrm{~N} \mathrm{HCl}(\mathrm{HCl}$ in dioxane, $4.8 \mathrm{~mL})$, and subsequent purification via silica gel flash column chromatography (Pet. ether $/$ EtOAc $=90 / 10)$ of the crude reaction mixture afforded (6iodobenzo[d][1,3]dioxol-5-yl)(4-nitrophenyl)methanol $\mathbf{3 y}$ as a yellow oil $(0.850 \mathrm{~g}, 71 \%$ yield $)$.

\section{Procedure for the 3.0 mmol Scale Reaction for the synthesis of 3ae}<smiles>O=[N+]([O-])c1ccc(C(O)c2ccccc2Br)cc1</smiles>

3ae

Following the procedure, treatment of 4-nitrobenzaldehyde 1a $(0.453 \mathrm{~g}$, $3.0 \mathrm{mmol}), 2$-(trimethylsilyl)phenyl trifluoromethanesulfonate $\mathbf{2 a}(2.238$ $\mathrm{g}, 7.5 \mathrm{mmol})$ and $\mathrm{KBr}(0.893 \mathrm{~g}, 7.5 \mathrm{mmol})$ in the presence of $\mathrm{KF}(0.870$ $\mathrm{g}, 15.0 \mathrm{mmol})$ and 18 -crown-6 (3.960 g, $15.0 \mathrm{mmol})$ in THF $(12.0 \mathrm{~mL})$ at $25{ }^{\circ} \mathrm{C}$ for $36 \mathrm{~h}$ followed by quenching the reaction mixture using $4 \mathrm{~N} \mathrm{HCl}(\mathrm{HCl}$ in dioxane, 4.8 $\mathrm{mL}$ ), and subsequent purification via silica gel flash column chromatography (Pet. ether /EtOAc $=90 / 10)$ of the crude reaction mixture afforded (2-bromophenyl)(4-nitrophenyl)methanol 3ae as a yellow solid $(0.691 \mathrm{~g}, 75 \%$ yield $)$. 


\section{Procedure for the Iodide triggered aryne MCC with $N$-Methylisatin}

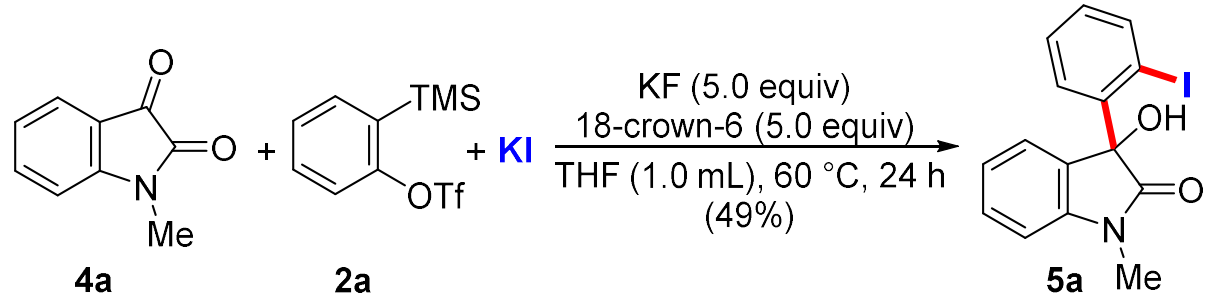

To a flame-dried screw-capped test tube equipped with a magnetic stir bar was added the $\mathrm{KI}(0.104 \mathrm{~g}, 0.625 \mathrm{mmol}), \mathrm{KF}(0.073 \mathrm{~g}, 1.25 \mathrm{mmol})$ and 18 -crown-6 (0.330 g, $1.25 \mathrm{mmol})$ in a glove-box. Then 1-methylindoline-2,3-dione 4a $(0.040,0.25 \mathrm{mmol})$ was added outside the glovebox under nitrogen atmosphere followed by addition of THF $(1 \mathrm{~mL})$. To the stirring solution, aryne precursor $\mathbf{2 a}(0.186 \mathrm{~g}, 152 \mu \mathrm{L}, 0.625 \mathrm{mmol})$ was added and reaction mixture was allowed to stir at $60{ }^{\circ} \mathrm{C}$ for $24 \mathrm{~h}$. After $24 \mathrm{~h}$, the reaction was quenched by adding $4 \mathrm{~N} \mathrm{HCl}(\mathrm{HCl}$ in dioxane, $0.4 \mathrm{~mL}$ ), the solvent was evaporated and the crude residue pre-adsorbed on silica gel and purified by flash column chromatography (Pet. ether $/$ EtOAc $=70 / 30)$ afforded 3-hydroxy-3(2-iodophenyl)-1-methylindolin-2-one 5a in 49\% yield.

\section{Procedure for the Iodide triggered aryne $\mathrm{MCC}$ with $\mathrm{CO}_{2}$}

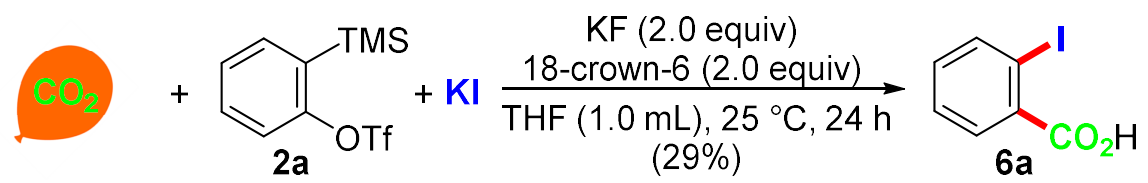

To a flame-dried screw-capped test tube equipped with a magnetic stir bar was added the $\mathrm{KI}(0.104 \mathrm{~g}, 0.625 \mathrm{mmol}), \mathrm{KF}(0.029 \mathrm{~g}, 0.5 \mathrm{mmol})$ and 18-crown-6 (0.132 g, $0.5 \mathrm{mmol})$ inside the glove-box. Then the screw-capped test tube was evacuated and backfilled with $\mathrm{CO}_{2}$ (balloon) for four times followed by the addition of THF $(1 \mathrm{~mL})$. Then aryne precursor $(0.075 \mathrm{~g}, 0.25$ mmol) was added, the reaction mixture was allowed to stir for $24 \mathrm{~h}$ under the atmosphere of $\mathrm{CO}_{2}$ (balloon). After $24 \mathrm{~h}$ the reaction mixture was quenched with cold water and washed with dichloromethane three times. Then $1 \mathrm{~N} \mathrm{HCl}$ was added to the water layer and extracted with ethyl acetate (four times). Then solvent was evaporated to afford 2-iodobenzoic acid in 29\% yield. 


\section{Mechanistic Experiments}

\section{Deuterium Labeling Experiment}

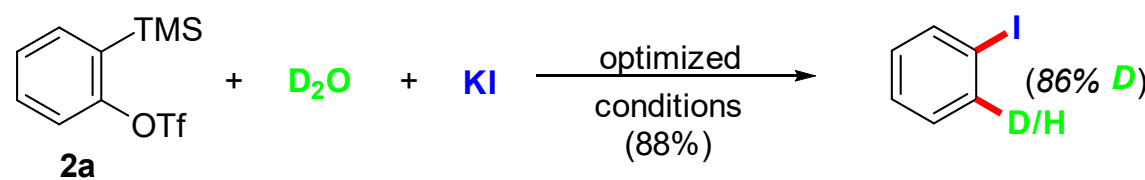

To a flame-dried screw-capped test tube equipped with a magnetic stir bar was added the $\mathrm{KI}(0.104 \mathrm{~g}, 0.625 \mathrm{mmol}), \mathrm{KF}(0.073 \mathrm{~g}, 1.25 \mathrm{mmol})$ and 18 -crown-6 $(0.330 \mathrm{~g}, 1.25 \mathrm{mmol})$ in a glove-box. Then $\mathrm{D}_{2} \mathrm{O}(0.005 \mathrm{~g}, 4.5 \mu \mathrm{L}, 0.25 \mathrm{mmol})$ was added outside the glove-box under nitrogen atmosphere followed by addition of THF $(1 \mathrm{~mL})$. To the stirring solution, aryne precursor $2 \mathrm{a}(0.186 \mathrm{~g}, 152 \mu \mathrm{L}, 0.625 \mathrm{mmol})$ was added and reaction mixture was allowed to stir at $\mathrm{rt}$ for $24 \mathrm{~h}$. After $24 \mathrm{~h}$ the solvent was evaporated and the crude residue pre-adsorbed on silica gel and purified by flash column chromatography (Pet. ether) on silica gel to afford the iodobenzene 5 in $88 \%$ yield with $86 \%$ D incorporation at the 2-position.

${ }^{1} \mathrm{H}$ NMR spectrum of Iodobenzene-D (86\% D)

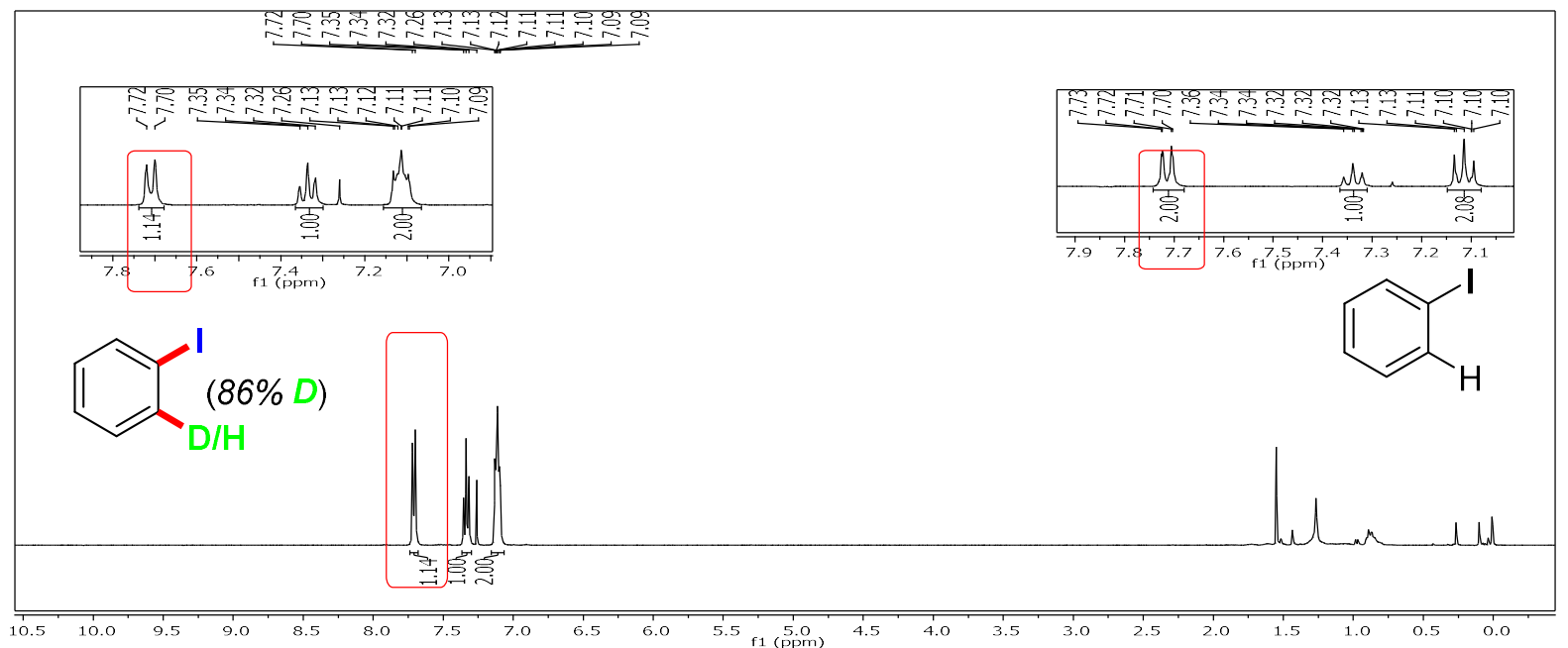

Deuterium Labeling Experiment in presence of aldehyde:<smiles>C[13C](=O)[O-]</smiles> 
To a flame-dried screw-capped test tube equipped with a magnetic stir bar was added the $\mathrm{KI}(0.104 \mathrm{~g}, 0.625 \mathrm{mmol}), \mathrm{KF}(0.073 \mathrm{~g}, 1.25 \mathrm{mmol})$ and 18 -crown-6 $(0.330 \mathrm{~g}, 1.25 \mathrm{mmol})$ in a glove-box. Then 4-nitrobenzaldehyde $1 \mathrm{a}(0.038 \mathrm{~g}, 0.25 \mathrm{mmol})$ and $\mathrm{D}_{2} \mathrm{O}(0.005 \mathrm{~g}, 4.5 \mu \mathrm{L}, 0.25$ mmol) was added outside the glove-box under nitrogen atmosphere followed by addition of THF $(1 \mathrm{~mL})$. To the stirring solution, aryne precursor $2 \mathrm{a}(0.186 \mathrm{~g}, 152 \mu \mathrm{L}, 0.625 \mathrm{mmol})$ was added and reaction mixture was allowed to stir at $\mathrm{rt}$ for $24 \mathrm{~h}$. After $24 \mathrm{~h}$ the reaction was quenched by adding $4 \mathrm{~N} \mathrm{HCl}(\mathrm{HCl}$ in dioxane, $0.4 \mathrm{~mL})$, the solvent was evaporated and the crude residue preadsorbed on silica gel and purified by flash column chromatography (Pet. ether $/$ EtOAc $=90 / 10$ ) on silica gel to afford the product 3a in (0.036 g) 40\% yield along with the iodobenzene with $90 \%$ D incorporation at the 2-position in ( $0.024 \mathrm{~g}) 49 \%$ yield.

\section{${ }^{1} \mathrm{H}$ NMR spectrum of Iodobenzene-D $(90 \% \mathrm{D})$}

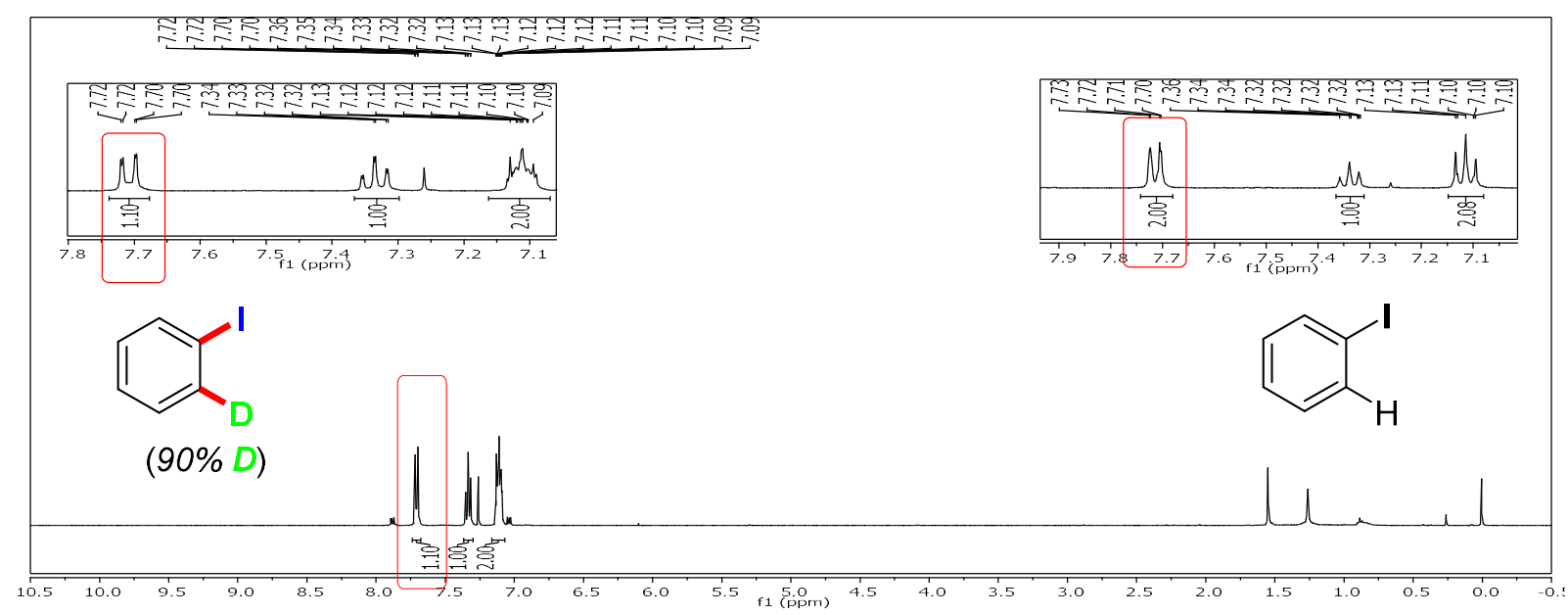

\section{Deuterium Labeling Experiment with excess $\mathrm{D}_{2} \mathrm{O}$ :}

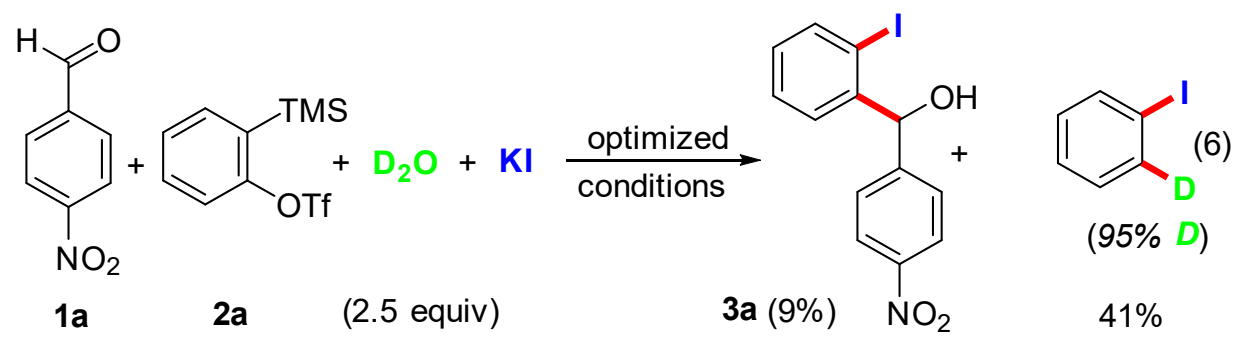

To a flame-dried screw-capped test tube equipped with a magnetic stir bar was added the $\mathrm{KI}(0.104 \mathrm{~g}, 0.625 \mathrm{mmol}), \mathrm{KF}(0.073 \mathrm{~g}, 1.25 \mathrm{mmol})$ and 18-crown-6 (0.330 g, $1.25 \mathrm{mmol})$ in a 
glove-box. Then 4-nitrobenzaldehyde 1a $(0.038 \mathrm{~g}, 0.25 \mathrm{mmol})$ and $\mathrm{D}_{2} \mathrm{O}(0.013 \mathrm{~g}, 11.5 \mu \mathrm{L}$, $0.625 \mathrm{mmol}$ ) was added outside the glove-box under nitrogen atmosphere followed by addition of THF ( $1 \mathrm{~mL})$. To the stirring solution, aryne precursor $2 \mathrm{a}(0.186 \mathrm{~g}, 152 \mu \mathrm{L}, 0.625 \mathrm{mmol})$ was added and reaction mixture was allowed to stir at $\mathrm{rt}$ for $24 \mathrm{~h}$. After $24 \mathrm{~h}$ the reaction was quenched by adding $4 \mathrm{~N} \mathrm{HCl}(\mathrm{HCl}$ in dioxane, $0.4 \mathrm{~mL})$, the solvent was evaporated and the crude residue pre-adsorbed on silica gel and purified by flash column chromatography (Pet. ether $/$ EtOAc $=90 / 10)$ on silica gel to afford the product 3a $(0.008 \mathrm{~g}) 9 \%$ yield along with the iodobenzene with $95 \% \mathrm{D}$ incorporation at the 2-position in $(0.052 \mathrm{~g}) 41 \%$ yield.

\section{${ }^{1}$ H NMR spectrum of Iodobenzene-D (95\% D)}

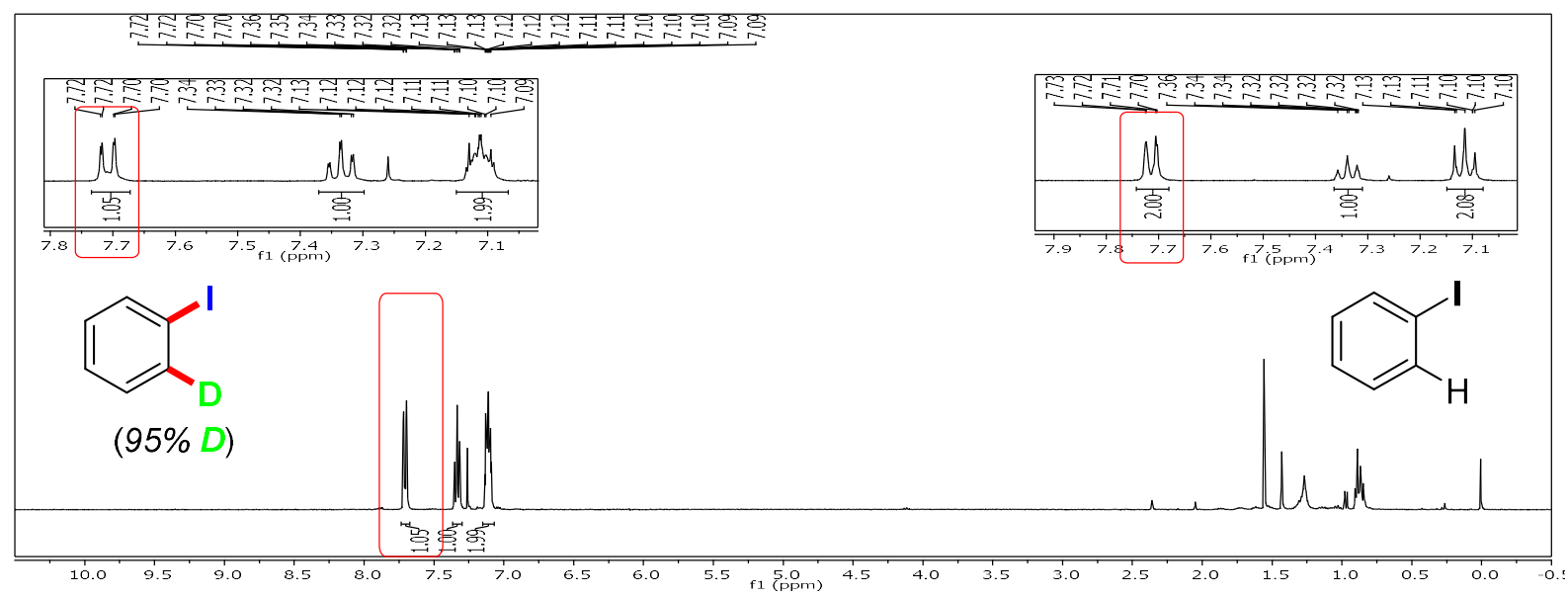

\section{X-ray Data of 3ac and 8a}

\section{Data of $3 a c$}

Single crystal of 3ac (recrystallized from $\mathrm{CH}_{2} \mathrm{Cl}_{2}$ at $25{ }^{\circ} \mathrm{C}$ ) was mounted and the diffraction data was collected at $293 \mathrm{~K}$ on a Bruker SMART APEX CCD diffractometer using SMART/SAINT software. Intensity data were collected using graphite-monochromatized Mo-Ka radiation $(71.073 \mathrm{pm})$. The structure was solved by direct methods using the SHELX-972 and refined by full-matrix least-squares on F2. Empirical absorption corrections were applied with SADABS. ${ }^{3}$ All Non-hydrogen atoms were refined anisotropically and hydrogen atoms were included in geometric positions. Structure was drawn using Olex-2 and ORTEP-3. The crystallographic refinement parameters are given below:

\footnotetext{
${ }^{2}$ G. M. SHELXL-2013 Sheldrick, University of Göttingen: Göttingen, Germany, 2014.

${ }^{3}$ G. M. Sheldrick, SADABS, University of Göttingen, Göttingen, Germany, 1999.
} 
CCDC

Identification code

Empirical formula

Formula weight

Temperature/K

Crystal system

Space group

$\mathrm{a} / \AA$

$\mathrm{b} / \AA \AA$

$\mathrm{c} / \AA ̊$

$\alpha /^{\circ}$

$\beta /^{\circ}$

$\gamma /{ }^{\circ}$

Volume $/ \AA^{3}$

Z

$\rho_{\text {calc }} \mathrm{g} / \mathrm{cm}^{3}$

$\mu / \mathrm{mm}^{-1}$

$\mathrm{F}(000)$

Crystal size $/ \mathrm{mm}^{3}$

Radiation

CCDC 1905768

$3 a c$

$\mathrm{C}_{14} \mathrm{H}_{12} \mathrm{INO}_{4}$

385.15

293(2) K

Triclinic

P -1

11.2336(5)

$11.3868(6)$

12.1898(5)

93.014(4)

106.905(4)

108.610(4)

$1395.58(12)$

4

1.833

2.307

752

$0.263 \times 0.251 \times 0.238$

$\operatorname{MoK} \alpha(\lambda=0.71073)$

Theta range for data collection/ ${ }^{\circ} 1.769$ to 27.484

$\begin{array}{ll}\text { Index ranges } & -14 \leq \mathrm{h} \leq 14,-14 \leq \mathrm{k} \leq 14,-15 \leq 1 \leq 15 \\ \text { Reflections collected } & 20842 \\ \text { Independent reflections } & 6405\left[\mathrm{R}_{\text {int }}=0.0482\right] \\ \text { Data/restraints/parameters } & 6405 / 0 / 377 \\ \text { Goodness-of-fit on } \mathrm{F}^{2} & 1.033 \\ \text { Final } \mathrm{R} \text { indexes [I }>=2 \sigma(\mathrm{I})] & \mathrm{R}_{1}=0.0399, \mathrm{wR}_{2}=0.0861 \\ \text { Final } \mathrm{R} \text { indexes [all data] } & \mathrm{R}_{1}=0.0538, \mathrm{wR}_{2}=0.0965 \\ \text { Largest diff. peak/hole / e } \AA^{-3} & 1.057 /-1.330\end{array}$

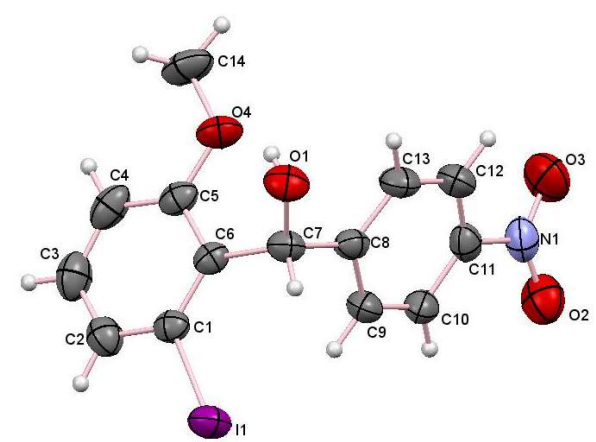

Ortep Diagram of 3ac (thermal ellipsoids are shown with 50\% probability). 
CCDC 1905768 (3ac) contains the supplementary crystallographic data for this paper. These data can be obtained free of charge from Data Centre via www.ccdc.cam.ac.uk/data request/cif

\section{Data of $8 a$}

Single crystal of $\mathbf{8 a}$ (recrystallized from $\mathrm{CH}_{2} \mathrm{Cl}_{2}$ at $25{ }^{\circ} \mathrm{C}$ ) was mounted and the diffraction data was collected at $293 \mathrm{~K}$ on a Bruker SMART APEX CCD diffractometer using SMART/SAINT software. Intensity data were collected using graphite-monochromatized Mo-Ka radiation $(71.073 \mathrm{pm})$. The structure was solved by direct methods using the SHELX-972 and refined by full-matrix least-squares on F2. Empirical absorption corrections were applied with SADABS. ${ }^{3}$ All Non-hydrogen atoms were refined anisotropically and hydrogen atoms were included in geometric positions. Structure was drawn using Olex-2 and ORTEP-3. The crystallographic refinement parameters are given below:

CCDC

Identification code

Empirical formula

Formula weight

Temperature/K

Crystal system

Space group

$\mathrm{a} / \AA$

$\mathrm{b} / \AA$

$\mathrm{c} / \AA$

$\alpha /^{\circ}$

$\beta /{ }^{\circ}$

$\gamma /{ }^{\circ}$

Volume $/ \AA^{3}$

Z

$\rho_{\text {calc }} \mathrm{g} / \mathrm{cm}^{3}$

$\mu / \mathrm{mm}^{-1}$

$\mathrm{F}(000)$

Radiation

Index ranges

Reflections collected

Independent reflections
CCDC 1911771

$8 a$

$\mathrm{C}_{21} \mathrm{H}_{15} \mathrm{NO}_{3}$

329.355

293(2) K

Monoclinic

$\mathrm{C} 2 / \mathrm{c}$

19.8644(17)

13.8976(11)

$12.4126(8)$

90 (4)

102.483 (4)

90 (4)

$3345.7(5)$

4

1.308

0.088

1376

$\operatorname{MoK} \alpha(\lambda=0.71073)$

$-25 \leq \mathrm{h} \leq 25,-18 \leq \mathrm{k} \leq 17,-16 \leq 1 \leq 16$

15233

$3840[\mathrm{R}(\mathrm{int})=0.0844]$ 


$\begin{array}{ll}\text { Data/restraints/parameters } & 3840 / 0 / 234 \\ \text { Goodness-of-fit on } \mathrm{F}^{2} & 1.029 \\ \text { Final R indexes }[\mathrm{I}>=2 \sigma(\mathrm{I})] & \mathrm{R}_{1}=0.0781, \mathrm{wR}_{2}=0.1605 \\ \text { Final R indexes [all data] } & \mathrm{R}_{1}=0.1650, \mathrm{wR}_{2}=0.2154 \\ \text { Largest diff. peak/hole } / \mathrm{e} \AA^{-3} 0.150 /-0.206\end{array}$

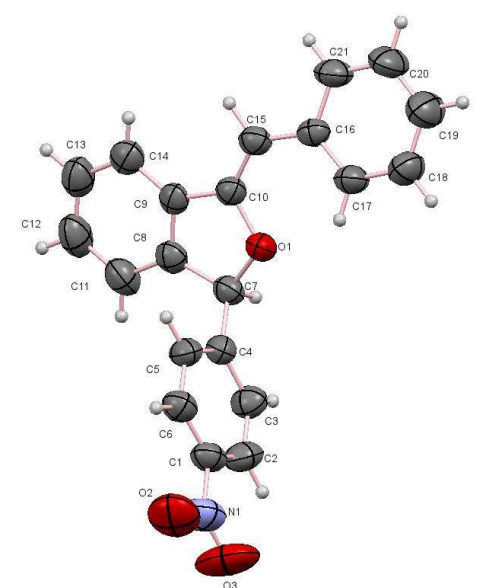

Ortep Diagram of 8a (thermal ellipsoids are shown with 50\% probability).

CCDC 1911771 (8a) contains the supplementary crystallographic data for this paper. These data can be obtained free of charge from Data Centre via $\underline{w w w . c c d c . c a m . a c . u k / d a t a \_r e q u e s t / c i f}$

\section{Synthesis and Characterization of 2-HaloBenzyl Alcohol Derivatives}

\section{(2-Iodophenyl)(4-nitrophenyl)methanol (3a)}<smiles>O=[N+]([O-])c1ccc(C(O)c2ccccc2I)cc1</smiles>

3a

Following the general procedure, treatment of 4-nitrobenzaldehyde 1a (0.038 g, $0.25 \mathrm{mmol}), 2$-(trimethylsilyl)phenyl trifluoromethanesulfonate 2a $(0.186 \mathrm{~g}, 152 \mu \mathrm{L}, 0.625 \mathrm{mmol})$ and $\mathrm{KI}(0.104 \mathrm{~g}, 0.625 \mathrm{mmol})$ in the presence of KF $(0.073 \mathrm{~g}, 1.25 \mathrm{mmol})$ and 18-crown-6 (0.330 g, 1.25 $\mathrm{mmol})$ in THF $(1.0 \mathrm{~mL})$ at $25^{\circ} \mathrm{C}$ for $24 \mathrm{~h}$ followed by quenching the reaction mixture using $4 \mathrm{~N}$ $\mathrm{HCl}(\mathrm{HCl}$ in dioxane, $0.8 \mathrm{~mL}$ ), and subsequent purification via silica gel flash column chromatography (Pet. ether $/$ EtOAc $=90 / 10$ ) of the crude reaction mixture afforded (2iodophenyl)(4-nitrophenyl)methanol 3a as a yellow solid (0.062 g, $70 \%$ yield).

$\boldsymbol{R}_{\mathbf{f}}($ Pet. ether $/$ EtOAc $=85 / 15): 0.40,{ }^{1} \mathbf{H}$ NMR (400 MHz, $\left.\mathbf{C D C l}_{3}\right) \delta 8.17(\mathrm{~d}, J=8.7 \mathrm{~Hz}, 2 \mathrm{H})$, $7.85(\mathrm{~d}, J=8.2 \mathrm{~Hz}, 1 \mathrm{H}), 7.60(\mathrm{~d}, J=8.8 \mathrm{~Hz}, 2 \mathrm{H}), 7.39-7.34(\mathrm{~m}, 2 \mathrm{H}), 7.04-7.00(\mathrm{~m}, 1 \mathrm{H}), 6.18$ (s, 
1H), 2.75 (s, 1H). ${ }^{13} \mathbf{C}$ NMR (100 MHz, $\left.\mathbf{C D C l}_{3}\right) \delta$ 149.44, 147.42, 144.68, 139.92, 130.26, 129.13, 128.66, 127.86, 123.78, 98.72, 78.28. HRMS (ESI) calculated $[\mathrm{M}-\mathrm{OH}]^{+}$for $\mathrm{C}_{13} \mathrm{H}_{9} \mathrm{INO}_{2}$ : 337.9672, found: 337.9678. FTIR (cm-1) 3400, 3074, 1601, 1718, 1598, 1517, 1008, 862.

\section{(2-Iodophenyl)(phenyl)methanol (3b) ${ }^{4}$}<smiles>OC(c1ccccc1)c1ccccc1I</smiles>

$3 b$

Following the general procedure, treatment of benzaldehyde $\mathbf{1 b}(0.027 \mathrm{~g}$, $0.026 \mu \mathrm{L}, 0.25 \mathrm{mmol}), 2$-(trimethylsilyl)phenyl trifluoromethanesulfonate $\mathbf{2 a}$ $(0.186 \mathrm{~g}, 152.0 \mu \mathrm{L}, 0.625 \mathrm{mmol})$ and $\mathrm{KI}(0.104 \mathrm{~g}, 0.625 \mathrm{mmol})$ in the presence of KF $(0.073 \mathrm{~g}, 1.25 \mathrm{mmol})$ and 18-crown-6 (0.330 g, $1.25 \mathrm{mmol})$ in THF $(1.0 \mathrm{~mL})$ at $25^{\circ} \mathrm{C}$ for $24 \mathrm{~h}$ followed by quenching the reaction mixture using $4 \mathrm{~N} \mathrm{HCl}(\mathrm{HCl}$ in dioxane, $0.4 \mathrm{~mL}$ ), and subsequent purification via silica gel flash column chromatography (Pet. ether $/$ EtOAc $=90 / 10)$ of the crude reaction mixture afforded (2Iodophenyl)(phenyl)methanol $\mathbf{3 b}$ as a yellow oil ( $0.021 \mathrm{~g}, 27 \%$ yield).

$\boldsymbol{R}_{\mathbf{f}}($ Pet. ether $/$ EtOAc $=85 / 15): 0.45 ;{ }^{1} \mathbf{H}$ NMR (400 MHz, $\left.\mathbf{C D C l}_{3}\right) \delta 7.84\left(\mathrm{dd}, J_{l}=7.9, J_{2}=1.1\right.$ $\mathrm{Hz}, 1 \mathrm{H}), 7.53\left(\mathrm{dd}, J_{1}=7.8, J_{2}=1.6 \mathrm{~Hz}, 1 \mathrm{H}\right), 7.43-7.40(\mathrm{~m}, 2 \mathrm{H}), 7.38-7.27(\mathrm{~m}, 4 \mathrm{H}), 6.99\left(\mathrm{td}, J_{1}=\right.$ 7.7, $\left.J_{2}=1.7 \mathrm{~Hz}, 1 \mathrm{H}\right), 6.07(\mathrm{~d}, J=3.4,1 \mathrm{H}), 2.38(\mathrm{~d}, J=3.7,1 \mathrm{H}) .{ }^{13} \mathbf{C} \mathbf{N M R}\left(\mathbf{1 0 0} \mathbf{M H z}, \mathbf{C D C l}_{3}\right) \delta$ $145.49,142.24,139.70,129.59,128.73,128.63,128.51,127.93,127.36,98.79,79.15$. HRMS (ESI) calculated $[\mathrm{M}-\mathrm{OH}]+$ for $\mathrm{C}_{13} \mathrm{H}_{10} \mathrm{I}:$ 292.9822, found: 298.9829. FTIR (cm $^{-}$ 1) $3319,3059,2365,1586,1181,1006$.

\section{(4-Bromophenyl)(2-iodophenyl)methanol (3c)}<smiles>OC(c1ccc(Br)cc1)c1ccccc1I</smiles>

$3 c$

Following the general procedure, treatment of 4-bromobenzaldehyde 1c (0.093 g, $0.5 \mathrm{mmol}), 2$-(trimethylsilyl)phenyl trifluoromethanesulfonate 2a $(0.373 \mathrm{~g}, 303 \mu \mathrm{L}, 1.25 \mathrm{mmol})$ and $\mathrm{KI}(0.208 \mathrm{~g}, 1.25 \mathrm{mmol})$ in the presence of KF (0.145 g, $2.5 \mathrm{mmol})$ and 18-crown-6 (0.660 g, $2.5 \mathrm{mmol})$ in THF $(2.0 \mathrm{~mL})$ at $25^{\circ} \mathrm{C}$ for $24 \mathrm{~h}$ followed by quenching the reaction mixture using $4 \mathrm{~N} \mathrm{HCl}(\mathrm{HCl}$ in dioxane, $0.8 \mathrm{~mL}$ ), and subsequent purification via silica gel flash column chromatography (Pet. ether $/$ EtOAc $=90 / 10)$ of the crude reaction mixture afforded (4-bromophenyl)(2iodophenyl)methanol $\mathbf{3 c}$ as a yellow oil $(0.132 \mathrm{~g}, 67 \%$ yield $)$.

\footnotetext{
${ }^{4}$ Cvengroš, J., Stolz, D., Togni, A. Synthesis 2009, 16, 2818.
} 
$\boldsymbol{R}_{\mathbf{f}}($ Pet. ether $/$ EtOAc $=85 / 15): 0.5 ;{ }^{1} \mathbf{H}$ NMR (400 MHz, $\left.\mathbf{C D C l}_{3}\right) \delta 7.83(\mathrm{~d}, J=8.0 \mathrm{~Hz}, 1 \mathrm{H})$, 7.46-7.44 (m, 3H), 7.36(t, $J=7.2 \mathrm{~Hz}, 1 \mathrm{H}), 7.27(\mathrm{~d}, J=8.3 \mathrm{~Hz}, 2 \mathrm{H}), 7.00\left(\mathrm{td}, J_{1}=7.6 \mathrm{~Hz}, J_{2}=\right.$ $1.6 \mathrm{~Hz} 1 \mathrm{H}), 6.00$ (s, 1H), 2.61 (bs, 1H). ${ }^{13} \mathbf{C}$ NMR (100 MHz, CDCl $\left.\mathbf{3}\right) \delta 145.06,141.19,139.75$, 131.68, 129.80, 129.02, 128.83, 128.39, 121.85, 98.68, 78.50. HRMS (ESI) calculated [M-OH $]^{+}$ for $\mathrm{C}_{13} \mathrm{H}_{9} \mathrm{BrI}$ : 370.8927, found: 370.8933. Isotopic Mass: [M-OH+2] 372.8914 . FTIR (cm 1) $3350,2923,1639,1178,1007,853$.

\section{(4-Chlorophenyl)(2-iodophenyl)methanol (3d)}<smiles>OC(c1ccc(Cl)cc1)c1ccccc1I</smiles>

$3 d$

Following the general procedure, treatment of 4-chlorobenzaldehyde 1d (0.035 g, $0.25 \mathrm{mmol}), 2$-(trimethylsilyl)phenyl trifluoromethanesulfonate 2a $(0.186 \mathrm{~g}, 152.0 \mu \mathrm{L}, 0.625 \mathrm{mmol})$ and $\mathrm{KI}(0.104 \mathrm{~g}, 0.625 \mathrm{mmol})$ in the presence of $\mathrm{KF}(0.073 \mathrm{~g}, 1.25 \mathrm{mmol})$ and 18-crown-6 (0.330 g, 1.25 mmol) in THF $(1.0 \mathrm{~mL})$ at $25^{\circ} \mathrm{C}$ for $24 \mathrm{~h}$ followed by quenching the reaction mixture using $4 \mathrm{~N}$ $\mathrm{HCl}(\mathrm{HCl}$ in dioxane, $0.4 \mathrm{~mL}$ ), and subsequent purification via silica gel flash column chromatography (Pet. ether $/$ EtOAc $=90 / 10)$ of the crude reaction mixture afforded (4chlorophenyl)(2-iodophenyl)methanol 3d as a yellow oil (0.042 g, 49\% yield).

$\boldsymbol{R}_{\mathbf{f}}($ Pet. ether $/$ EtOAc $=85 / 15): 0.45 ;{ }^{1} \mathbf{H}$ NMR (400 MHz, $\left.\mathbf{C D C l}_{3}\right) \delta 7.84\left(\mathrm{dd}, J_{1}=7.9 \mathrm{~Hz}, J_{2}\right.$ $=1.1 \mathrm{~Hz}, 1 \mathrm{H}), 7.47\left(\mathrm{dd}, J_{1}=7.8 \mathrm{~Hz}, J_{2}=1.6 \mathrm{~Hz}, 1 \mathrm{H}\right), 7.39-7.29(\mathrm{~m}, 5 \mathrm{H}), 7.00\left(\mathrm{td}, J_{1}=7.6\right.$, $\left.J_{2}=1.7 \mathrm{~Hz}, 1 \mathrm{H}\right), 6.03$ (s, 1H), 2.47 (bs, 1H). ${ }^{13} \mathbf{C}$ NMR (100 MHz, CDCl $) \delta 145.13,140.67$, 139.77, 133.68, 129.81, 128.85, 128.75, 128.71, 128.39, 98.67, 78.49. HRMS (ESI) calculated $[\mathrm{M}-\mathrm{OH}]^{+}$for $\mathrm{C}_{13} \mathrm{H}_{9} \mathrm{ClI}: 326.9432$, found: 326.9439. Isotopic Mass: [M-OH+2] $]^{+}$328.9413. FTIR $\left(\mathbf{c m}^{-1}\right)$ 3337, 2923, 1641, 1489, 1009, 855.

\section{(4-Fluorophenyl)(2-iodophenyl)methanol (3e)}

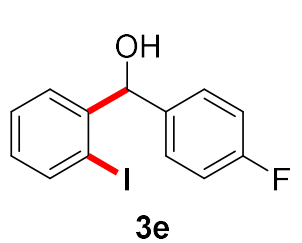

Following the general procedure, treatment of 4-fluorobenzaldehyde 1e $(0.062 \mathrm{~g}, \quad 53.6 \quad \mu \mathrm{L}, \quad 0.5 \quad \mathrm{mmol}), \quad$ 2-(trimethylsilyl)phenyl trifluoromethanesulfonate $2 \mathbf{a}(0.373 \mathrm{~g}, 303 \mu \mathrm{L}, 1.25 \mathrm{mmol})$ and $\mathrm{KI}(0.208 \mathrm{~g}$, $1.25 \mathrm{mmol})$ in the presence of $\mathrm{KF}(0.145 \mathrm{~g}, 2.5 \mathrm{mmol})$ and 18-crown-6 $(0.660 \mathrm{~g}, 2.5 \mathrm{mmol})$ in THF $(2.0 \mathrm{~mL})$ at $25^{\circ} \mathrm{C}$ for $24 \mathrm{~h}$ followed by quenching the reaction mixture using $4 \mathrm{~N} \mathrm{HCl}(\mathrm{HCl}$ in dioxane, $0.8 \mathrm{~mL})$, and subsequent purification via silica gel flash 
column chromatography (Pet. ether $/$ EtOAc $=90 / 10)$ of the crude reaction mixture afforded (4fluorophenyl)(2-iodophenyl)methanol 3e as a white solid (0.074 g, 47\% yield).

$\boldsymbol{R}_{\mathbf{f}}($ Pet. ether $/$ EtOAc $=85 / 15): 0.58 ;{ }^{1} \mathbf{H}$ NMR (400 $\left.\mathbf{M H z}, \mathbf{C D C l}_{3}\right) \delta 7.84\left(\mathrm{dd}, J_{1}=8.0 \mathrm{~Hz}, J_{2}=\right.$ $0.9 \mathrm{~Hz}, 1 \mathrm{H}), 7.50$ (dd, $\left.J_{l}=7.8 \mathrm{~Hz}, J_{2}=1.5 \mathrm{~Hz}, 1 \mathrm{H}\right), 7.40-7.34(\mathrm{~m}, 3 \mathrm{H}), 7.04-6.98$ (m, 3H), 6.01 (s, 1H), 2.60 (bs, 1H). ${ }^{13} \mathbf{C}$ NMR (100 MHz, $\left.\mathbf{C D C l}_{3}\right) \delta 162.35$ (d, $\left.J=246.2 \mathrm{~Hz}\right), 145.28,139.74$, $137.94(\mathrm{~d}, J=3.2 \mathrm{~Hz}), 129.68,129.15(\mathrm{~d}, J=8.2 \mathrm{~Hz}), 128.77,128.27,115.44$ (d, $J=21.5 \mathrm{~Hz})$, 98.61, 78.48. HRMS (ESI) calculated $[\mathrm{M}-\mathrm{OH}]^{+}$for $\mathrm{C}_{13} \mathrm{H}_{9} \mathrm{FI}: 310.9727$, found: 310.9732 . FTIR $\left(\mathbf{c m}^{-1}\right)$ 3584, 3322, 3059, 1602, 1507, 1225, 1007, 843.

\section{[1,1'-Biphenyl]-4-yl(2-iodophenyl)methanol (3f)}<smiles>OC(c1ccc(-c2ccccc2)cc1)c1ccccc1I</smiles>

$3 f$

Following the general procedure, treatment of [1,1'-biphenyl]-4carbaldehyde $1 f \quad(0.091 \mathrm{~g}, \quad 0.5 \mathrm{mmol}), \quad$ 2-(trimethylsilyl)phenyl trifluoromethanesulfonate $2 \mathbf{a}(0.373 \mathrm{~g}, 303 \mu \mathrm{L}, 1.25 \mathrm{mmol})$ and $\mathrm{KI}(0.208$ $\mathrm{g}, 1.25 \mathrm{mmol})$ in the presence of $\mathrm{KF}(0.145 \mathrm{~g}, 2.5 \mathrm{mmol})$ and 18-crown-6 $(0.660 \mathrm{~g}, 2.5 \mathrm{mmol})$ in THF $(2.0 \mathrm{~mL})$ at $25^{\circ} \mathrm{C}$ for $24 \mathrm{~h}$ followed by quenching the reaction mixture using $4 \mathrm{~N} \mathrm{HCl}(\mathrm{HCl}$ in dioxane, $0.8 \mathrm{~mL})$, and subsequent purification via silica gel flash column chromatography $($ Pet. ether $/$ EtOAc $=90 / 10)$ of the crude reaction mixture afforded $\left[1,1^{\prime}-\right.$ biphenyl]-4-yl(2-iodophenyl)methanol 3f as a yellow oil (0.070 g, 36\% yield).

$\boldsymbol{R}_{\mathbf{f}}\left(\right.$ Pet. ether $/$ EtOAc = 85/15): 0.49; ${ }^{1} \mathbf{H}$ NMR (400 MHz, $\left.\mathbf{C D C l}_{3}\right) \delta 7.87(\mathrm{~d}, J=7.9 \mathrm{~Hz}, 1 \mathrm{H})$, 7.61-7.58 (m, 5H), 7.50-7.34 (m, 6H), 7.04-7.00 (m, 1H), $6.11(\mathrm{~s}, 1 \mathrm{H}), 2.61$ (bs, $1 \mathrm{H}) .{ }^{13} \mathbf{C}$ NMR (100 MHz, $\left.\mathbf{C D C l}_{3}\right) \delta 145.46,141.25,140.82,140.74,139.70,129.61,128.89,128.76,128.48$, 127.77, 127.46, 127.33, 127.20, 98.78, 78.92. HRMS (ESI) calculated $[\mathrm{M}-\mathrm{OH}]^{+}$for $\mathrm{C}_{13} \mathrm{H}_{9} \mathrm{INO}_{2}$ : 369.0135, found: 369.0142. FTIR (cm $\left.\mathbf{c m}^{-1}\right)$ 3357, 3057, 2363, 1486, 1005, 855.

\section{(2-Iodophenyl)(4-(trifluoromethyl)phenyl)methanol (3g)}

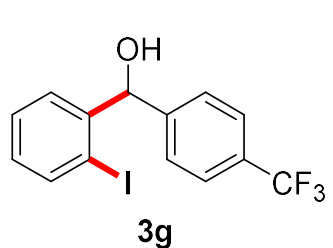

Following the general procedure, treatment of 4(trifluoromethyl)benzaldehyde $\mathbf{1 g}(0.087 \mathrm{~g}, 68 \mu \mathrm{L}, 0.5 \mathrm{mmol}), 2-$ (trimethylsilyl)phenyl trifluoromethanesulfonate $2 \mathbf{a}(0.373 \mathrm{~g}, 303 \mu \mathrm{L}, 1.25$ $\mathrm{mmol})$ and $\mathrm{KI}(0.208 \mathrm{~g}, 1.25 \mathrm{mmol})$ in the presence of KF $(0.145 \mathrm{~g}, 2.5$

mmol) and 18-crown-6 $(0.660 \mathrm{~g}, 2.5 \mathrm{mmol})$ in THF $(2.0 \mathrm{~mL})$ at $25{ }^{\circ} \mathrm{C}$ for $24 \mathrm{~h}$ followed by quenching the reaction mixture using $4 \mathrm{~N} \mathrm{HCl}(\mathrm{HCl}$ in dioxane, $0.8 \mathrm{~mL}$ ), and subsequent 
purification via silica gel flash column chromatography (Pet. ether $/$ EtOAc $=80 / 20)$ of the crude reaction mixture afforded (2-iodophenyl)(4-(trifluoromethyl)phenyl)methanol $\mathbf{3 g}$ as a yellow oil (0.115 g, 61\% yield).

$\boldsymbol{R}_{\mathbf{f}}($ Pet. ether $/$ EtOAc $=85 / 15)$ : 0.51; ${ }^{1} \mathbf{H}$ NMR (400 MHz, $\left.\mathbf{C D C l}_{3}\right) \delta$ 7.86-7.84 (m, 1H), $7.59(\mathrm{~d}$, $J=8.4 \mathrm{~Hz}, 2 \mathrm{H}), 7.53(\mathrm{~d}, J=8.2 \mathrm{~Hz}, 2 \mathrm{H}), 7.43-7.34(\mathrm{~m}, 2 \mathrm{H}), 7.03-6.99(\mathrm{~m}, 1 \mathrm{H}), 6.12(\mathrm{~s}, 1 \mathrm{H})$, 2.80 (bs, 1H). ${ }^{13} \mathbf{C}$ NMR (100 MHz, $\left.\mathbf{C D C l}_{3}\right) \delta$ 146.07, 144.94, 139.80, 129.98, 129.93 (d, $J=$ $32.5 \mathrm{~Hz}$ ), 128.96, 128.56, 127.42, 125.50 (q, $J=3.95 \mathrm{~Hz}$ ), 122.86, 98.78, 78.50. HRMS (ESI) calculated $[\mathrm{M}-\mathrm{OH}]^{+}$for $\mathrm{C}_{14} \mathrm{H}_{9} \mathrm{IN}: 317.9775$, found: 317.9781. FTIR $\left(\mathbf{c m}^{-1}\right)$ 3583, 3303, 2926, $1325,1167,1124,1066,1011$.

\section{4-(Hydroxy(2-iodophenyl)methyl)benzonitrile (3h)}<smiles>N#Cc1ccc(C(O)c2ccccc2I)cc1</smiles>

$3 \mathrm{~h}$

Following the general procedure, treatment of 4-formylbenzonitrile $\mathbf{1 h}$ (0.065g, $0.5 \mathrm{mmol}), 2$-(trimethylsilyl)phenyl trifluoromethanesulfonate 2a $(0.373 \mathrm{~g}, 303 \mu \mathrm{L}, 1.25 \mathrm{mmol})$ and $\mathrm{KI}(0.208 \mathrm{~g}, 1.25 \mathrm{mmol})$ in the presence of KF (0.145 g, $2.5 \mathrm{mmol})$ and 18-crown-6 (0.660 g, $2.5 \mathrm{mmol})$ in THF $(2.0 \mathrm{~mL})$ at $25^{\circ} \mathrm{C}$ for $24 \mathrm{~h}$ followed by quenching the reaction mixture using $4 \mathrm{~N} \mathrm{HCl}(\mathrm{HCl}$ in dioxane, $0.8 \mathrm{~mL}$ ), and subsequent purification via silica gel flash column chromatography (Pet. ether $/$ EtOAc $=80 / 20)$ of the crude reaction mixture afforded 4-(hydroxy(2iodophenyl)methyl)benzonitrile $3 \mathbf{h}$ as a yellow oil ( $0.114 \mathrm{~g}, 68 \%$ yield).

$\boldsymbol{R}_{\mathbf{f}}($ Pet. ether $/$ EtOAc $=85 / 15): 0.39 ;{ }^{1} \mathbf{H}$ NMR (400 MHz, $\left.\mathbf{C D C l}_{3}\right) \delta 7.87(\mathrm{~d}, J=7.6 \mathrm{~Hz}, 1 \mathrm{H})$, $7.60(\mathrm{~d}, J=8.7 \mathrm{~Hz}, 2 \mathrm{H}), 7.54$ (d, $J=8.3 \mathrm{~Hz}, 2 \mathrm{H}), 7.44-7.35$ (m, 2H), 7.04-6.99 (m, 1H), 6.13 (s, 1H), 2.65 (bs, 1H). ${ }^{13} \mathbf{C}$ NMR (100 MHz, CDCl $) \delta 147.35,144.61,139.77,132.29,130.08$, 128.97, 128.53, 127.64, 118.79, 111.41 98.63, 78.30. HRMS (ESI) calculated $[\mathrm{M}-\mathrm{OH}]^{+}$for $\mathrm{C}_{14} \mathrm{H}_{9} \mathrm{IN}: 317.9774$, found: 317.9781. FTIR ( $\left.\mathbf{c m}^{-1}\right)$ 3584, 3323, 2364, 1569, 1454, 1241, 1008 , 886.

\section{Methyl-4-(hydroxy(2-iodophenyl)methyl)benzoate (3i)}<smiles>CC(=O)c1ccc(C(O)c2ccccc2I)cc1</smiles>

$3 \mathbf{i}$

Following the general procedure, treatment of methyl 4formylbenzoate 1i $(0.082 \mathrm{~g}, 0.5 \mathrm{mmol})$, 2-(trimethylsilyl)phenyl trifluoromethanesulfonate $2 \mathrm{a}(0.373 \mathrm{~g}, 303 \mu \mathrm{L}, 1.25 \mathrm{mmol})$ and $\mathrm{KI}$ $(0.208 \mathrm{~g}, 1.25 \mathrm{mmol})$ in the presence of KF $(0.145 \mathrm{~g}, 2.5 \mathrm{mmol})$ and 
18-crown-6 $(0.660 \mathrm{~g}, 2.5 \mathrm{mmol})$ in THF $(2.0 \mathrm{~mL})$ at $25{ }^{\circ} \mathrm{C}$ for $24 \mathrm{~h}$ followed by quenching the reaction mixture using $4 \mathrm{~N} \mathrm{HCl}(\mathrm{HCl}$ in dioxane, $0.8 \mathrm{~mL})$, and subsequent purification via silica gel flash column chromatography (Pet. ether $/$ EtOAc $=90 / 10$ ) of the crude reaction mixture afforded methyl-4-(hydroxy(2-iodophenyl)methyl)benzoate $\mathbf{3 i}$ as a yellow oil $(0.126 \mathrm{~g}, 68 \%$ yield).

$\boldsymbol{R}_{\mathbf{f}}($ Pet. ether $/$ EtOAc $=85 / 15): 0.41 ;{ }^{1} \mathbf{H}$ NMR (400 MHz, $\left.\mathbf{C D C l}_{3}\right) \delta$ 7.98-7.96 (m, 2H), $8.83(\mathrm{~d}$, $J=7.9 \mathrm{~Hz}, 1 \mathrm{H}), 7.47(\mathrm{~d}, J=8.1 \mathrm{~Hz}, 2 \mathrm{H}), 7.42\left(\mathrm{dd}, J_{l}=7.8 \mathrm{~Hz}, J_{2}=1.5 \mathrm{~Hz}, 1 \mathrm{H}\right), 7.34(\mathrm{t}, J=7.5$ $\mathrm{Hz}, 1 \mathrm{H}), 7.00-6.96(\mathrm{~m}, 1 \mathrm{H}), 6.10(\mathrm{~s}, 1 \mathrm{H}), 3.88$ (s, 3H), 2.86 (bs, 1H). ${ }^{13} \mathbf{C}$ NMR (100 MHz, $\left.\mathbf{C D C l}_{3}\right) \delta 167.06,147.36,145.12,139.72,129.85,129.83,129.45,128.85,128.63,127.11$, 98.80, 78.65, 52.26. HRMS (ESI) calculated $[\mathrm{M}-\mathrm{OH}]{ }^{+}$for $\mathrm{C}_{15} \mathrm{H}_{12} \mathrm{INO}_{2}$ : 350.9876, found: 350.9881. FTIR (cm $\left.{ }^{-1}\right)$ 3477, 2363, 1718, 1434, 1282, 1110, 1010, 870.

\section{4-(Hydroxy(2-iodophenyl)methyl)benzaldehyde (3j)}

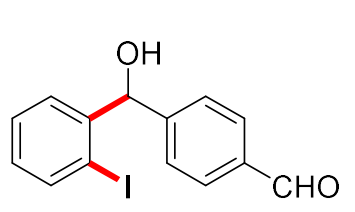

3j

Following the general procedure, treatment of terephthalaldehyde $\mathbf{1 j}$ $(0.034 \mathrm{~g}, 0.25 \mathrm{mmol}), 2$-(trimethylsilyl)phenyl trifluoromethanesulfonate 2a $(0.186 \mathrm{~g}, 151 \mu \mathrm{L}, 0.625 \mathrm{mmol})$ and $\mathrm{KI}(0.104 \mathrm{~g}, 0.625 \mathrm{mmol})$ in the presence of KF $(0.073 \mathrm{~g}, 1.25 \mathrm{mmol})$ and 18-crown-6 (0.330 g, 1.25

$\mathrm{mmol})$ in THF $(1.0 \mathrm{~mL})$ at $25^{\circ} \mathrm{C}$ for $24 \mathrm{~h}$ followed by quenching the reaction mixture using $4 \mathrm{~N}$ $\mathrm{HCl}(\mathrm{HCl}$ in dioxane, $0.4 \mathrm{~mL}$ ), and subsequent purification via silica gel flash column chromatography (Pet. ether $/$ EtOAc $=80 / 20$ ) of the crude reaction mixture afforded 4(hydroxy(2-iodophenyl)methyl)benzaldehyde $\mathbf{3 j}$ as a colourless oil $(0.050 \mathrm{~g}, 59 \%$ yield).

$\boldsymbol{R}_{\mathbf{f}}($ Pet. ether $/$ EtOAc $=85 / 15)$ : 0.41; ${ }^{1} \mathbf{H}$ NMR (400 MHz, $\left.\mathbf{C D C l}_{3}\right) \delta 9.97(\mathrm{~s}, 1 \mathrm{H}), 7.85-7.83(\mathrm{~m}$, $3 \mathrm{H}), 7.59(\mathrm{~d}, J=8.4 \mathrm{~Hz}, 2 \mathrm{H}), 7.42\left(\mathrm{dd}, J_{l}=7.7 \mathrm{~Hz}, J_{2}=1.6 \mathrm{~Hz}, 1 \mathrm{H}\right), 7.36(\mathrm{t}, J=7.3 \mathrm{~Hz}, 1 \mathrm{H})$, 7.03-6.99 (m, 1H),6.15 (s, 1H), 2.76 (bs, 1H). ${ }^{13}$ C NMR (100 MHz, CDCl $) \delta$ 192.16, 149.03, 144.97, 139.82, 136.77, 130.05, 130.02, 128.99, 128.68, 127.69, 98.81, 78.67. HRMS (ESI) calculated $[\mathrm{M}-\mathrm{OH}]^{+}$for $\mathrm{C}_{14} \mathrm{H}_{10} \mathrm{IO}: 320.9771$, found: 320.9774. FTIR (cm $\left.{ }^{-1}\right)$ 3747, 3584, 2964, 2364, 1740, 1595, 1425, 1219.

\section{(3-Bromophenyl)(2-iodophenyl)methanol (3k) ${ }^{4}$}

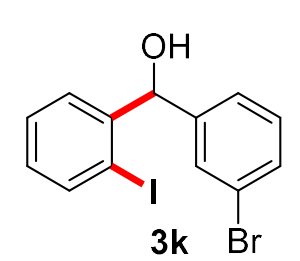

Following the general procedure, treatment of 3-bromobenzaldehyde $\mathbf{1 k}$ (0.093 g, $0.059 \mu \mathrm{L}, 0.5 \mathrm{mmol}), 2$-(trimethylsilyl)phenyl trifluoromethane 
sulfonate $2 \mathrm{a}(0.373 \mathrm{~g}, 303 \mu \mathrm{L}, 1.25 \mathrm{mmol})$ and $\mathrm{KI}(0.208 \mathrm{~g}, 1.25 \mathrm{mmol})$ in the presence of $\mathrm{KF}$ $(0.145 \mathrm{~g}, 2.5 \mathrm{mmol})$ and 18-crown-6 $(0.660 \mathrm{~g}, 2.5 \mathrm{mmol})$ in THF $(2.0 \mathrm{~mL})$ at $25{ }^{\circ} \mathrm{C}$ for $24 \mathrm{~h}$ followed by quenching the reaction mixture using $4 \mathrm{~N} \mathrm{HCl}(\mathrm{HCl}$ in dioxane, $0.8 \mathrm{~mL})$, and subsequent purification viasilica gel flash column chromatography (Pet. ether $/$ EtOAc $=90 / 10$ ) of the crude reaction mixture afforded (3-bromophenyl)(2-iodophenyl)methanol $\mathbf{3 k}$ as a yellow oil ( $0.130 \mathrm{~g}, 66 \%$ yield).

$\boldsymbol{R}_{\mathbf{f}}($ Pet. ether $/$ EtOAc $=85 / 15): 0.53 ;{ }^{1} \mathbf{H}$ NMR (400 $\left.\mathbf{M H z}, \mathbf{C D C l}_{3}\right) \delta 7.84\left(\mathrm{dd}, J_{1}=7.9 \mathrm{~Hz}, J_{2}\right.$ $=0.8 \mathrm{~Hz}, 1 \mathrm{H}), 7.58(\mathrm{~s}, 1 \mathrm{H}), 7.45-7.35(\mathrm{~m}, 3 \mathrm{H}), 7.31(\mathrm{~d}, J=7.8 \mathrm{~Hz}, 1 \mathrm{H}), 7.19(\mathrm{t}, J=7.8 \mathrm{~Hz}, 1 \mathrm{H})$, $7.00\left(\mathrm{td}, J_{1}=7.7, J_{2}=1.7 \mathrm{~Hz}, 1 \mathrm{H}\right), 6.02(\mathrm{~d}, J=2.6 \mathrm{~Hz}, 1 \mathrm{H}), 2.63(\mathrm{~d}, J=3.4 \mathrm{~Hz}, 1 \mathrm{H}) .{ }^{13} \mathbf{C} \mathbf{~ N M R}$ (100 MHz, CDCl $) \delta 144.91,144.49,139.75,130.94,130.21,130.12,129.87,128.89,128.51$, 125.91, 122.74, 98.76, 78.43. HRMS (ESI) calculated $[\mathrm{M}-\mathrm{OH}]^{+}$for $\mathrm{C}_{13} \mathrm{H}_{9} \mathrm{BrI}$ : 370.8927, found: 370.8933. Isotopic Mass: $[\mathrm{M}-\mathrm{OH}+2]^{+}$372.89143. FTIR (cm-1) 3579, 3341, 2916, 1566, 1428, 1007.

\section{(2-Fluorophenyl)(2-iodophenyl)methanol (3I)}

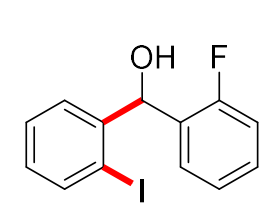

3)

Following the general procedure, treatment of 2-fluorobenzaldehyde $11(0.062 \mathrm{~g}$, $52.85 \mu \mathrm{L}, 0.5 \mathrm{mmol}$ ), 2-(trimethylsilyl)phenyl trifluoromethanesulfonate $\mathbf{2 a}$

$(0.373 \mathrm{~g}, 303 \mu \mathrm{L}, 1.25 \mathrm{mmol})$ and $\mathrm{KI}(0.208 \mathrm{~g}, 1.25 \mathrm{mmol})$ in the presence of $\mathrm{KF}(0.145 \mathrm{~g}, 2.5 \mathrm{mmol})$ and 18-crown-6 (0.660 g, $2.5 \mathrm{mmol})$ in THF (2.0 mL) at $25{ }^{\circ} \mathrm{C}$ for $24 \mathrm{~h}$ followed by quenching the reaction mixture using $4 \mathrm{~N} \mathrm{HCl}(\mathrm{HCl}$ in dioxane, 0.8 $\mathrm{mL}$ ), and subsequent purification via silica gel flash column chromatography (Pet. ether /EtOAc $=90 / 10)$ of the crude reaction mixture afforded (2-fluorophenyl)(2-iodophenyl)methanol $\mathbf{3 l}$ as a yellow oil (0.059 g, 36\% yield).

$\boldsymbol{R}_{\mathbf{f}}($ Pet. ether $/$ EtOAc $=85 / 15): 0.56 ;{ }^{1} \mathbf{H}$ NMR (400 $\left.\mathbf{M H z}, \mathbf{C D C l}_{3}\right) \delta 7.85\left(\mathrm{dd}, J_{1}=7.9 \mathrm{~Hz}, J_{2}=\right.$ $1.2 \mathrm{~Hz}, 1 \mathrm{H}), 7.49$ (dd, $\left.J_{l}=7.9 \mathrm{~Hz}, J_{2}=1.7 \mathrm{~Hz}, 1 \mathrm{H}\right), 7.40-7.36(\mathrm{~m}, 1 \mathrm{H}), 7.33-7.27(\mathrm{~m}, 1 \mathrm{H}), 7.25-$ $7.21(\mathrm{~m}, 1 \mathrm{H}), 7.13-7.00(\mathrm{~m}, 3 \mathrm{H}), 6.29(\mathrm{~d}, J=3.9 \mathrm{~Hz}, 1 \mathrm{H}), 2.59(\mathrm{~d}, J=4.3 \mathrm{~Hz}, 1 \mathrm{H}) .{ }^{13} \mathbf{C} \mathbf{~ N M R}$ $\left(\mathbf{1 0 0} \mathbf{M H z}, \mathbf{C D C l}_{3}\right) \delta 160.74(\mathrm{~d}, J=217.8 \mathrm{~Hz}), 140.00,139.71,129.82,129.74,129.18(\mathrm{~d}, J=$ $13.2 \mathrm{~Hz}), 128.65$ (d, $J=3.8 \mathrm{~Hz}), 128.55,128.37,124.33$ (d, $J=3.3 \mathrm{~Hz}), 115.56$ (d, $J=22.1 \mathrm{~Hz})$, 98.73, 73.63 (d, $J=3.7 \mathrm{~Hz}$ ). HRMS (ESI) calculated $[\mathrm{M}-\mathrm{OH}]^{+}$for $\mathrm{C}_{13} \mathrm{H}_{9} \mathrm{FI}: 310.9727$, found: 310.9730. FTIR (cm-1) 3584, 3345, 3058, 2363, 1488, 1456, 1227, 1008. 


\section{(2-Chlorophenyl)(2-iodophenyl)methanol (3m) ${ }^{4}$}

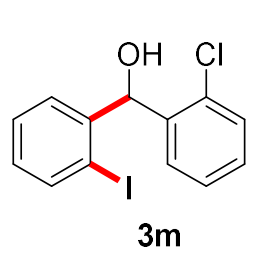

Following the general procedure, treatment of 2-chlorobenzaldehyde $\mathbf{1 m}$ $(0.070 \mathrm{~g}, \quad 56.30 \mu \mathrm{L}, \quad 0.5 \mathrm{mmol}), \quad 2$-(trimethylsilyl)phenyl trifluoromethane sulfonate $2 \mathrm{a}(0.373 \mathrm{~g}, 303 \mu \mathrm{L}, 1.25 \mathrm{mmol})$ and $\mathrm{KI}(0.208 \mathrm{~g}, 1.25 \mathrm{mmol})$ in the presence of KF (0.145 g, $2.5 \mathrm{mmol})$ and 18-crown-6 (0.660 g, $2.5 \mathrm{mmol})$ in THF $(2.0 \mathrm{~mL})$ at $25^{\circ} \mathrm{C}$ for $24 \mathrm{~h}$ followed by quenching the reaction mixture using $4 \mathrm{~N} \mathrm{HCl}(\mathrm{HCl}$ in dioxane, $0.8 \mathrm{~mL}$ ), and subsequent purification via silica gel flash column chromatography (Pet. ether $/$ EtOAc $=90 / 10)$ of the crude reaction mixture afforded(2-chlorophenyl)(2iodophenyl)methanol $\mathbf{3 m}$ as a yellowoil ( $0.065 \mathrm{~g}, 38 \%$ yield).

$\boldsymbol{R}_{\mathbf{f}}($ Pet. ether $/$ EtOAc $=85 / 15): 0.58 ;{ }^{1} \mathbf{H}$ NMR (400 MHz, $\left.\mathbf{C D C l}_{3}\right) \delta 7.87\left(\mathrm{dd}, J_{1}=7.8 \mathrm{~Hz}, J_{2}=\right.$ $0.9 \mathrm{~Hz}, 1 \mathrm{H}), 7.41-7.25(\mathrm{~m}, 6 \mathrm{H}), 7.04-7.00(\mathrm{~m}, 1 \mathrm{H}), 6.30(\mathrm{~d}, J=3.7 \mathrm{~Hz}, 1 \mathrm{H}), 2.58(\mathrm{~d}, J=4.0 \mathrm{~Hz}$, 1H). ${ }^{13} \mathbf{C}$ NMR (100 MHz, $\left.\mathbf{C D C l}_{3}\right) \delta 143.88,139.79,139.52,133.87,129.77,129.25,128.56$, 128.48, 127.11, 99.72, 76.49. HRMS (ESI) calculated [M-OH] ${ }^{+}$for $\mathrm{C}_{13} \mathrm{H}_{9} \mathrm{ClI}$ : 326.9432, found: 326.9436. Isotopic Mass: [M-OH+2] 328.9406. FTIR (cm-1) 3583, 3301, 3059, 1626, 1468, $1436,1055,1009$.

\section{(2-Iodophenyl)(2-nitrophenyl)methanol (3n)}<smiles>O=[N+]([O-])c1ccccc1C(O)c1ccccc1[Hg]c1ccccc1</smiles>

Following the general procedure, treatment of 2-nitrobenzaldehyde 1n $(0.076$ g, $0.5 \mathrm{mmol}), 2$-(trimethylsilyl)phenyl trifluoromethanesulfonate $\mathbf{2 a}(0.373 \mathrm{~g}$, $303 \mu \mathrm{L}, 1.25 \mathrm{mmol})$ and $\mathrm{KI}(0.208 \mathrm{~g}, 1.25 \mathrm{mmol})$ in the presence of $\mathrm{KF}(0.145$ $\mathrm{g}, 2.5 \mathrm{mmol})$ and 18 -crown-6 $(0.660 \mathrm{~g}, 2.5 \mathrm{mmol})$ in THF $(2.0 \mathrm{~mL})$ at $25{ }^{\circ} \mathrm{C}$ for $24 \mathrm{~h}$ followed by quenching the reaction mixture using $4 \mathrm{~N} \mathrm{HCl}(\mathrm{HCl}$ in dioxane, $0.8 \mathrm{~mL})$, and subsequent purification via silica gel flash column chromatography (Pet. ether /EtOAc $=$ 90/10) of the crude reaction mixture afforded (2-iodophenyl)(2-nitrophenyl)methanol $\mathbf{3 n}$ as a yellow oil ( $0.098 \mathrm{~g}, 55 \%$ yield $)$.

$\boldsymbol{R}_{\mathbf{f}}($ Pet. ether $/$ EtOAc $=85 / 15): 0.35 ;{ }^{1} \mathbf{H}$ NMR (400 MHz, $\left.\mathbf{C D C l}_{3}\right) \delta 7.99\left(\mathrm{dd}, J_{l}=8.1 \mathrm{~Hz}\right.$, $\left.J_{2}=1.2 \mathrm{~Hz}, 1 \mathrm{H}\right), 7.83-7.81(\mathrm{~m}, 1 \mathrm{H}), 7.56\left(\mathrm{td}, J_{1}=7.7 \mathrm{~Hz}, J_{2}=1.2 \mathrm{~Hz}, 1 \mathrm{H}\right), 7.51-7.45(\mathrm{~m}, 2 \mathrm{H})$, $7.39(\mathrm{t}, J=7.5 \mathrm{~Hz}, 1 \mathrm{H}), 7.32-7.30(\mathrm{~m}, 1 \mathrm{H}), 7.03\left(\mathrm{td}, J_{1}=7.7, J_{2 .}=1.7 \mathrm{~Hz}, 1 \mathrm{H}\right), 6.43(\mathrm{~d}, J=3.7$ $\mathrm{Hz}, 1 \mathrm{H}), 3.31(\mathrm{~d}, J=4.0 \mathrm{~Hz}, 1 \mathrm{H}) .{ }^{13} \mathbf{C}$ NMR (100 MHz, CDCl$) \delta 149.50,143.18,139.76$, $137.17,133.74,129.86,129.78,128.91,128.56,128.15,124.83,99.26,74.78$. HRMS (ESI) 
calculated $[\mathrm{M}-\mathrm{OH}]^{+}$for $\mathrm{C}_{13} \mathrm{H}_{9} \mathrm{INO}_{2}: 337.9672$, found: 337.9681. FTIR (cm $\left.{ }^{-1}\right)$ 3402, 2924, 1643, $1524,1345,1012$.

\section{(3,4-Dichlorophenyl)(2-iodophenyl)methanol (3o)}<smiles>OC(c1ccc(Cl)c(Cl)c1)c1ccccc1I</smiles>

30

Following the general procedure, treatment of 3,4-dichlorobenzaldehyde 10 (0.0875g, $0.5 \mathrm{mmol}), 2$-(trimethylsilyl)phenyl trifluoromethanesulfonate 2a $(0.373 \mathrm{~g}, 303 \mu \mathrm{L}, 1.25 \mathrm{mmol})$ and $\mathrm{KI}(0.208 \mathrm{~g}, 1.25 \mathrm{mmol})$ in the presence of KF (0.145 g, $2.5 \mathrm{mmol})$ and 18-crown-6 (0.660 g, $2.5 \mathrm{mmol})$ in THF (2.0 $\mathrm{mL})$ at $25{ }^{\circ} \mathrm{C}$ for $24 \mathrm{~h}$ followed by quenching the reaction mixture using $4 \mathrm{~N} \mathrm{HCl}(\mathrm{HCl}$ in dioxane, $0.8 \mathrm{~mL}$ ), and subsequent purification via silica gel flash column chromatography (Pet. ether $/$ EtOAc $=88 / 12)$ of the crude reaction mixture afforded (3,4-dichlorophenyl)(2iodophenyl)methanol 30 as a yellow oil $(0.123 \mathrm{~g}, 65 \%$ yield $)$.

$\boldsymbol{R}_{\mathbf{f}}($ Pet. ether $/$ EtOAc $=85 / 15): 0.49 ;{ }^{1} \mathbf{H}$ NMR (400 MHz, $\left.\mathbf{C D C l}_{3}\right) \delta 7.83\left(\mathrm{dd}, J_{l}=7.8 \mathrm{~Hz}, J_{2}=\right.$ $0.7 \mathrm{~Hz}, 1 \mathrm{H}), 7.50(\mathrm{~d}, J=1.9 \mathrm{~Hz}, 1 \mathrm{H}), 7.40-7.34$ (m, 3H), 7.20-7.17 (m, 1H), 7.03-6.98 (m, 1H)5.98 (d, $J=3.0 \mathrm{~Hz}, 1 \mathrm{H}), 2.89(\mathrm{~d}, J=3.5 \mathrm{~Hz}, 1 \mathrm{H}) .{ }^{13} \mathbf{C} \mathbf{N M R}\left(\mathbf{1 0 0} \mathbf{M H z}, \mathbf{C D C l}_{3}\right) \delta 144.55$, 142.36, 139.79, 132.66, 131.79, 130.47, 130.02, 129.11, 128.96, 128.36, 126.57, 98.66, 77.94 . HRMS (ESI) calculated [M-OH] ${ }^{+}$for $\mathrm{C} 13 \mathrm{H} 8 \mathrm{Cl}_{2} \mathrm{I}$ : 360.9042, found: 360.9048. Isotopic Mass: $[\mathrm{M}-\mathrm{OH}+2]^{+}$362.9019, [M-OH+4] $]^{+}$364.8993. FTIR (cm $\left.{ }^{-1}\right)$ 3583, 3298, 3058, 2364, 1466, 1390, $1032,889$.

\section{(2-Chloro-6-fluorophenyl)(2-iodophenyl)methanol (3p)}

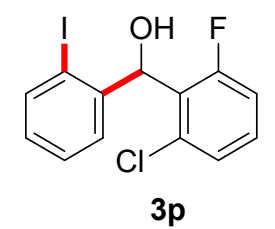

Following the general procedure, treatment of 2-chloro-6-fluorobenzaldehyde $1 \mathbf{p}(0.0792 \mathrm{~g}, 0.5 \mathrm{mmol}), 2$-(trimethylsilyl)phenyl trifluoromethanesulfonate $\mathbf{2 a}$ $(0.373 \mathrm{~g}, 303 \mu \mathrm{L}, 1.25 \mathrm{mmol})$ and $\mathrm{KI}(0.208 \mathrm{~g}, 1.25 \mathrm{mmol})$ in the presence of $\mathrm{KF}(0.145 \mathrm{~g}, 2.5 \mathrm{mmol})$ and 18 -crown-6 $(0.660 \mathrm{~g}, 2.5 \mathrm{mmol})$ in THF $(2.0 \mathrm{~mL})$ at $25{ }^{\circ} \mathrm{C}$ for $24 \mathrm{~h}$ followed by quenching the reaction mixture using $4 \mathrm{~N} \mathrm{HCl}(\mathrm{HCl}$ in dioxane, 0.8 $\mathrm{mL}$ ), and subsequent purification via silica gel flash column chromatography (Pet. ether /EtOAc $=90 / 10)$ of the crude reaction mixture afforded (2-chloro-6-fluorophenyl)(2iodophenyl)methanol $\mathbf{3 p}$ as a white solid $(0.110 \mathrm{~g}, 61 \%$ yield $)$.

$\boldsymbol{R}_{\mathbf{f}}($ Pet. ether $/$ EtOAc $=85 / 15): 0.61 ;{ }^{1} \mathbf{H}$ NMR $\left(400 \mathbf{M H z}, \mathbf{C D C l}_{3}\right) \delta 7.87\left(\mathrm{dd}, J_{l}=8.0 \mathrm{~Hz}, J_{2}=\right.$ $1.1 \mathrm{~Hz}, 1 \mathrm{H}), 7.52(\mathrm{~d}, J=7.7 \mathrm{~Hz}, 1 \mathrm{H}), 7.38-7.34(\mathrm{~m}, 1 \mathrm{H}), 7.28-7.22(\mathrm{~m}, 2 \mathrm{H}), 7.03-6.96(\mathrm{~m}, 2 \mathrm{H})$, 
$6.32(\mathrm{~d}, J=6.4 \mathrm{~Hz}, 1 \mathrm{H}), 2.80(\mathrm{~d}, J=4.2 \mathrm{~Hz}, 1 \mathrm{H}) .{ }^{13} \mathbf{C} \mathbf{N M R}\left(\mathbf{1 0 0} \mathbf{M H z}, \mathbf{C D C l}_{3}\right) \delta 161.785(\mathrm{~d}, J$ $=251.8 \mathrm{~Hz}), 143.13(\mathrm{~d}, J=1.7 \mathrm{~Hz}), 139.88,136.39(\mathrm{~d}, J=6.5 \mathrm{~Hz}), 130.05(\mathrm{~d}, J=10.0 \mathrm{~Hz})$, 129.55, 128.26 (d, $J=3.9 \mathrm{~Hz}), 128.07,127.00$ (d, $J=13.8 \mathrm{~Hz}), 125.91$ (d, $J=3.6 \mathrm{~Hz}), 115.35$ $(\mathrm{d}, J=23.4 \mathrm{~Hz}), 97.98,74.88$. HRMS (ESI) calculated $[\mathrm{M}-\mathrm{OH}]^{+}$for $\mathrm{C}_{13} \mathrm{H}_{8} \mathrm{ClFI}: 344.9338$, found: 344.9343. Isotopic Mass: $[\mathrm{M}-\mathrm{OH}+2]^{+}$346.9315. FTIR (cm-1) 3747, 3584, 2925, 2364, $1458,1170,1014,902$.

\section{(3,5-Bis(trifluoromethyl)phenyl)(2-iodophenyl)methanol (3q)}<smiles>OC(c1cc(C(F)(F)F)cc(C(F)(F)F)c1)c1ccccc1I</smiles>

$3 q$

Following the general procedure, treatment of 3,5bis(trifluoromethyl)benzaldehyde 1q $(0.121 \mathrm{~g}, 82 \mu \mathrm{L} 0.5 \mathrm{mmol}), 2$ (trimethylsilyl)phenyl trifluoromethanesulfonate 2a $(0.373 \mathrm{~g}, 303 \mu \mathrm{L}, 1.25$ mmol) and $\mathrm{KI}(0.208 \mathrm{~g}, 1.25 \mathrm{mmol})$ in the presence of KF $(0.145 \mathrm{~g}, 2.5$ mmol) and 18-crown-6 (0.660 g, $2.5 \mathrm{mmol})$ in THF $(2.0 \mathrm{~mL})$ at $25^{\circ} \mathrm{C}$ for $24 \mathrm{~h}$ followed by quenching the reaction mixture using $4 \mathrm{~N} \mathrm{HCl}(\mathrm{HCl}$ in dioxane, $0.8 \mathrm{~mL})$, and subsequent purification via silica gel flash column chromatography (Pet. ether $/$ EtOAc $=90 / 10$ ) of the crude reaction mixture afforded (3,5-bis(trifluoromethyl)phenyl)(2-iodophenyl)methanol $3 q$ as a yellow oil $(0.133 \mathrm{~g}, 60 \%$ yield $)$.

$\boldsymbol{R}_{\mathbf{f}}($ Pet. ether $/$ EtOAc $=85 / 15)$ : 0.56; ${ }^{1} \mathbf{H}$ NMR (400 MHz, $\left.\mathbf{C D C l}_{3}\right) \delta 7.91(\mathrm{~s}, 2 \mathrm{H}), 7.88(\mathrm{~d}, J=$ 8.1 Hz, 1H), $7.81(\mathrm{~s}, 1 \mathrm{H}), 7.41-7.35(\mathrm{~m}, 2 \mathrm{H}), 7.07-7.02(\mathrm{~m}, 1 \mathrm{H}), 6.20(\mathrm{~s}, 1 \mathrm{H}), 2.80(\mathrm{bs}, 1 \mathrm{H}) .{ }^{13} \mathbf{C}$ NMR (100 MHz, $\left.\mathbf{C D C l}_{3}\right) \delta$ 143.83, 143.35, 139.06, $130.82(\mathrm{q}, J=33 \mathrm{~Hz}), 129.46,128.29$, 127.55, 126.23, $122.41(\mathrm{q}, J=272 \mathrm{~Hz}), 120.79(\mathrm{p}, J=3.8 \mathrm{~Hz}), 97.75,77.06$. HRMS (ESI) calculated $[\mathrm{M}-\mathrm{OH}]^{+}$for $\mathrm{C}_{15} \mathrm{H}_{8} \mathrm{IF}_{6} \mathrm{I}:$ 428.9569, found: 428.9573. FTIR (cm $\left.{ }^{-1}\right)$ 3319, 1374, 1280 , $1173,1134,900$.

\section{(2-Iodophenyl)(naphthalen-2-yl)methanol (3r)}<smiles>OC(c1ccc2ccccc2c1)c1ccccc1Br</smiles>

Following the general procedure, treatment of 2-naphthaldehyde $\mathbf{1 r}$ (0.078 g, $0.5 \mathrm{mmol})$, 2-(trimethylsilyl)phenyl trifluoromethanesulfonate 2a $(0.373 \mathrm{~g}, 303 \mu \mathrm{L}, 1.25 \mathrm{mmol})$ and $\mathrm{KI}(0.208 \mathrm{~g}, 1.25 \mathrm{mmol})$ in the presence of KF $(0.145 \mathrm{~g}, 2.5 \mathrm{mmol})$ and 18-crown-6 (0.660 g, 2.5 mmol) in THF $(2.0 \mathrm{~mL})$ at $25^{\circ} \mathrm{C}$ for $24 \mathrm{~h}$ followed by quenching the reaction mixture using $4 \mathrm{~N}$ $\mathrm{HCl}(\mathrm{HCl}$ in dioxane, $0.8 \mathrm{~mL}$ ), and subsequent purification via silica gel flash column 
chromatography (Pet. ether $/$ EtOAc $=90 / 10)$ of the crude reaction mixture afforded (2iodophenyl)(naphthalen-2-yl)methanol 3r as a yellow oil (0.056 g, 31\% yield).

$\boldsymbol{R}_{\mathbf{f}}($ Pet. ether $/$ EtOAc $=85 / 15): 0.43 ;{ }^{1} \mathbf{H}$ NMR (400 MHz, $\left.\mathbf{C D C l}_{3}\right) \delta 7.91(\mathrm{~s}, 1 \mathrm{H}), 7.87-7.80(\mathrm{~m}$, $4 \mathrm{H}), 7.53\left(\mathrm{dd}, J_{1}=7.8 \mathrm{~Hz}, J_{2}=1.5 \mathrm{~Hz}, 1 \mathrm{H}\right), 7.51-7.48(\mathrm{~m}, 2 \mathrm{H}), 7.47-7.46(\mathrm{~m}, 1 \mathrm{H}), 7.39-7.35(\mathrm{~m}$, $1 \mathrm{H}), 7.01\left(\mathrm{td}, J_{l}=7.7 \mathrm{~Hz}, J_{2}=1.7 \mathrm{~Hz}, 1 \mathrm{H}\right), 6.23(\mathrm{~s}, 1 \mathrm{H}), 2.56(\mathrm{bs}, 1 \mathrm{H}) .{ }^{13} \mathbf{C}$ NMR (100 MHz, $\left.\mathbf{C D C l}_{3}\right) \delta 145.38,139.74,139.62,133.34,133.04,129.69,128.76,128.73,128.41,128.31$, 127.80, 126.32, 126.23, 126.08, 125.29, 99.04, 79.26. HRMS (ESI) calculated $[\mathrm{M}-\mathrm{OH}]^{+}$for $\mathrm{C}_{17} \mathrm{H}_{12} \mathrm{I}$ : 342.9978, found: 342.9984. FTIR (cm-1) 3340, 3055, 1562, 1432, 1007, 859.

\section{(2-Iodophenyl)(pyren-1-yl)methanol (3s)}<smiles>OC(c1ccccc1I)c1ccc2ccc3cccc4ccc1c2c34</smiles>

3s

Following the general procedure, treatment of pyrene-1-carbaldehyde 1s (0.115 g, $0.5 \mathrm{mmol}), 2$-(trimethylsilyl)phenyl trifluoromethanesulfonate 2a $(0.373 \mathrm{~g}, 303 \mu \mathrm{L}, 1.25 \mathrm{mmol})$ and $\mathrm{KI}(0.208 \mathrm{~g}, 1.25 \mathrm{mmol})$ in the presence of KF (0.145 g, $2.5 \mathrm{mmol})$ and 18-crown-6 (0.660 g, $2.5 \mathrm{mmol})$ in THF $(2.0 \mathrm{~mL})$ at $25^{\circ} \mathrm{C}$ for $24 \mathrm{~h}$ followed by quenching the reaction mixture using $4 \mathrm{~N} \mathrm{HCl}$ ( $\mathrm{HCl}$ in dioxane, $0.8 \mathrm{~mL}$ ), and subsequent purification via silica gel flash column chromatography (Pet. ether $/$ EtOAc $=90 / 10$ ) of the crude reaction mixture afforded (2iodophenyl)(pyren-1-yl)methanol 3s as a brown oil (0.06 g, 28\% yield).

$\boldsymbol{R}_{\mathbf{f}}$ (Pet. ether $/$ EtOAc $\left.=85 / 15\right): 0.43 ;{ }^{1} \mathbf{H}$ NMR (400 $\left.\mathbf{M H z}, \mathbf{C D C l}_{3}\right) \delta$ 8.19-7.92 (m, 9H), $7.88(\mathrm{~d}$, $J=8.9 \mathrm{~Hz}, 1 \mathrm{H}), 7.31-7.23(\mathrm{~m}, 2 \mathrm{H}), 6.98\left(\mathrm{td}, J_{1}=7.6 \mathrm{~Hz}, J_{2}=1.8 \mathrm{~Hz}, 1 \mathrm{H}\right), 6.93(\mathrm{~s}, 1 \mathrm{H}), 2.73(\mathrm{~d}, J$ $=2.8 \mathrm{~Hz}, 1 \mathrm{H}) .{ }^{13} \mathbf{C}$ NMR (100 MHz, $\left.\mathbf{C D C l} 3\right) \delta 145.29,139.84,135.05,131.39,131.22,130.74$, $129.73,129.14,128.66,128.23,127.67,127.57,126.14,125.54,125.44,125.15,125.05,124.92$, 124.82, 124.51, 123.10, 99.68, 76.72. HRMS (ESI) calculated $[\mathrm{M}-\mathrm{OH}]^{+}$for $\mathrm{C}_{23} \mathrm{H}_{14} \mathrm{I}: 417.0135$, found: 417.0144. FTIR ( $\left.\mathbf{c m}^{-1}\right)$ 3742, 3585, 3276, 3043, 2363, 1428, 1008, 842.

\section{(2-Iodophenyl)(thiophen-2-yl)methanol (3t)}

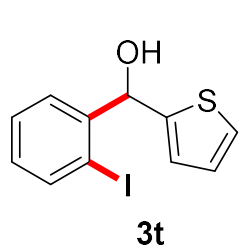

Following the general procedure, treatment of thiophene-2-carbaldehyde 1t $(0.056 \mathrm{~g}, 47 \mu \mathrm{L}, 0.5 \mathrm{mmol}), 2$-(trimethylsilyl)phenyl trifluoromethanesulfonate 2a $(0.373 \mathrm{~g}, 303 \mu \mathrm{L}, 1.25 \mathrm{mmol})$ and $\mathrm{KI}(0.208 \mathrm{~g}, 1.25 \mathrm{mmol})$ in the presence of $\mathrm{KF}(0.145 \mathrm{~g}, 2.5 \mathrm{mmol})$ and 18-crown-6 (0.660 g, $2.5 \mathrm{mmol})$ in THF (2.0 mL) at $25{ }^{\circ} \mathrm{C}$ for $24 \mathrm{~h}$ followed by quenching the reaction mixture using $4 \mathrm{~N} \mathrm{HCl}(\mathrm{HCl}$ in dioxane, 0.8 
$\mathrm{mL}$ ), and subsequent purification via silica gel flash column chromatography (Pet. ether /EtOAc $=80 / 20)$ of the crude reaction mixture afforded (2-iodophenyl)(thiophen-2-yl)methanol 3t as a yellow oil (0.038 g, 24\% yield).

$\boldsymbol{R}_{\mathbf{f}}($ Pet. ether $/$ EtOAc $=85 / 15): 0.37 ;{ }^{1} \mathbf{H}$ NMR (400 $\left.\mathbf{M H z}, \mathbf{C D C l}_{3}\right) \delta 7.83\left(\mathrm{dd}, J_{1}=7.8 \mathrm{~Hz}, J_{2}=\right.$ $1.0 \mathrm{~Hz}, 1 \mathrm{H}), 7.68\left(\mathrm{dd}, J_{l}=7.8 \mathrm{~Hz}, J_{2}=1.6 \mathrm{~Hz}, 1 \mathrm{H}\right), 7.44-7.40(\mathrm{~m}, 1 \mathrm{H}), 7.29$ (dd, $J_{l}=4.3 \mathrm{~Hz}, J_{2}=$ $2.2 \mathrm{~Hz}, 1 \mathrm{H}), 7.04-7.00(\mathrm{~m}, 1 \mathrm{H}), 6.96-6.94(\mathrm{~m}, 2 \mathrm{H}), 6.26$ (d, $J=3.6 \mathrm{~Hz}, 1 \mathrm{H}), 2.55(\mathrm{~d}, J=3.9 \mathrm{~Hz}$, 1H). ${ }^{13} \mathbf{C}$ NMR (100 MHz, $\left.\mathbf{C D C l}_{3}\right) \delta$ 146.37, 145.04, 139.64, 129.88, 128.84, 127.79, 126.89, 125.97, 125.90, 98.05, 75.75. HRMS (ESI) calculated [M-OH] ${ }^{+}$for $\mathrm{C}_{11} \mathrm{H}_{8} \mathrm{IS}: 298.9386$, found: 298.9388. FTIR (cm $\left.{ }^{-1}\right)$ 3744, 3585, 2923, 2363, 1461, 1429, 1005, 853.

\section{Benzofuran-2-yl(2-iodophenyl)methanol (3u)}<smiles>OC(c1cc2ccccc2o1)c1ccccc1I</smiles>

Following the general procedure, treatment of benzofuran-2-carbaldehyde $1 \mathbf{u} \quad(0.073 \quad \mathrm{~g}, \quad 61 \quad \mu \mathrm{L}, \quad 0.5 \quad \mathrm{mmol}), \quad 2$-(trimethylsilyl)phenyl trifluoromethanesulfonate $\mathbf{2 a}(0.373 \mathrm{~g}, 303 \mu \mathrm{L}, 1.25 \mathrm{mmol})$ and $\mathrm{KI}(0.208$ $\mathrm{g}, 1.25 \mathrm{mmol})$ in the presence of $\mathrm{KF}(0.145 \mathrm{~g}, 2.5 \mathrm{mmol})$ and 18 -crown-6 mixture using $4 \mathrm{~N} \mathrm{HCl}(\mathrm{HCl}$ in dioxane, $0.8 \mathrm{~mL})$, and subsequent purification via silica gel flash column chromatography (Pet. ether $/$ EtOAc $=90 / 10$ ) of the crude reaction mixture afforded benzofuran-2-yl(2-iodophenyl)methanol 3u as a brown oil (0.090 g, 51\% yield).

$\boldsymbol{R}_{\mathbf{f}}($ Pet. ether $/$ EtOAc $=85 / 15): 0.45 ;{ }^{1} \mathbf{H}$ NMR (400 MHz, $\left.\mathbf{C D C l}_{3}\right) \delta 7.86\left(\mathrm{dd}, J_{l}=7.9 \mathrm{~Hz}, J_{2}=\right.$ $0.8 \mathrm{~Hz}, 1 \mathrm{H}), 7.66\left(\mathrm{dd}, J_{1}=7.8 \mathrm{~Hz}, J_{2}=1.5 \mathrm{~Hz}, 1 \mathrm{H}\right), 7.52-7.47(\mathrm{~m}, 2 \mathrm{H}), 7.44-7.40(\mathrm{~m}, 1 \mathrm{H}), 7.31-$ $7.26(\mathrm{~m}, 1 \mathrm{H}), 7.24-7.20(\mathrm{~m}, 1 \mathrm{H}), 7.06\left(\mathrm{td}, J_{1}=7.7 \mathrm{~Hz}, J_{2}=1.6 \mathrm{~Hz}, 1 \mathrm{H}\right), 6.46(\mathrm{~s}, 1 \mathrm{H}), 6.17(\mathrm{~s}$, 1H), 2.91 (bs, 1H). ${ }^{13} \mathbf{C}$ NMR (100 MHz, $\left.\mathbf{C D C l}_{3}\right) \delta 157.09,155.23,142.25,139.60,130.14$, $128.76,128.41,128.06,124.58,122.98,121.38,111.51,105.31,98.45,74.18$. HRMS (ESI) calculated $[\mathrm{M}-\mathrm{OH}]^{+}$for $\mathrm{C}_{15} \mathrm{H}_{10} \mathrm{IO}: 332.9771$, found: 332.9777. FTIR $\left(\mathbf{c m}^{-1}\right)$ 3322, 3059, 2364, $1585,1454,1253,1007$.

\section{Cyclohexyl(2-iodophenyl)methanol (3v)}<smiles>OC(c1ccccc1I)C1CCCCC1</smiles>

Following the general procedure, treatment of cyclohexanecarbaldehyde $\mathbf{1 v}$ $(0.056 \quad \mathrm{~g}, \quad 0.061 \mu \mathrm{L}, \quad 0.5 \quad \mathrm{mmol}), \quad$ 2-(trimethylsilyl)phenyl trifluoromethanesulfonate $2 \mathrm{a}(0.373 \mathrm{~g}, 303 \mu \mathrm{L}, 1.25 \mathrm{mmol})$ and $\mathrm{KI}(0.208 \mathrm{~g}$, 
$1.25 \mathrm{mmol})$ in the presence of $\mathrm{KF}(0.145 \mathrm{~g}, 2.5 \mathrm{mmol})$ and 18-crown-6 (0.660 g, $2.5 \mathrm{mmol})$ in THF $(2.0 \mathrm{~mL})$ at $25^{\circ} \mathrm{C}$ for $24 \mathrm{~h}$ followed by quenching the reaction mixture using $4 \mathrm{~N} \mathrm{HCl}(\mathrm{HCl}$ in dioxane, $0.8 \mathrm{~mL}$ ), and subsequent purification via silica gel flash column chromatography (Pet. ether $/$ EtOAc $=90 / 10)$ of the crude reaction mixture afforded cyclohexyl(2iodophenyl)methanol 3v as a white solid (0.066 g, 42\% yield).

$\boldsymbol{R}_{\mathbf{f}}($ Pet. ether $/$ EtOAc $=85 / 15): 0.58 ;{ }^{1} \mathbf{H}$ NMR (400 $\left.\mathbf{M H z}, \mathbf{C D C l}_{3}\right) \delta 7.80\left(\mathrm{dd}, J_{1}=7.9 \mathrm{~Hz}, J_{2}=\right.$ $1.2 \mathrm{~Hz}, 1 \mathrm{H}), 7.44-7.41$ (m, 1H), 7.37-7.33 (m, 1H), 6.98-6.94 (m, 1H),4.71-4.69 (m, 1H), 1.93 $(\mathrm{d}, J=3.5 \mathrm{~Hz}, 1 \mathrm{H}), 1.85-1.65(\mathrm{~m}, 5 \mathrm{H}), 1.48-1.45(\mathrm{~m}, 1 \mathrm{H}), 1.30-1.16(\mathrm{~m}, 5 \mathrm{H}) .{ }^{13} \mathbf{C}$ NMR (100 MHz, $\left.\mathbf{C D C l}_{3}\right) \delta 145.66,139.43,129.18,128.38,128.13,98.95,81.59,44.16,29.86,27.38$, 26.51, 26.16. HRMS (ESI) calculated $[\mathrm{M}-\mathrm{OH}]^{+}$for $\mathrm{C}_{13} \mathrm{H}_{16} \mathrm{I}: 299.0291$, found: 299.0294. FTIR $\left(\mathbf{c m}^{-1}\right)$ 3408, 3056, 2926, 2850, 1613, 1456, 1007.

\section{1-(2-Iodophenyl)-2-methylpropan-1-ol (3w)}<smiles>CC(C)C(O)c1ccccc1I</smiles>

Following the general procedure, treatment of isobutyraldehyde $\mathbf{1 w}(0.036 \mathrm{~g}$, $0.046 \mu \mathrm{L}, 0.5 \mathrm{mmol}$ ), 2-(trimethylsilyl)phenyl trifluoromethanesulfonate $\mathbf{2 a}$ $(0.373 \mathrm{~g}, 303 \mu \mathrm{L}, 1.25 \mathrm{mmol})$ and $\mathrm{KI}(0.208 \mathrm{~g}, 1.25 \mathrm{mmol})$ in the presence of $\mathrm{KF}(0.145 \mathrm{~g}, 2.5 \mathrm{mmol})$ and 18-crown-6 (0.660 g, $2.5 \mathrm{mmol})$ in THF $(2.0 \mathrm{~mL})$ at $25{ }^{\circ} \mathrm{C}$ for $24 \mathrm{~h}$ followed by quenching the reaction mixture using $4 \mathrm{~N} \mathrm{HCl}$ ( $\mathrm{HCl}$ in dioxane, $0.8 \mathrm{~mL}$ ), and subsequent purification via silica gel flash column chromatography (Pet. ether $/$ EtOAc $=90 / 10)$ of the crude reaction mixture afforded 1-(2iodophenyl)-2-methylpropan-1-ol 3w as a colourless oil (0.039 g, 28\% yield).

$\boldsymbol{R}_{\mathbf{f}}($ Pet. ether $/$ EtOAc $=85 / 15): 0.82 ;{ }^{1} \mathbf{H}$ NMR (400 MHz, $\left.\mathbf{C D C l}_{3}\right) \delta 7.81(\mathrm{~d}, J=7.9 \mathrm{~Hz}, 1 \mathrm{H})$, 7.45-7.43 (m, 1H), 7.38-7.34 (m, 1H), 6.98-6.94 (m, 1H), 4.70 (d, $J=5.6$ Hz, 1H), 2.11-1.99 (m, 1H), 1.94 (bs, 1H), 0.98 (d, $J=3.3 \mathrm{~Hz}, 3 \mathrm{H}), 0.98$ (d, $J=3.1 \mathrm{~Hz}, 3 \mathrm{H}) .{ }^{13} \mathbf{C}$ NMR (100 MHz, $\left.\mathbf{C D C l}_{3}\right) \delta 145.89,139.49,129.18,128.39,127.99,98.74,81.97,34.18,19.76,16.65$. HRMS (ESI) calculated $[\mathrm{M}-\mathrm{OH}]^{+}$for $\mathrm{C}_{10} \mathrm{H}_{11} \mathrm{I}$ : 258.9978, found: 258.9979. FTIR (cm $\left.\mathbf{( c m}^{-1}\right)$ 3420, 2962, 1584, 1465, 1209, 1003.

\section{(2-Iodo-4,5-dimethylphenyl)(4-nitrophenyl)methanol (3x)}<smiles>Cc1cc(I)c(C(O)c2ccc([N+](=O)[O-])cc2)cc1C</smiles>

Following the general procedure, treatment of 4nitrobenzaldehyde 1a $(0.038 \mathrm{~g}, 0.25 \mathrm{mmol})$, 4,5-dimethyl-2- 
(trimethylsilyl)phenyl trifluoromethanesulfonate $2 \mathbf{b}(0.204 \mathrm{~g}, 0.625 \mathrm{mmol})$ and $\mathrm{KI}(0.104 \mathrm{~g}$, $0.625 \mathrm{mmol})$ in the presence of KF $(0.073 \mathrm{~g}, 1.25 \mathrm{mmol})$ and 18 -crown-6 (0.330 g, $1.25 \mathrm{mmol})$ in THF $(1.0 \mathrm{~mL})$ at $25^{\circ} \mathrm{C}$ for $24 \mathrm{~h}$ followed by quenching the reaction mixture using $4 \mathrm{~N} \mathrm{HCl}$ $(\mathrm{HCl}$ in dioxane, $0.4 \mathrm{~mL}$ ), and subsequent purification via silica gel flash column chromatography (Pet. ether $/$ EtOAc $=90 / 10)$ of the crude reaction mixture afforded (2-iodo-4,5dimethylphenyl)(4-nitrophenyl)methanol 3x as a yellow oil (0.062 g, 65\% yield).

$\boldsymbol{R}_{\mathbf{f}}($ Pet. ether $/$ EtOAc $=85 / 15): 0.45 ;{ }^{1} \mathbf{H}$ NMR (400 MHz, $\left.\mathbf{C D C l}_{3}\right) \delta$ 8.19-8.16 (m, 2H), 7.6-7.59 (m, 3H), 7.07 (s, 1H), 6.13 (s, 1H), 2.63 (bs, 1H), 2.20 (s, 3H), 2.17 (s, 3H). ${ }^{13}$ C NMR (100 MHz, $\left.\mathbf{C D C l}_{3}\right) \delta 149.93,147.27,142.05,140.32,139.46,138.03,129.56,127.59,123.71,95.02$, 78.01, 19.66, 19.13. HRMS (ESI) calculated [M-OH] ${ }^{+}$for $\mathrm{C}_{15} \mathrm{H}_{13} \mathrm{INO}_{2}$ : 365.9985, found: 365.9992. FTIR (cm $\left.{ }^{-1}\right)$ 3306, 2920, 2363, 1519, 1345, 859.

\section{(6-Iodobenzo[d][1,3]dioxol-5-yl)(4-nitrophenyl)methanol (3y)}<smiles>O=[N+]([O-])c1ccc(C(O)c2cc3c(cc2I)OCO3)cc1</smiles>

3y

Following the general procedure, treatment of 4-nitrobenzaldehyde 1a (0.038 g, $0.25 \mathrm{mmol}), \quad 6$-(trimethylsilyl)benzo[d][1,3]dioxol-5-yl trifluoromethanesulfonate $2 \mathrm{c}(0.214 \mathrm{~g}, 0.625 \mathrm{mmol})$ and $\mathrm{KI}(0.104 \mathrm{~g}$, $0.625 \mathrm{mmol})$ in the presence of $\mathrm{KF}(0.073 \mathrm{~g}, 1.25 \mathrm{mmol})$ and 18 crown-6 (0.330 g, $21.25 \mathrm{mmol})$ in THF $(1.0 \mathrm{~mL})$ at $25{ }^{\circ} \mathrm{C}$ for $24 \mathrm{~h}$ followed by quenching the reaction mixture using $4 \mathrm{~N} \mathrm{HCl}(\mathrm{HCl}$ in dioxane, $0.4 \mathrm{~mL})$, and subsequent purification via silica gel flash column chromatography (Pet. ether $/$ EtOAc $=90 / 10)$ of the crude reaction mixture afforded (6-iodobenzo[d][1,3]dioxol-5-yl)(4-nitrophenyl)methanol 3y as a yellow oil (0.074 g, $74 \%$ yield).

$($ Pet. ether $/$ EtOAc $=85 / 15): 0.35 ;{ }^{1} \mathbf{H}$ NMR (400 MHz, $\left.\mathbf{C D C l}_{3}\right) \delta 8.18(\mathrm{~d}, J=8.6 \mathrm{~Hz}, 2 \mathrm{H}), 7.59$ $(\mathrm{d}, J=8.6 \mathrm{~Hz}, 2 \mathrm{H}), 7.23(\mathrm{~s}, 1 \mathrm{H}), 6.82(\mathrm{~s}, 1 \mathrm{H}), 6.13(\mathrm{~s}, 1 \mathrm{H}), 5.98(\mathrm{~d}, J=1.1 \mathrm{~Hz}, 1 \mathrm{H}), 5.95(\mathrm{~d}, J=$ $1.1 \mathrm{~Hz}, 1 \mathrm{H}), 2.68$ (bs, 1H). ${ }^{13} \mathbf{C}$ NMR (100 MHz, $\left.\mathbf{C D C l}_{3}\right) \delta$ 149.67, 149.23, 148.69, 147.38, 138.37, 127.49, 123.79, 118.67, 108.49, 102.12, 86.65, 78.17. HRMS (ESI) calculated [M-OH] ${ }^{+}$ for $\mathrm{C}_{14} \mathrm{H}_{9} \mathrm{INO}_{4}$ : 381.9571, found: 381.9578. FTIR ( $\left.\mathbf{c m}^{-1}\right)$ 3585, 2900, 2363, 1473, 1345, 1233, $1036,859$. 


\section{(2-Iodo-3,6-dimethylphenyl)(4-nitrophenyl)methanol (3z)}

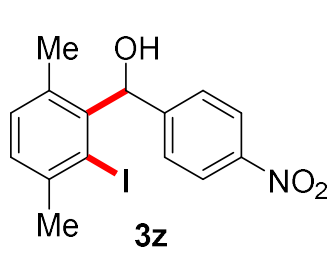

Following the general procedure, treatment of 4-nitrobenzaldehyde 1a (0.038 g, $0.25 \mathrm{mmol}), \quad 3,6$-dimethyl-2-(trimethylsilyl)phenyl trifluoromethanesulfonate $2 \mathbf{d}(0.204 \mathrm{~g}, 0.625 \mathrm{mmol})$ and $\mathrm{KI}(0.104 \mathrm{~g}$, $0.625 \mathrm{mmol})$ in the presence of KF $(0.073 \mathrm{~g}, 1.25 \mathrm{mmol})$ and 18-crown-6 $(0.330 \mathrm{~g}, 1.25 \mathrm{mmol})$ in THF $(1.0 \mathrm{~mL})$ at $25{ }^{\circ} \mathrm{C}$ for $24 \mathrm{~h}$ followed by quenching the reaction mixture using $4 \mathrm{~N} \mathrm{HCl}(\mathrm{HCl}$ in dioxane, $0.4 \mathrm{~mL})$, and subsequent purification via silica gelflash column chromatography (Pet. ether $/$ EtOAc $=85 / 15)$ of the crude reaction mixture afforded $(2-$ iodo-3,6-dimethylphenyl)(4-nitrophenyl)methanol $\mathbf{3 z}$ as a yellow oil (0.052 g, 54\% yield).

$\boldsymbol{R}_{\mathbf{f}}($ Pet. ether $/$ EtOAc $=85 / 15): 0.45 ;{ }^{1} \mathbf{H}$ NMR (400 $\left.\mathbf{M H z}, \mathbf{C D C l}_{3}\right) \delta 8.17-8.14(\mathrm{~m}, 2 \mathrm{H}), 7.50$ $7.43(\mathrm{~m}, 2 \mathrm{H}), 7.14$ (d, $J=7.7 \mathrm{~Hz}, 1 \mathrm{H}), 7.02$ (d, $J=7 \mathrm{~Hz}, 1 \mathrm{H}), 6.76$ (d, $J=4.3 \mathrm{~Hz}, 1 \mathrm{H}), 2.86$ (bs, 1H), 2.51 (s, 3H), 2.11 (s, 3H). ${ }^{13} \mathbf{C}$ NMR (100 MHz, $\left.\mathbf{C D C l}_{3}\right) \delta 149.99,146.90,142.25,140.56$, 136.58, 132.31, 129.93, 126.46, 123.56, 76.84, 30.67, 20.38. HRMS (ESI) calculated [M-OH] ${ }^{+}$ for $\mathrm{C}_{15} \mathrm{H}_{13} \mathrm{INO}_{2}$ : 365.9985, found: 365.9985. FTIR (cm-1) 3583, 2922, 2015, 1598, 1516, 1342, 877.

\section{(3-Iodonaphthalen-2-yl)(4-nitrophenyl)methanol (3aa)}<smiles>O=[N+]([O-])c1ccc(C(O)c2cc3ccccc3cc2I)cc1</smiles>

Following the general procedure, treatment of 4nitrobenzaldehyde $\mathbf{1 a}(0.0378 \mathrm{~g}, \quad 0.25 \quad \mathrm{mmol}), \quad 3-$ (trimethylsilyl)naphthalen-2-yl trifluoromethanesulfonate $\mathbf{2 e}$ $(0.218 \mathrm{~g}, 0.625 \mathrm{mmol})$ and $\mathrm{KI}(0.104 \mathrm{~g}, 0.625 \mathrm{mmol})$ in the presence of KF (0.072 g, $1.25 \mathrm{mmol})$ and 18-crown-6 (0.330

g, $1.25 \mathrm{mmol})$ in THF $(1.0 \mathrm{~mL})$ at $25^{\circ} \mathrm{C}$ for $24 \mathrm{~h}$ followed by quenching the reaction mixture using $4 \mathrm{~N} \mathrm{HCl}(\mathrm{HCl}$ in dioxane, $0.4 \mathrm{~mL})$, and subsequent purification via silica gel flash column chromatography (Pet. ether $/$ EtOAc $=85 / 15$ ) of the crude reaction mixture afforded(3iodonaphthalen-2-yl)(4-nitrophenyl)methanol 3aa as a yellow solid (0.055 g, 55\% yield).

$\boldsymbol{R}_{\mathbf{f}}($ Pet. ether $/$ EtOAc $=85 / 15): 0.46 ;{ }^{1} \mathbf{H}$ NMR (400 MHz, $\left.\mathbf{C D C l}_{3}\right) \delta 8.41(\mathrm{~s}, 1 \mathrm{H}), 8.18(\mathrm{~d}, J=8.8$ $\mathrm{Hz}, 2 \mathrm{H}), 7.85(\mathrm{~s}, 1 \mathrm{H}), 7.78-7.76(\mathrm{~m}, 1 \mathrm{H}), 7.73-7.71(\mathrm{~m}, 1 \mathrm{H}), 7.61(\mathrm{~d}, J=8.5 \mathrm{~Hz}, 2 \mathrm{H}), 7.53-7.49$ (m, 2H), 6.29 (s, 1H), 2.81 (bs, 1H). ${ }^{13} \mathbf{C}$ NMR (100 MHz, $\left.\mathbf{C D C l}_{3}\right) \delta$ 149.30, 147.45, 140.66, 139.52 , 134.66, 132.82, 128.40, 128.26, 127.75, 127.46, 127.29, 126.62, 123.80, 96.30, 78.14. 
HRMS (ESI) calculated $[\mathrm{M}-\mathrm{OH}]^{+}$for $\mathrm{C}_{17} \mathrm{H}_{11} \mathrm{INO}_{2}$ : 387.9834, found: 387.9829. FTIR (cm ${ }^{-}$ 1) $3741,3584,2921,2363,1517,1344,1041,858$.

\section{(2-Iodonaphthalen-1-yl)(4-nitrophenyl)methanol (3ab)}<smiles>O=[N+]([O-])c1ccc(C(O)c2c(I)ccc3ccccc23)cc1</smiles>

$3 a b$

Following the general procedure, treatment of 4-nitrobenzaldehyde 1a $(0.0378 \mathrm{~g}, \quad 0.25 \quad \mathrm{mmol}), \quad 1$-(trimethylsilyl)naphthalen-2-yl trifluoromethanesulfonate $2 \mathbf{f}(0.218 \mathrm{~g}, 0.625 \mathrm{mmol})$ and $\mathrm{KI}(0.104 \mathrm{~g}$, $0.625 \mathrm{mmol})$ in the presence of $\mathrm{KF}(0.072 \mathrm{~g}, 1.25 \mathrm{mmol})$ and 18 crown-6 $(0.330 \mathrm{~g}, 1.25 \mathrm{mmol})$ in THF $(1.0 \mathrm{~mL})$ at $25{ }^{\circ} \mathrm{C}$ for $24 \mathrm{~h}$ followed by quenching the reaction mixture using $4 \mathrm{~N} \mathrm{HCl}(\mathrm{HCl}$ in dioxane, $0.4 \mathrm{~mL}$ ), and subsequent purification via silica gel flash column chromatography (Pet. ether $/$ EtOAc $=85 / 15)$ of the crude reaction mixture afforded (2-iodonaphthalen-1-yl)(4nitrophenyl)methanol 3ab as a yellow solid ( $0.055 \mathrm{~g}, 54 \%$ yield).

$\boldsymbol{R}_{\mathbf{f}}($ Pet. ether $/$ EtOAc $=85 / 15): 0.43 ;{ }^{1} \mathbf{H}$ NMR (400 MHz, $\left.\mathbf{C D C l}_{3}\right) \delta 8.14(\mathrm{~d}, \mathrm{~J}=8.8 \mathrm{~Hz}, 2 \mathrm{H})$, 8.01-7.94 (m, 2H), $7.79(\mathrm{~d}, \mathrm{~J}=8.1 \mathrm{~Hz}, 1 \mathrm{H}), 7.55(\mathrm{t}, \mathrm{J}=9.3 \mathrm{~Hz}, 3 \mathrm{H}), 7.43$ (t, J = 7.6 Hz, 1H), 7.31-7.27 (m, 1H), $6.85(\mathrm{~d}, \mathrm{~J}=3.2 \mathrm{~Hz}, 1 \mathrm{H}), 3.01(\mathrm{~d}, \mathrm{~J}=3.8 \mathrm{~Hz}, 1 \mathrm{H}) .{ }^{13} \mathbf{C}$ NMR (100 MHz, $\left.\mathbf{C D C l}_{3}\right) \delta 150.00,147.05,139.60,136.22,134.69,131.36,131.11,128.88,126.80,126.66$, 126.30, 125.93, 123.79, 100.53, 81.11. HRMS (ESI) calculated $[\mathrm{M}-\mathrm{OH}]^{+}$for $\mathrm{C}_{17} \mathrm{H}_{11} \mathrm{INO}_{2}$ : 387.9829, found: 387.9835. FTIR (cm-1) 3745, 3584, 2363, 1601, 1515, 1342, 1061, 852.

\section{(2-Iodo-6-methoxyphenyl)(4-nitrophenyl)methanol (3ac)}<smiles>COc1cccc(I)c1C(O)c1ccc([N+](=O)[O-])cc1</smiles>

Following the general procedure, treatment of 4-nitrobenzaldehyde 1a (0.038 g, $0.25 \mathrm{mmol}), \quad 3$-methoxy-2-(trimethylsilyl)phenyl trifluoromethanesulfonate $2 \mathrm{~g}(0.205 \mathrm{~g}, 0.625 \mathrm{mmol})$ and $\mathrm{KI}(0.104 \mathrm{~g}$,

$0.625 \mathrm{mmol})$ in the presence of $\mathrm{KF}(0.073 \mathrm{~g}, 1.25 \mathrm{mmol})$ and 18-crown$6(0.330 \mathrm{~g}, 1.25 \mathrm{mmol})$ in THF $(1.0 \mathrm{~mL})$ at $25^{\circ} \mathrm{C}$ for $24 \mathrm{~h}$ followed by quenching the reaction mixture using $4 \mathrm{~N} \mathrm{HCl}(\mathrm{HCl}$ in dioxane, $0.4 \mathrm{~mL})$, and subsequent purification via silica gel flash column chromatography (Pet. ether $/$ EtOAc $=85 / 15)$ of the crude reaction mixture afforded (2iodo-6-methoxyphenyl)(4-nitrophenyl)methanol 3ac as a yellow solid (0.077 g, 79\% yield). $\boldsymbol{R}_{\mathbf{f}}($ Pet. ether $/$ EtOAc $=85 / 15): 0.12 ;{ }^{1} \mathbf{H}$ NMR (400 MHz, $\left.\mathbf{C D C l}_{3}\right) \delta 8.15-8.12(\mathrm{~m}, 2 \mathrm{H}), 7.56$ $\left(\mathrm{dd}, J_{l}=7.9 \mathrm{~Hz}, J_{2}=0.8 \mathrm{~Hz}, 1 \mathrm{H}\right), 7.49(\mathrm{~d}, J=8.2 \mathrm{~Hz}, 2 \mathrm{H}), 7.02(\mathrm{t}, J=8.1 \mathrm{~Hz}, 1 \mathrm{H}), 7.90(\mathrm{~d}, J=8.2$ 
$\mathrm{Hz}, 1 \mathrm{H}), 6.23(\mathrm{~d}, J=11.3 \mathrm{~Hz}, 1 \mathrm{H}), 4.12$ (d, $J=11.4 \mathrm{~Hz}, 1 \mathrm{H}), 3.68(\mathrm{~s}, 3 \mathrm{H}) .{ }^{\mathbf{1 3}} \mathbf{C}$ NMR (100 MHz, $\left.\mathbf{C D C l}_{3}\right) \delta 157.28,151.18,147.05,132.95,132.79,130.91,126.28,123.44,112.31,100.78$, 79.69, 55.91. HRMS (ESI) calculated $[\mathrm{M}-\mathrm{OH}]^{+}$for $\mathrm{C}_{14} \mathrm{H}_{11} \mathrm{INO}_{3}$ : 367.9778, found: 367.9786 . FTIR (cm $\left.{ }^{-1}\right)$ 3434, 2722, 1567, 1517, 1344, 1024.

(2-Iodo-4-methylphenyl)(4-nitrophenyl)methanol (3ad) and (2-Iodo-5-methylphenyl)(4nitrophenyl)methanol (3ad')<smiles>Cc1ccc(C(O)c2ccc([N+](=O)[O-])cc2)c(I)c1</smiles>
Following the general procedure, treatment of 4-nitrobenzaldehyde 1a $(0.038 \mathrm{~g}, 0.25 \mathrm{mmol})$, 4-methyl-2-(trimethylsilyl)phenyl trifluoromethanesulfonate $\mathbf{2 h}(0.195$ g,0.625 $\mathrm{mmol})$ and $\mathrm{KI}(0.104 \mathrm{~g}, 0.625 \mathrm{mmol})$ in the presence of $\mathrm{KF}(0.073 \mathrm{~g}, 1.25 \mathrm{mmol})$ and 18-crown-6 $(0.330 \mathrm{~g}, 1.25 \mathrm{mmol})$ in THF $(1.0 \mathrm{~mL})$ at $25{ }^{\circ} \mathrm{C}$ for $24 \mathrm{~h}$ followed by quenching the reaction mixture using $4 \mathrm{~N} \mathrm{HCl}(\mathrm{HCl}$ in dioxane, $0.4 \mathrm{~mL})$, and subsequent purification via silica gelflash column chromatography (Pet. ether $/$ EtOAc $=90 / 10$ ) of the crude reaction mixture afforded inseparable (1:1) regioisomeric mixture of (2-iodo-4-methylphenyl)(4-nitrophenyl)methanol (3ad) and (2-iodo-5-methylphenyl)(4-nitrophenyl)methanol (3ad') as a yellow oil (0.046 g, 49\% yield).

$\boldsymbol{R}_{\mathbf{f}}($ Pet. ether $/$ EtOAc $=85 / 15): 0.35 ;{ }^{1} \mathbf{H}$ NMR (400 $\left.\mathbf{M H z}, \mathbf{C D C l}_{3}\right) \delta$ 7.73-7.10 (m, 1H), 7.62$7.58(\mathrm{~m}, 4 \mathrm{H}), 7.17-7.15(\mathrm{~m}, 1 \mathrm{H}), 6.85\left(\mathrm{dd}, J_{1}=8.1 \mathrm{~Hz}, J_{2}=1.8 \mathrm{~Hz}, 1 \mathrm{H}\right), 6.16(\mathrm{~s}, 1 \mathrm{H}), 2.64-$ 2.61 (m, 1H), 2.28 (s, 3H). ${ }^{13} \mathbf{C}$ NMR (100 MHz, $\left.\mathbf{C D C l}_{3}\right) \delta 20.64,78.08,94.68,123.75,127.73$, $128.71,129.99,139.35,140.28,141.80,147.37,149.60$. Representative peak for other isomer:

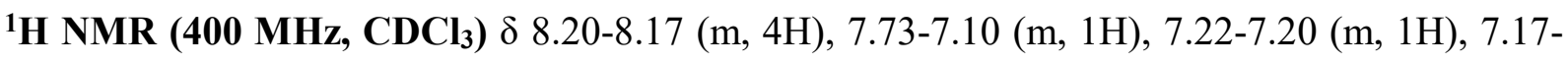
$7.15(\mathrm{~m}, 1 \mathrm{H}), 6.16(\mathrm{~s}, 1 \mathrm{H}), 2.64-2.61(\mathrm{~m}, 1 \mathrm{H}), 2.30(\mathrm{~s}, 3 \mathrm{H}) .{ }^{13} \mathbf{C}$ NMR (100 MHz, CDCl $) \delta$ 21.18, 78.18, 98.72, 123.78, 127.75, 129.40, 131.34, 139.62, 140.53, 144.39, 147.40, 149.75. HRMS (ESI) calculated $[\mathrm{M}-\mathrm{OH}]{ }^{+}$for $\mathrm{C}_{14} \mathrm{H}_{11} \mathrm{INO}_{2}: 351.9829$, found: 351.9834 . FTIR (cm 1) $3399,2853,1600,1518,1345,1011$.

\section{(2-Bromophenyl)(4-nitrophenyl)methanol (3ae)}<smiles>O=[N+]([O-])c1ccc(C(O)c2ccccc2Br)cc1</smiles>

Following the general procedure, treatment of 4-nitrobenzaldehyde 1a (0.038 g, $0.25 \mathrm{mmol}), 2$-(trimethylsilyl)phenyl trifluoromethanesulfonate 
2a $(0.186 \mathrm{~g}, 152 \mu \mathrm{L}, 0.625 \mathrm{mmol})$ and $\mathrm{KBr}(0.074 \mathrm{~g}, 0.625 \mathrm{mmol})$ in the presence of $\mathrm{KF}(0.073$ $\mathrm{g}, 1.25 \mathrm{mmol})$ and 18 -crown-6 $(0.330 \mathrm{~g}, 1.25 \mathrm{mmol})$ in THF $(1.0 \mathrm{~mL})$ at $25^{\circ} \mathrm{C}$ for $24 \mathrm{~h}$ followed by quenching the reaction mixture using $4 \mathrm{~N} \mathrm{HCl}(\mathrm{HCl}$ in dioxane, $0.4 \mathrm{~mL})$, and subsequent purification via silica gel flash column chromatography (Pet. ether $/$ EtOAc $=90 / 10)$ of the crude reaction mixture afforded (2-bromophenyl)(4-nitrophenyl)methanol 3ae as a yellow solid (0.057 g, 74\% yield).

$\boldsymbol{R}_{\mathbf{f}}($ Pet. ether $/$ EtOAc $=85 / 15): 0.39 ;{ }^{1} \mathbf{H}$ NMR (400 MHz, $\left.\mathbf{C D C l}_{3}\right) \delta 8.17(\mathrm{~d}, J=8.8 \mathrm{~Hz}, 2 \mathrm{H})$, 7.60-7.55 (m, 3H), $7.46\left(\mathrm{dd}, J_{1}=7.8 \mathrm{~Hz}, J_{2}=1.7 \mathrm{~Hz}, 1 \mathrm{H}\right), 7.34\left(\mathrm{td}, J_{1}=7.6 \mathrm{~Hz}, J_{2}=1.1 \mathrm{~Hz}\right.$, 1H), $7.19\left(\mathrm{td}, J_{1}=7.6 \mathrm{~Hz}, J_{2}=1.7 \mathrm{~Hz}, 1 \mathrm{H}\right), 6.30$ (s, 1H), 2.77 (bs, 1H). ${ }^{13} \mathbf{C}$ NMR (100 MHz, $\left.\mathbf{C D C l}_{3}\right) \delta 149.41,147.36,141.65,133.20,129.95,128.72,128.26,127.72,123.78,122.79$, 73.93. HRMS (ESI) calculated $[\mathrm{M}-\mathrm{OH}]^{+}$for $\mathrm{C}_{13} \mathrm{H}_{9} \mathrm{BrNO}_{2}: 289.9811$, found: 289.9814. Isotopic Mass: [M-OH+2] 291.9799. FTIR (cm-1) 3584, 3072, 2364, 1518, 1345, 1016, 864.

\section{(2-Chlorophenyl)(4-nitrophenyl)methanol (3af)}<smiles>O=[N+]([O-])c1ccc(C(O)c2ccccc2Cl)cc1</smiles>

Following the general procedure, treatment of 4-nitrobenzaldehyde 1a (0.038 g, $0.25 \mathrm{mmol}), 2$-(trimethylsilyl)phenyl trifluoromethanesulfonate 2a $(0.186 \mathrm{~g}, 152 \mu \mathrm{L}, 0.625 \mathrm{mmol})$ and $\mathrm{KCl}(0.047 \mathrm{~g}, 0.625 \mathrm{mmol})$ in the presence of KF (0.073 g, $1.25 \mathrm{mmol})$ and 18-crown-6 (0.330 g, 1.25 $\mathrm{mmol})$ in THF $(1.0 \mathrm{~mL})$ at $25^{\circ} \mathrm{C}$ for $24 \mathrm{~h}$ followed by quenching the reaction mixture using $4 \mathrm{~N}$ $\mathrm{HCl}$ ( $\mathrm{HCl}$ in dioxane, $0.4 \mathrm{~mL}$ ), and subsequent purification via silica gel flash column chromatography (Pet. ether $/$ EtOAc $=90 / 10$ ) of the crude reaction mixture afforded (2chlorophenyl)(4-nitrophenyl)methanol 3af as a yellow solid (0.042 g, 62\% yield).

$\boldsymbol{R}_{\mathbf{f}}($ Pet. ether $/$ EtOAc $=85 / 15): 0.39{ }^{1} \mathbf{H}$ NMR (400 MHz, $\left.\mathbf{C D C l}_{3}\right) \delta 8.18(\mathrm{~d}, J=8.8 \mathrm{~Hz}, 2 \mathrm{H})$, $7.59(\mathrm{~d}, J=8.8 \mathrm{~Hz}, 2 \mathrm{H}), 7.49\left(\mathrm{dd}, J_{1}=7.6 \mathrm{~Hz}, J_{2}=1.6 \mathrm{~Hz}, 1 \mathrm{H}\right), 7.38\left(\mathrm{dd}, J_{1}=7.7 \mathrm{~Hz}, J_{2}=1.4\right.$ $\mathrm{Hz}, 1 \mathrm{H}), 7.33-7.24(\mathrm{~m}, 2 \mathrm{H}), 6.34(\mathrm{~d}, J=2.7 \mathrm{~Hz}, 1 \mathrm{H}), 2.63$ (d, $J=3.6 \mathrm{~Hz}, 1 \mathrm{H}) .{ }^{13} \mathbf{C}$ NMR (100 MHz, $\left.\mathbf{C D C l}_{3}\right) \delta 149.40,147.28,140.00,132.43,129.83,129.52,128.19,127.55,127.53,123.69$, 71.71. HRMS (ESI) calculated $[\mathrm{M}-\mathrm{OH}]^{+}$for $\mathrm{C}_{13} \mathrm{H}_{9} \mathrm{ClNO}_{2}: 246.0316$, found: 246.0322. Isotopic Mass: [M-OH+2] 248.0296. FTIR (cm-1) 3584, 3072, 2363, 1519, 1345, 1025, 865. 


\section{(2-Bromophenyl)(phenyl)methanol (3ag) ${ }^{5}$}

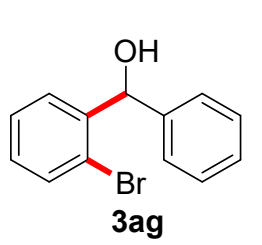

Following the general procedure, treatment of benzaldehyde $\mathbf{1 b}(0.053 \mathrm{~g}, 0.051$ $\mu \mathrm{L}, 0.5 \mathrm{mmol}), 2$-(trimethylsilyl)phenyl trifluoromethanesulfonate $\mathbf{2 a}(0.373 \mathrm{~g}$, $303 \mu \mathrm{L}, 1.25 \mathrm{mmol})$ and $\mathrm{KBr}(0.149 \mathrm{~g}, 1.25 \mathrm{mmol})$ in the presence of $\mathrm{KF}(0.145$ $\mathrm{g}, 2.5 \mathrm{mmol})$ and 18 -crown-6 $(0.660 \mathrm{~g}, 2.5 \mathrm{mmol})$ in THF $(2.0 \mathrm{~mL})$ at $25^{\circ} \mathrm{C}$ for $24 \mathrm{~h}$ followed by quenching the reaction mixture using $4 \mathrm{~N} \mathrm{HCl}(\mathrm{HCl}$ in dioxane, $0.8 \mathrm{~mL})$, and subsequent purification via silica gel flash column chromatography (Pet. ether $/$ EtOAc $=90 / 10$ ) of the crude reaction mixture afforded (2-bromophenyl)(phenyl)methanol 3ag as a yellow oil (0.100 g, 76\% yield).

$\boldsymbol{R}_{\mathbf{f}}($ Pet. ether $/$ EtOAc $=85 / 15)$ : 0.56; ${ }^{1} \mathbf{H}$ NMR (400 $\left.\mathbf{M H z}, \mathbf{C D C l}_{3}\right) \delta$ 7.60-7.54 (m, 2H), 7.42$7.40(\mathrm{~m}, 2 \mathrm{H}), 7.37-7.27(\mathrm{~m}, 4 \mathrm{H}), 7.16\left(\mathrm{td}, J_{l}=7.7 \mathrm{~Hz}, J_{2}=1.7 \mathrm{~Hz}, 1 \mathrm{H}\right), 6.19(\mathrm{~d}, J=2.8 \mathrm{~Hz}, 1 \mathrm{H})$, $2.57(\mathrm{~d}, J=2.8 \mathrm{~Hz}, 1 \mathrm{H}) .{ }^{13} \mathbf{C}$ NMR (100 MHz, $\left.\mathbf{C D C l}_{3}\right) \delta 142.59,142.24,132.94,129.21$, 128.58, 128.57, 127.88, 127.83, 127.16, 122.89, 74.85. HRMS (ESI) calculated $[\mathrm{M}-\mathrm{OH}]^{+}$for $\mathrm{C}_{13} \mathrm{H}_{10} \mathrm{Br}: 244.9960$, found: 244.9967. Isotopic Mass: [M-OH+2] ${ }^{+}$246.9947. FTIR (cm $\left.{ }^{-1}\right)$ 3355, $3061,2363,1437,1181,1013$.

\section{(2-Chlorophenyl)(phenyl)methanol (3ah) ${ }^{5}$}<smiles>OC(c1ccccc1)c1ccccc1Cl</smiles>

Following the general procedure, treatment of benzaldehyde $\mathbf{1 b}(0.027 \mathrm{~g}$, $0.026 \mu \mathrm{L}, 0.25 \mathrm{mmol}), 2$-(trimethylsilyl)phenyl trifluoromethanesulfonate 2a $(0.186 \mathrm{~g}, 152.0 \mu \mathrm{L}, 0.625 \mathrm{mmol})$ and $\mathrm{KCl}(0.047 \mathrm{~g}, 0.625 \mathrm{mmol})$ in the presence of KF (0.073 g, $1.25 \mathrm{mmol})$ and 18-crown-6 (0.330 g, $1.25 \mathrm{mmol})$ in THF $(1.0 \mathrm{~mL})$ at $25^{\circ} \mathrm{C}$ for $24 \mathrm{~h}$ followed by quenching the reaction mixture using $4 \mathrm{~N} \mathrm{HCl}(\mathrm{HCl}$ in dioxane, $0.4 \mathrm{~mL}$ ), and subsequent purification via silica gel flash column chromatography (Pet. ether $/$ EtOAc $=90 / 10)$ of the crude reaction mixture afforded (2chlorophenyl)(phenyl)methanol 3ah as a white solid ( $0.030 \mathrm{~g}, 54 \%$ yield).

$\boldsymbol{R}_{\mathbf{f}}($ Pet. ether $/$ EtOAc $=85 / 15)$ : 0.45; ${ }^{1} \mathbf{H}$ NMR (400 MHz, $\left.\mathbf{C D C l}_{3}\right) \delta$ 7.62-7.60 (m, 2H), 7.41$7.39(\mathrm{~m}, 2 \mathrm{H}), 7.36-7.27(\mathrm{~m}, 4 \mathrm{H}), 7.26-7.21(\mathrm{~m}, 1 \mathrm{H}), 6.23(\mathrm{~d}, J=3.7 \mathrm{~Hz}, 1 \mathrm{H}), 2.40$ (d, $J=3.8 \mathrm{~Hz}$, 1H). ${ }^{13}$ C NMR (100 MHz, $\left.\mathbf{C D C l}_{3}\right) \delta$ 142.35, 141.11, 132.65, 129.69, 128.91, 128.63, 128.17, 127.93, 127.24, 127.06, 72.83. HRMS (ESI) calculated $[\mathrm{M}-\mathrm{OH}]^{+}$for $\mathrm{C}_{13} \mathrm{H}_{10} \mathrm{Cl}: 201.0466$,

\footnotetext{
${ }^{5}$ Kuriyama, M.; Shimazawa, R.; Enomoto, T.; Shirai, R. J. Org. Chem.2008, 73, 6939.
} 
found: 201.0473. Isotopic Mass: $[\mathrm{M}-\mathrm{OH}+2]^{+}$203.0444. FTIR $\left(\mathbf{c m}^{-1}\right)$ 3324, 3029, 2364, 1598, $1443,1019$.

\section{Benzofuran-2-yl(2-bromophenyl)methanol (3ai)}<smiles>OC(c1cc2ccccc2o1)c1ccccc1Br</smiles>

Following the general procedure, treatment of benzofuran-2-carbaldehyde $1 \mathrm{u} \quad(0.037 \quad \mathrm{~g}, \quad 30 \quad \mu \mathrm{L}, \quad 0.25 \quad \mathrm{mmol}), \quad$ 2-(trimethylsilyl)phenyl trifluoromethanesulfonate $2 \mathrm{a}(0.186 \mathrm{~g}, 152.0 \mu \mathrm{L}, 0.625 \mathrm{mmol})$ and $\mathrm{KBr}$ $(0.074 \mathrm{~g}, 0.625 \mathrm{mmol})$ in the presence of $\mathrm{KF}(0.073 \mathrm{~g}, 1.25 \mathrm{mmol})$ and 18 crown-6 $(0.330 \mathrm{~g}, 1.25 \mathrm{mmol})$ in THF $(1.0 \mathrm{~mL})$ at $25{ }^{\circ} \mathrm{C}$ for $24 \mathrm{~h}$ followed by quenching the reaction mixture using $4 \mathrm{~N} \mathrm{HCl}(\mathrm{HCl}$ in dioxane, $0.4 \mathrm{~mL})$, and subsequent purification via silica gel flash column chromatography (Pet. ether $/$ EtOAc $=90 / 10$ ) of the crude reaction mixture afforded benzofuran-2-yl(2-bromophenyl)methanol 3ai as a yellow oil (0.055 g, 71\% yield). $\boldsymbol{R}_{\mathbf{f}}($ Pet. ether $/$ EtOAc $=85 / 15): 0.48 ;{ }^{1} \mathbf{H}$ NMR (400 $\left.\mathbf{M H z}, \mathbf{C D C l}_{3}\right) \delta 7.70\left(\mathrm{dd}, J_{l}=7.8 \mathrm{~Hz}, J_{2}=\right.$ $1.6 \mathrm{~Hz}, 1 \mathrm{H}), 7.58\left(\mathrm{dd}, J_{1}=8.0 \mathrm{~Hz}, J_{2}=1.2 \mathrm{~Hz}, 1 \mathrm{H}\right), 7.52-7.46(\mathrm{~m}, 2 \mathrm{H}), 7.41-7.37(\mathrm{~m}, 1 \mathrm{H}), 7.30-$ $7.26(\mathrm{~m}, 1 \mathrm{H}), 7.25-7.19(\mathrm{~m}, 2 \mathrm{H}), 6.47(\mathrm{~s}, 1 \mathrm{H}), 6.33$ (d, $J=4.3 \mathrm{~Hz}, 1 \mathrm{H}), 2.82$ (d, $J=4.4 \mathrm{~Hz}, 1 \mathrm{H})$. ${ }^{13}$ C NMR (100 MHz, CDCl $) \delta$ 157.05, 155.22, 139.37, 132.96, 129.91, 128.73, 128.08, 127.95, 124.58, 122.99, 122.91, 121.37, 111.51, 104.97, 69.67. HRMS (ESI) calculated [M-OH] ${ }^{+}$for $\mathrm{C}_{15} \mathrm{H}_{10} \mathrm{BrO}: 284.9910$, found: 284.9918 . Isotopic Mass: $[\mathrm{M}-\mathrm{OH}+2]^{+}$286.9898. FTIR (cm 1) $3323,3062,2363,1568,1451,1253,1016$.

\section{Benzofuran-2-yl(2-chlorophenyl)methanol (3aj) ${ }^{6}$}<smiles>OC(c1cc2ccccc2o1)c1ccccc1Cl</smiles>

3aj

Following the general procedure, treatment of benzofuran-2-carbaldehyde $1 \mathrm{u} \quad(0.037 \quad \mathrm{~g}, \quad 30 \quad \mu \mathrm{L}, \quad 0.25 \quad \mathrm{mmol}), \quad$ 2-(trimethylsilyl)phenyl trifluoromethanesulfonate $2 \mathrm{a}(0.186 \mathrm{~g}, 152.0 \mu \mathrm{L}, 0.625 \mathrm{mmol})$ and $\mathrm{KCl}$ $(0.047 \mathrm{~g}, 0.625 \mathrm{mmol})$ in the presence of KF $(0.073 \mathrm{~g}, 1.25 \mathrm{mmol})$ and 18 crown-6 $(0.330 \mathrm{~g}, 1.25 \mathrm{mmol})$ in THF $(1.0 \mathrm{~mL})$ at $25{ }^{\circ} \mathrm{C}$ for $24 \mathrm{~h}$ followed by quenching the reaction mixture using $4 \mathrm{~N} \mathrm{HCl}(\mathrm{HCl}$ in dioxane, $0.4 \mathrm{~mL})$, and subsequent purification via silica gel flash column chromatography (Pet. ether $/$ EtOAc $=90 / 10$ ) of the crude reaction mixture afforded benzofuran-2-yl(2-chlorophenyl)methanol 3aj as a yellow oil (0.031 g, 48\% yield).

\footnotetext{
${ }^{6}$ Xuan, Q.; Kong, W.; Song, Q. J. Org. Chem. 2017, 82, 7602.
} 
$\boldsymbol{R}_{\mathbf{f}}\left(\right.$ Pet. ether $/$ EtOAc $=$ 95/15): 0.40; ${ }^{1} \mathbf{H}$ NMR (400 $\left.\mathbf{M H z}, \mathbf{C D C l}_{3}\right) \delta$ 7.71-7.69 (m, 1H), 7.52$7.50(\mathrm{~m}, 1 \mathrm{H}), 7.48-7.45(\mathrm{~m}, 1 \mathrm{H}), 7.41-7.37$ (m, 1H), 7.36-7.26 (m, 3H), 7.23-7.19 (m, 1H), 6.48 (s, 1H), 6.37 (d, $J=4.4 \mathrm{~Hz}, 1 \mathrm{H}), 2.74(\mathrm{~d}, J=4.4 \mathrm{~Hz}, 1 \mathrm{H}) .{ }^{13} \mathbf{C}$ NMR (100 MHz, CDCl $) \delta$ $157.08,155.22$, 137.80, 132.85, 129.71, 129.62, 128.46, 128.09, 127.35, 124.58, 123.01, 121.37, 111.51, 104.76, 67.41. HRMS (ESI) calculated [M-OH] ${ }^{+}$for $\mathrm{C}_{15} \mathrm{H}_{10} \mathrm{ClO}$ : 241.0415, found: 241.0420. Isotopic Mass: [M-OH+2] 243.0392. FTIR (cm $\left.{ }^{-1}\right)$ 3322, 3064, 2363, 1581, 1450, $1251,1024$.

\section{1-(2-Bromophenyl)-2-methylpropan-1-ol (3ak) ${ }^{7}$}

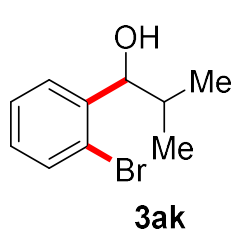

Following the general procedure, treatment of isobutyraldehyde $1 \mathbf{w}(0.036 \mathrm{~g}$, $0.046 \mu \mathrm{L}, 0.5 \mathrm{mmol}$ ), 2-(trimethylsilyl)phenyl trifluoromethanesulfonate $2 \mathrm{a}$ $(0.373 \mathrm{~g}, 303 \mu \mathrm{L}, 1.25 \mathrm{mmol})$ and $\mathrm{KBr}(0.149 \mathrm{~g}, 1.25 \mathrm{mmol})$ in the presence of $\mathrm{KF}(0.145 \mathrm{~g}, 2.5 \mathrm{mmol})$ and 18-crown-6 (0.660 g, $2.5 \mathrm{mmol})$ in THF $(2.0 \mathrm{~mL})$ at $25{ }^{\circ} \mathrm{C}$ for $24 \mathrm{~h}$ followed by quenching the reaction mixture using $4 \mathrm{~N} \mathrm{HCl}(\mathrm{HCl}$ in dioxane, 0.8 $\mathrm{mL}$ ), and subsequent purification via silica gel flash column chromatography (Pet. ether /EtOAc $=90 / 10)$ of the crude reaction mixture afforded 1-(2-bromophenyl)-2-methylpropan-1-ol 3ak as a yellow oil ( $0.063 \mathrm{~g}, 55 \%$ yield).

$\boldsymbol{R}_{\mathbf{f}}\left(\right.$ Pet. ether $/$ EtOAc $=$ 85/15): 0.81; ${ }^{1} \mathbf{H}$ NMR (400 $\left.\mathbf{M H z}, \mathbf{C D C l}_{3}\right) \delta$ 7.53-7.48 (m, 2H), 7.34$7.31(\mathrm{~m}, 1 \mathrm{H}), 7.12\left(\mathrm{td}, J_{l}=7.7 \mathrm{~Hz}, J_{2}=1.6 \mathrm{~Hz}, 1 \mathrm{H}\right), 4.87\left(\mathrm{~d}, J_{l}=5.4 \mathrm{~Hz}, J_{2}=3.2 \mathrm{~Hz}, 1 \mathrm{H}\right), 2.13-$ $2.01(\mathrm{~m}, 1 \mathrm{H}), 1.90(\mathrm{~d}, J=3.5 \mathrm{~Hz}, 1 \mathrm{H}), 0.97(\mathrm{~d}, J=6.9 \mathrm{~Hz}, 1 \mathrm{H}), 0.95(\mathrm{~d}, J=6.9 \mathrm{~Hz}, 1 \mathrm{H}) .{ }^{13} \mathbf{C}$ NMR (100 MHz, CDCl $)$ ) $143.03,132.79,128.79,128.39,127.55,122.80,77.71,34.11,19.61$, 16.92. FTIR (cm $\left.{ }^{-1}\right)$ 3420, 2963, 2871, 1466, 1199, 1007.

\section{(6-Bromobenzo[d][1,3]dioxol-5-yl)(4-nitrophenyl)methanol (3al)}

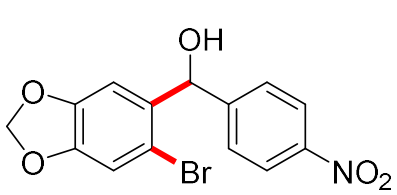

3al

Following the general procedure, treatment of 4-nitrobenzaldehyde 1a (0.038 g, $0.25 \mathrm{mmol}), \quad 6$-(trimethylsilyl)benzo[d][1,3]dioxol-5-yl trifluoromethanesulfonate $2 \mathrm{c}(0.214 \mathrm{~g}, 0.625 \mathrm{mmol})$ and $\mathrm{KBr}(0.074 \mathrm{~g}$, $0.625 \mathrm{mmol})$ in the presence of $\mathrm{KF}(0.073 \mathrm{~g}, 1.25 \mathrm{mmol})$ and 18 crown-6 $(0.330 \mathrm{~g}, 21.25 \mathrm{mmol})$ in THF $(1.0 \mathrm{~mL})$ at $25^{\circ} \mathrm{C}$ for $24 \mathrm{~h}$ followed by quenching the reaction mixture using $4 \mathrm{~N} \mathrm{HCl}(\mathrm{HCl}$ in dioxane, $0.4 \mathrm{~mL})$, and subsequent purification via silica

\footnotetext{
${ }^{7}$ Faggyas, R. J.; E. Calder, D. D.; Wilson, C.; Sutherland, A. J. Org. Chem. 2017, 82, 11585.
} 
gel flash column chromatography (Pet. ether $/$ EtOAc $=90 / 10$ ) of the crude reaction mixture afforded (6-bromobenzo[d][1,3]dioxol-5-yl)(4-nitrophenyl)methanol 3al as a yellow oil (0.070 g, $79 \%$ yield).

$\boldsymbol{R}_{\mathbf{f}}$ (Pet. ether $/$ EtOAc $\left.=85 / 15\right)$ : $0.34{ }^{1} \mathbf{H}$ NMR (400 MHz, $\left.\mathbf{C D C l}_{3}\right) \delta 8.19-8.16(\mathrm{~m}, 2 \mathrm{H}), 7.58(\mathrm{~d}$, $J=8.9 \mathrm{~Hz}, 2 \mathrm{H}), 6.98(\mathrm{~s}, 1 \mathrm{H}), 6.87(\mathrm{~s}, 1 \mathrm{H}), 6.25(\mathrm{~s}, 1 \mathrm{H}), 5.98(\mathrm{~d}, J=1.3 \mathrm{~Hz}, 1 \mathrm{H}), 5.95(\mathrm{~d}, J=1.3$ Hz, 1H), 2.72 (bs, 1H). ${ }^{13} \mathbf{C}$ NMR (100 MHz, CDCl $) \delta$ 149.63, 148.44, 148.16, 147.34, 135.12, 127.39, 123.79, 113.27, 112.76, 108.28, 102.18, 73.67. HRMS (ESI) calculated $[\mathrm{M}-\mathrm{OH}]^{+}$for $\mathrm{C}_{14} \mathrm{H}_{9} \mathrm{BrNO}_{4}$ : 333.9709 , found: 333.9713 . Isotopic Mass: [M-OH+2] 335.9693 . FTIR (cm 1) $3584,2916,2363,1474,1346,1235,1036,861$.

\section{(6-Chlorobenzo[d][1,3]dioxol-5-yl)(4-nitrophenyl)methanol (3am)}<smiles>O=[N+]([O-])c1ccc(C(O)c2cc3c(cc2Cl)OCO3)cc1</smiles>

3am

Following the general procedure, treatment of 4-nitrobenzaldehyde 1a $(0.038 \mathrm{~g}, 0.25 \mathrm{mmol})$, 6-(trimethylsilyl)benzo[d][1,3]dioxol-5-yl trifluoromethanesulfonate $2 \mathrm{c}(0.214 \mathrm{~g}, 0.625 \mathrm{mmol})$ and $\mathrm{KCl}(0.047$ $\mathrm{g}, 0.625 \mathrm{mmol})$ in the presence of KF $(0.073 \mathrm{~g}, 1.25 \mathrm{mmol})$ and 18 crown-6 $(0.330 \mathrm{~g}, 21.25 \mathrm{mmol})$ in THF $(1.0 \mathrm{~mL})$ at $25{ }^{\circ} \mathrm{C}$ for $24 \mathrm{~h}$ followed by quenching the reaction mixture using $4 \mathrm{~N} \mathrm{HCl}(\mathrm{HCl}$ in dioxane, $0.4 \mathrm{~mL})$, and subsequent purification via silica gel flash column chromatography (Pet. ether $/$ EtOAc $=90 / 10$ ) of the crude reaction mixture afforded (6-chlorobenzo[d][1,3]dioxol-5-yl)(4-nitrophenyl)methanol 3am as a yellow solid (0.043g, 56\% yield).

$\boldsymbol{R}_{\mathbf{f}}($ Pet. ether $/$ EtOAc $=85 / 15): 0.34{ }^{1} \mathbf{H}$ NMR (400 MHz, $\left.\mathbf{C D C l}_{3}\right) \delta 8.20-8.17(\mathrm{~m}, 2 \mathrm{H}), 7.58(\mathrm{~d}$, $J=8.9 \mathrm{~Hz}, 2 \mathrm{H}), 6.89$ (s, 1H), $6.83(\mathrm{~s}, 1 \mathrm{H}), 6.28(\mathrm{~s}, 1 \mathrm{H}), 5.99$ (d, $J=1.3 \mathrm{~Hz}, 1 \mathrm{H}), 5.96$ (d, $J=1.3$ $\mathrm{Hz}, 1 \mathrm{H}), 2.55$ (bs, 1H). ${ }^{13} \mathbf{C}$ NMR (100 MHz, $\left.\mathbf{C D C l}_{3}\right) \delta 149.68,148.19,147.52,147.37,133.52$, 127.33, 124.40, 123.82, 109.94, 107.72, 102.18, 71.46. HRMS (ESI) calculated $[\mathrm{M}-\mathrm{OH}]^{+}$for $\mathrm{C}_{14} \mathrm{H}_{9} \mathrm{ClNO}_{4}:$ : 290.0215, found: 290.0219. Isotopic Mass: [M-OH+2] ${ }^{+}$292.0193. FTIR (cm ${ }^{-}$ 1) $3584,2918,2364,1475,1346,1240,1036,863$.

\section{3-Hydroxy-3-(2-iodophenyl)-1-methylindolin-2-one (5a)}

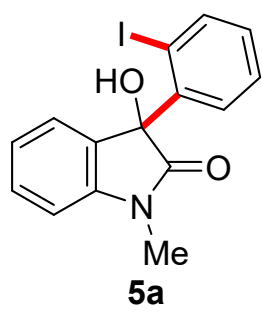

Following the general procedure, treatment of 1-methylindoline-2,3-dione 4a $(0.040 \mathrm{~g}, 0.25 \mathrm{mmol}), 2$-(trimethylsilyl)phenyl trifluoromethanesulfonate 2a $(0.187 \mathrm{~g}, 152 \mu \mathrm{L}, 0.625 \mathrm{mmol})$ and $\mathrm{KI}(0.104 \mathrm{~g}, 0.625 \mathrm{mmol})$ in the presence 
of KF (0.072 g, $1.25 \mathrm{mmol})$ and 18-crown-6 (0.330 g, $1.25 \mathrm{mmol})$ in THF $(1.0 \mathrm{~mL})$ at $60{ }^{\circ} \mathrm{C}$ for $24 \mathrm{~h}$ followed by quenching the reaction mixture using $4 \mathrm{~N} \mathrm{HCl}(\mathrm{HCl}$ in dioxane, $0.4 \mathrm{~mL})$, and subsequent purification via silica gel flash column chromatography (Pet. ether $/$ EtOAc $=90 / 10$ ) of the crude reaction mixture afforded 3-hydroxy-3-(2-iodophenyl)-1-methylindolin-2-one 5a as a yellow solid ( $0.045 \mathrm{~g}, 49 \%$ yield).

$\boldsymbol{R}_{\mathbf{f}}($ Pet. ether $/$ EtOAc $=85 / 15): 0.23 ;{ }^{1} \mathbf{H}$ NMR (400 $\left.\mathbf{M H z}, \mathbf{C D C l}_{3}\right) \delta 8.12(\mathrm{~d}, J=7.9 \mathrm{~Hz}, 1 \mathrm{H})$, $7.80(\mathrm{~d}, \mathrm{~J}=7.5 \mathrm{~Hz}, 1 \mathrm{H}), 7.50-7.46(\mathrm{~m}, 1 \mathrm{H}), 7.40-7.36(\mathrm{~m}, 1 \mathrm{H}), 7.05-6.95(\mathrm{~m}, 3 \mathrm{H}), 6.90$ (d, $J=$ $7.42 \mathrm{~Hz}, 1 \mathrm{H}), 3.39$ (bs, 1H), 3.30 (s, 3H). ${ }^{13} \mathbf{C}$ NMR (100 MHz, CDCl $\left.\mathbf{3}\right) \delta 175.76,145.84$, $142.01,141.31,130.59,130.11,129.53,128.72,128.24,124.85,123.48,108.89$, 93.91, 79.57, 26.90. HRMS (ESI) calculated $[\mathrm{M}-\mathrm{OH}]^{+}$for $\mathrm{C}_{15} \mathrm{H}_{11} \mathrm{INO}$ : 347.9880, found: 347.9884. FTIR $\left(\mathbf{c m}^{-1}\right)$ 3301, 2925, 1706, 1649, 1500, 1251, 1169, 928.

\section{2-Iodobenzoic acid (6a) ${ }^{8}$}<smiles>O=C(O)c1ccccc1I</smiles>

$6 a$

Following the procedure, treatment of 2-(trimethylsilyl)phenyl trifluoromethanesulfonate $2 \mathrm{a}(0.075 \mathrm{~g}, 61 \mu \mathrm{L}, 0.25 \mathrm{mmol})$ and $\mathrm{KI}(0.104 \mathrm{~g}, 0.625$ $\mathrm{mmol})$ in the presence of KF $(0.029 \mathrm{~g}, 0.5 \mathrm{mmol})$ and 18-crown-6 (0.132 $\mathrm{g}, 0.5$ $\mathrm{mmol})$ in THF $(1.0 \mathrm{~mL})$ under $\mathrm{CO}_{2}$ atmosphere at $25^{\circ} \mathrm{C}$ for $24 \mathrm{~h}$ followed by quenching the reaction mixture using cold water, and washing with dichloromethane followed by extraction with ethyl acetate afforded 2-iodobenzoic acid 6a as white solid (0.018 g, 29\% yield). $\boldsymbol{R}_{\mathbf{f}}($ Pet. ether $/$ EtOAc $=50 / 50): 0.31 ;{ }^{1} \mathbf{H}$ NMR (400 MHz, dMSO-d $) \delta 13.31(\mathrm{bs}, 1 \mathrm{H}), 7.98$ $\left(\mathrm{dd}, J_{1}=7.8 \mathrm{~Hz}, J_{2}=1.0 \mathrm{~Hz}, 1 \mathrm{H}\right), 7.71\left(\mathrm{dd}, J_{1}=7.7 \mathrm{~Hz}, J_{2}=1.6 \mathrm{~Hz}, 1 \mathrm{H}\right), 7.50-7.46(\mathrm{~m}, 1 \mathrm{H})$, 7.26-7.21 (m, 1H). ${ }^{13} \mathbf{C}$ NMR (100 MHz, dMSO-d $\left.\mathbf{6}\right) \delta 168.15,140.50,136.92,132.45,130.05$, 128.17, 94.11. FTIR (cm-1) 3752, 3585, 3424, 2697, 2365, 1650, 1219, 1021.

\section{Functionalization of 2-IodoBenzyl Alcohols}

\section{Using Sonogashira Coupling}

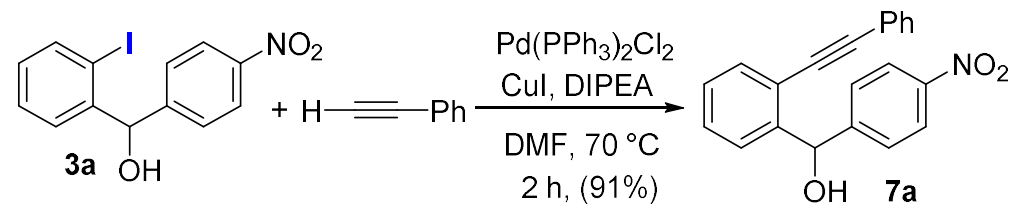

\footnotetext{
${ }^{8}$ Mukhopadhyay, S.; Batra, S. Chem. Eur. J., 2018, 24, 14622.
} 


\section{(4-nitrophenyl)(2-(phenylethynyl)phenyl)methanol (7a)}<smiles>O=[N+]([O-])c1ccc(C(O)c2ccccc2C#Cc2ccccc2)cc1</smiles>

Following the literature procedure, ${ }^{9}$ to a mixture of (2-iodophenyl)(4nitrophenyl)methanol 3a (107 mg, $0.3 \mathrm{mmol})$ in DMF (2 mL) were added $\mathrm{Pd}\left(\mathrm{PPh}_{3}\right)_{2} \mathrm{Cl}_{2}$ (4.2 mg, 0.02 equiv) and $\mathrm{CuI}(2.3 \mathrm{mg}, 0.04$ equiv) under nitrogen. After the reaction mixture was stirred for $5 \mathrm{~min}$. at $\mathrm{rt}, \mathrm{N}, \mathrm{N}$ diisopropylamine (121 mg, $168 \mu \mathrm{L}, 4$ equiv) was added by a syringe. The reaction mixture was then heated at $70{ }^{\circ} \mathrm{C}$ and phenylacetylene $(37 \mathrm{mg}, 40 \mu \mathrm{L}, 1.2$ equiv) was added. After stirring the reaction mixture for $2 \mathrm{~h}$ at $70{ }^{\circ} \mathrm{C}$, the reaction mixture was washed with saturated aq. $\mathrm{NH}_{4} \mathrm{Cl}$ and water, and then extracted with EtOAc, dried over anhydrous $\mathrm{Na}_{2} \mathrm{SO}_{4}$ and concentrated under vacuum. Subsequent purification via silica gel flash column chromatography (Pet. ether/EtOAc $=90 / 10)$ of the crude reaction mixture afforded (4-nitrophenyl)(2-(phenylethynyl)phenyl) methanol $7 \mathbf{a}$ as a yellow solid (90 $\mathrm{mg}, 91 \%$ yield).

$\boldsymbol{R}_{\mathbf{f}}($ Pet. ether $/$ EtOAc $=85 / 15): 0.40 ;{ }^{1} \mathbf{H}$ NMR (400 MHz, $\left.\mathbf{C D C l}_{3}\right) \delta 8.17(\mathrm{~d}, J=8.8 \mathrm{~Hz}, 2 \mathrm{H})$, $7.66(\mathrm{~d}, J=8.8 \mathrm{~Hz}, 2 \mathrm{H}), 7.57-7.55(\mathrm{~m}, 1 \mathrm{H}), 7.49-7.46(\mathrm{~m}, 3 \mathrm{H}), 7.39-7.35(\mathrm{~m}, 4 \mathrm{H}), 7.33-7.29$ (m, 1H), 6.48 (s, 1H), 2.79 (bs, 1H). ${ }^{13} \mathbf{C}$ NMR (100 MHz, CDCl 3 ) $\delta 150.38,147.27,144.42,132.83$, 131.57, 129.33, 129.00, 128.71, 128.26, 127.32, 126.65, 123.73, 122.64, 121.44, 95.15, 87.08, 73.27. HRMS (ESI) calculated $[\mathrm{M}-\mathrm{OH}]^{+}$for $\mathrm{C}_{21} \mathrm{H}_{14} \mathrm{NO}_{2}: 312.1019$, found: 312.1028 . FTIR $\left(\mathbf{c m}^{-1}\right)$ 3582, 1518, 1344, 1179, 1034, 825.

\section{Procedure for the Au-catalyzed cyclization of 7 a}

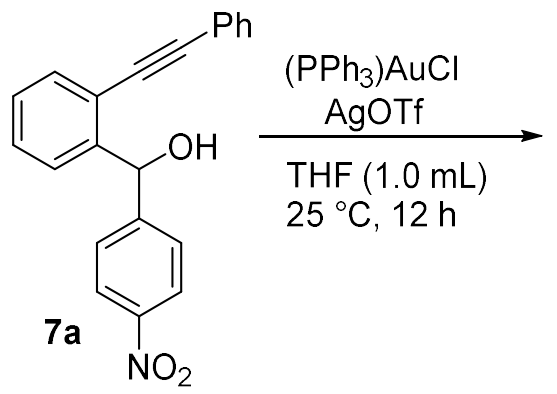<smiles></smiles><smiles></smiles>

To a flame-dried screw-capped test tube equipped with a magnetic stir bar was added the $\left(\mathrm{PPh}_{3}\right) \mathrm{AuCl}(2.5 \mathrm{mg}, 0.005 \mathrm{mmol})$ and $\operatorname{AgOTf}(1.3 \mathrm{mg}, 0.005 \mathrm{mmol})$ in a glove-box. Then (4nitrophenyl)(2-(phenylethynyl)phenyl)methanol $7 \mathbf{a}(33 \mathrm{mg}, 0.1 \mathrm{mmol})$ was added outside the glove box under nitrogen atmosphere followed by addition of THF (1 mL). The resulting

${ }^{9}$ Li, D. Y.; Shi, K. J.; Mao, X. F.; Zhao, Z. L.; Wu, X. Y.; Liu, P. N. Tetrahedron 2014, 70, 7022. 
solution was stirred at $\mathrm{rt}$ for $12 \mathrm{~h}$. After $12 \mathrm{~h}$, the solvent was evaporated and the crude residue pre-adsorbed on silica gel and purified by flash column chromatography (Pet. ether /EtOAc $=$ 95/5) afforded (Z)-1-benzylidene-3-(4-nitrophenyl)-1,3-dihydroisobenzofuran $\mathbf{8 a}$ as major isomer (27 $\mathrm{mg}, \quad 82 \%$ yield) along with (Z)-1-benzylidene-3-(4-nitrophenyl)-1,3dihydroisobenzofuran $\mathbf{8 b}$ ( $5 \mathrm{mg}, 15 \%$ yield).

\section{(Z)-1-benzylidene-3-(4-nitrophenyl)-1,3-dihydroisobenzofuran 8a}

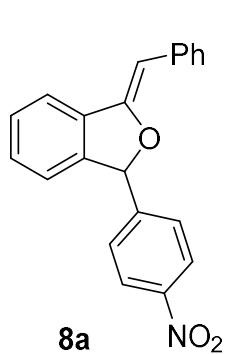

Yellow solid (27 mg, 82\% yield), $\boldsymbol{R}_{\mathbf{f}}($ Pet. ether $/$ EtOAc $=85 / 15): 0.50 ;{ }^{1} \mathbf{H}$ NMR (400 MHz, CDCl $\left.\mathbf{~}_{3}\right) \delta 8.23(\mathrm{~d}, J=8.6 \mathrm{~Hz}, 2 \mathrm{H}), 7.79$ (d, $\left.J=8.8 \mathrm{~Hz}, 2 \mathrm{H}\right), 7.64$ (d, $J=7.7 \mathrm{~Hz}, 1 \mathrm{H}), 7.55(\mathrm{~d}, J=8.6 \mathrm{~Hz}, 2 \mathrm{H}), 7.44-7.32(\mathrm{~m}, 4 \mathrm{H}), 7.21-7.16(\mathrm{~m}, 2 \mathrm{H})$, $6.66(\mathrm{~s}, 1 \mathrm{H}), 6.08$ (s, 1H). ${ }^{13} \mathrm{C}$ NMR (100 MHz, $\left.\mathbf{C D C l}_{3}\right) \delta$ 154.93, 148.08, $147.21,141.22,135.92,134.33,129.35,129.10,128.58,128.22,127.19,125.95$, 124.24, 122.07, 120.31, 97.60, 86.13. HRMS (ESI) calculated $[\mathrm{M}+\mathrm{H}]{ }^{+}$for $\mathrm{C}_{21} \mathrm{H}_{16} \mathrm{NO}_{3}: 330.1130$, found: 330.1125. FTIR (cm-1) 3052, 1659, 1523, 1349, 1107, 1038, 852.

\section{(Z)-1-benzylidene-3-(4-nitrophenyl)-1,3-dihydroisobenzofuran 8b}

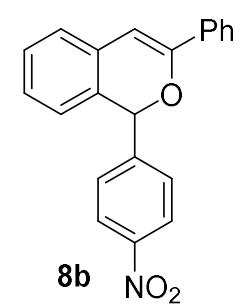

Yellow solid (5 mg, 15\% yield), $\boldsymbol{R}_{\mathbf{f}}($ Pet. ether $/$ EtOAc $=90 / 10): 0.60 ;{ }^{1} \mathbf{H}$ NMR (400 MHz, $\left.\mathbf{C D C l}_{3}\right) \delta 8.20(\mathrm{~d}, J=8.6 \mathrm{~Hz}, 2 \mathrm{H}), 7.75-7.73(\mathrm{~m}, 2 \mathrm{H}), 7.57(\mathrm{~d}, J=$ 8.6 Hz, 2H), 7.42-7.32 (m, 4H), 7.24-7.19 (m, 2H), 6.87 (d, J=7.7 Hz, 1H), 6.53 (s, 1H), 6.37 (s, 1H). ${ }^{13} \mathbf{C}$ NMR (100 MHz, CDCl $) \delta 152.14,147.95,147.18$, $133.94,131.48,129.28,129.05,129.00,128.60,128.58,126.99,125.26,125.08$, 124.41, 123.72, 101.41, 78.89. HRMS (ESI) calculated $[\mathrm{M}+\mathrm{H}]^{+}$for $\mathrm{C}_{21} \mathrm{H}_{16} \mathrm{NO}_{3}: 330.1130$, found: 330.1125. FTIR (cm $\left.{ }^{-1}\right)$ 3072, 3061, 2365, 1521, 1346, 1219, 1062, 855.

\section{Using Heck Coupling:}<smiles>O=[N+]([O-])c1ccc(/C=C/c2cc(C(O)c3ccccc3I)ccc2C(O)c2ccc([N+](=O)[O-])cc2)cc1</smiles> 


\section{(E)-(4-Nitrophenyl)(2-styrylphenyl)methanol (9a)}<smiles>O=[N+]([O-])c1ccc(C(O)c2ccccc2/C=C/c2ccccc2)cc1</smiles>

Following the literature procedure, ${ }^{10}$ a mixture of (2-iodophenyl)(4nitrophenyl)methanol 3a (44 mg, $0.125 \mathrm{mmol}$ ), styrene (26 mg, $29 \mu \mathrm{L}$, $0.25 \mathrm{mmol})$, palladium acetate $(2.8 \mathrm{mg}, 0.0125 \mathrm{mmol})$, tri-o-tolylphosphine

$(7.6 \mathrm{mg}, 0.025 \mathrm{mmol})$ and triethylamine $(51 \mathrm{mg}, 70 \mu \mathrm{L}, 0.5 \mathrm{mmol})$, in acetonitrile $(1.25 \mathrm{ml})$ was heated at $80{ }^{\circ} \mathrm{C}$ for $12 \mathrm{~h}$. The reaction mixture was concentrated under reduced pressure and then purified by flash column chromatography to afford (E)-(4nitrophenyl) (2-styrylphenyl)methanol 9a as a yellow oil (35 $\mathrm{mg}, 84 \%$ ).

$\boldsymbol{R}_{\mathbf{f}}($ Pet. ether $/$ EtOAc $=85 / 15): 0.39 ;{ }^{1} \mathbf{H}$ NMR (400 MHz, $\left.\mathbf{C D C l}_{3}\right) 8.17(\mathrm{~d}, J=8.8 \mathrm{~Hz}, 2 \mathrm{H}), 7.64$ $(\mathrm{d}, J=7.6 \mathrm{~Hz}, 1 \mathrm{H}), 7.56(\mathrm{~d}, J=8.6 \mathrm{~Hz}, 2 \mathrm{H}), 7.44-7.27(\mathrm{~m}, 9 \mathrm{H}), 6.95(\mathrm{~d}, J=16 \mathrm{~Hz}, 1 \mathrm{H}), 6.26$ (s, 1H), $2.53(\mathrm{~s}, 1 \mathrm{H}) .{ }^{13} \mathbf{C}$ NMR (100 MHz, $\left.\mathbf{C D C l}_{3}\right) \delta$ 150.53, 147.21, 139.77, 137.10, 136.32, $132.22,128.92,128.22,127.85,127.38,127.00,126.72,125.33,123.74,72.86$. HRMS (ESI) calculated $[\mathrm{M}-\mathrm{OH}]^{+}$for $\mathrm{C}_{21} \mathrm{H}_{16} \mathrm{NO}_{2}: 314.1176$, found: 314.1180. FTIR (cm $\left.{ }^{-1}\right)$ 3583, 3025, 2364, $1600,1518,1344,1027,962,850$.

\section{Using Suzuki Coupling}

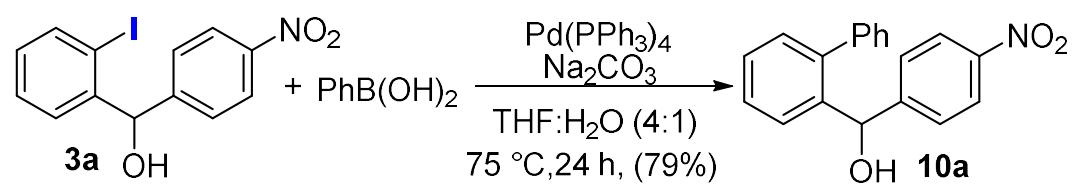

\section{[1,1'-biphenyl]-2-yl(4-nitrophenyl)methanol (10a)}<smiles>O=[N+]([O-])c1ccc(C(O)c2ccccc2-c2ccccc2)cc1</smiles>

Following the literature procedure, ${ }^{11}$ (2-iodophenyl)(4-nitrophenyl) methanol 3a (44 mg, $0.125 \mathrm{mmol})$, phenylboronic acid (23 $\mathrm{mg}, 1.5$ equiv), $\mathrm{Pd}\left(\mathrm{PPh}_{3}\right)_{4}$ (4.3 mg, 0.03equiv), $\mathrm{Na}_{2} \mathrm{CO}_{3}$ (40 mg, 3 equiv) was added into a flame-dried screw-capped test tube equipped with a magnetic stir bar.The screw-capped test tubewas evacuated under vacuum and flushed with dry nitrogen three times and then $2 \mathrm{~mL}$ THF and $0.5 \mathrm{~mL}$ water was added. The reaction mixture was refluxed for $24 \mathrm{~h}$. After cooling to room temperature, the mixture was poured into water and

\footnotetext{
${ }^{10}$ Hirano, K.; Biju, A. T.; Piel, I.; Glorius, F. J. Am. Chem. Soc. 2009, 131, 14190.

${ }^{11}$ Chen, L.; Wang, Y.-H.; He, B.; Nie, H.; Hu, R.; Huang, F.; Qin, A.; Zhou, X.-S.; Zhao, Z.; Tang, B. Z. Angew. Chem., Int. Ed. 2015, 54, 4231.
} 
extracted with dichloromethane three times. The combined organic layers were dried over anhydrous $\mathrm{Na}_{2} \mathrm{SO}_{4}$. After filtration and solvent evaporation, a silica gel flash column chromatography (Pet. ether $/$ EtOAc $=90 / 10)$ of the crude reaction mixture afforded $\left[1,1^{\prime}-\right.$ biphenyl]-2-yl(4-nitrophenyl)methanol 10a as a yellow liquid (30 $\mathrm{mg}, 79 \%$ yield).

$\boldsymbol{R}_{\mathbf{f}}\left(\right.$ Pet. ether $/$ EtOAc $=$ 85/15): 0.40; ${ }^{1} \mathbf{H}$ NMR (400 $\left.\mathbf{M H z}, \mathbf{C D C l}_{3}\right) \delta 8.10-8.06(\mathrm{~m}, 2 \mathrm{H}), 7.43-$ 7.35 (m, 6H), 7.34-7.25 (m, 5H), $6.02(\mathrm{~s}, 1 \mathrm{H}), 2.33$ (bs, 1H). ${ }^{13} \mathbf{C}$ NMR (100 MHz, CDCl $) \delta$ 151.20, 147.07, 141.61, 140.50, 140.16, 130.47, 129.38, 128.58, 128.41, 128.25, 127.70, 127.35, 123.53, 71.84. HRMS (ESI) calculated $[\mathrm{M}-\mathrm{OH}]^{+}$for $\mathrm{C}_{19} \mathrm{H}_{14} \mathrm{NO}_{2}: 288.1019$, found: 288.1028 . FTIR (cm $\left.{ }^{-1}\right)$ 3418, 3061, 2363, 1601, 1519, 1345, 1011, 839. 
10. ${ }^{1} \mathrm{H}$ and ${ }^{13} \mathrm{C}$ NMR Spectra of Products

(2-Iodophenyl)(4-nitrophenyl)methanol (3a)
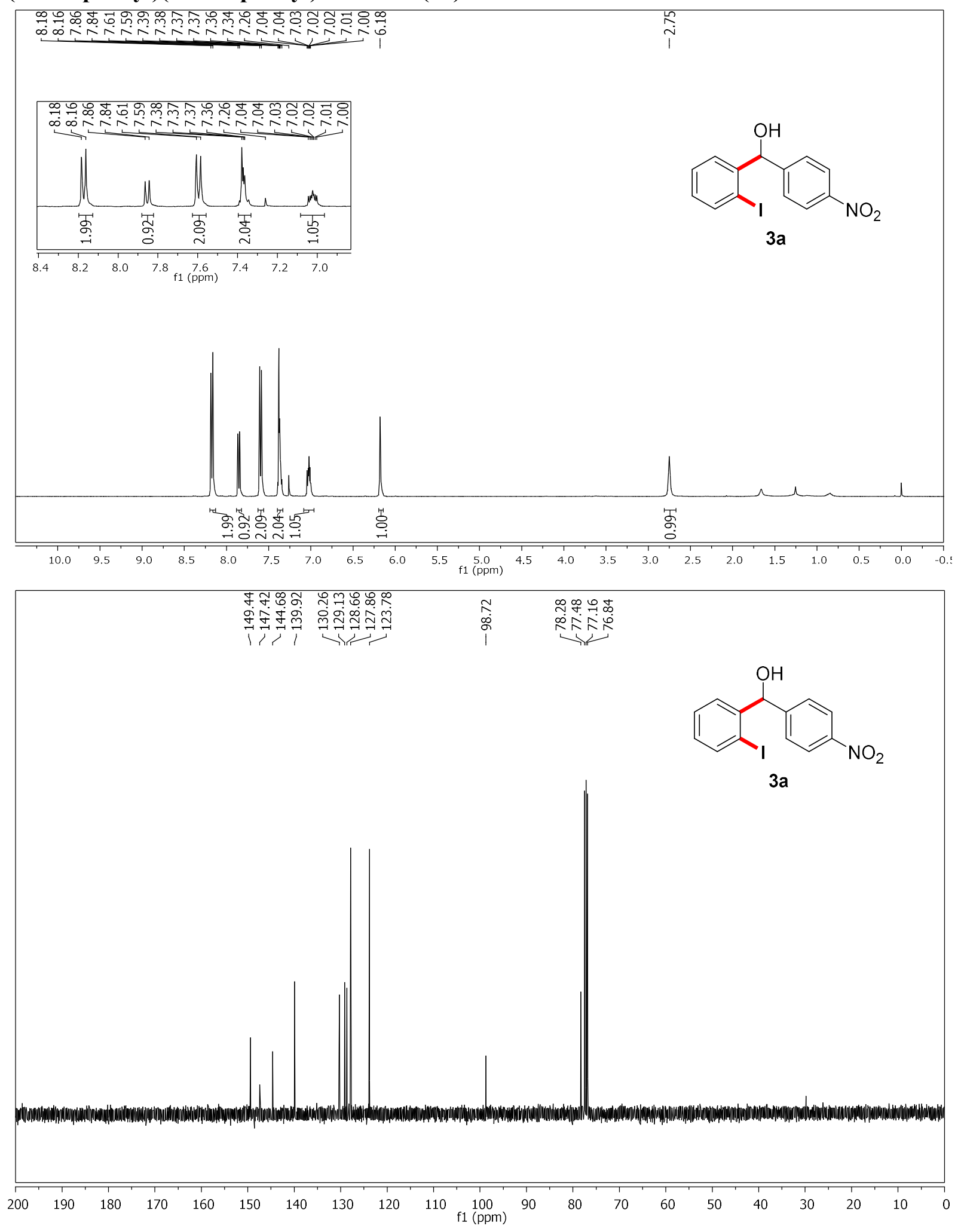

S40 
(2-Iodophenyl)(phenyl)methanol (3b) ${ }^{1}$
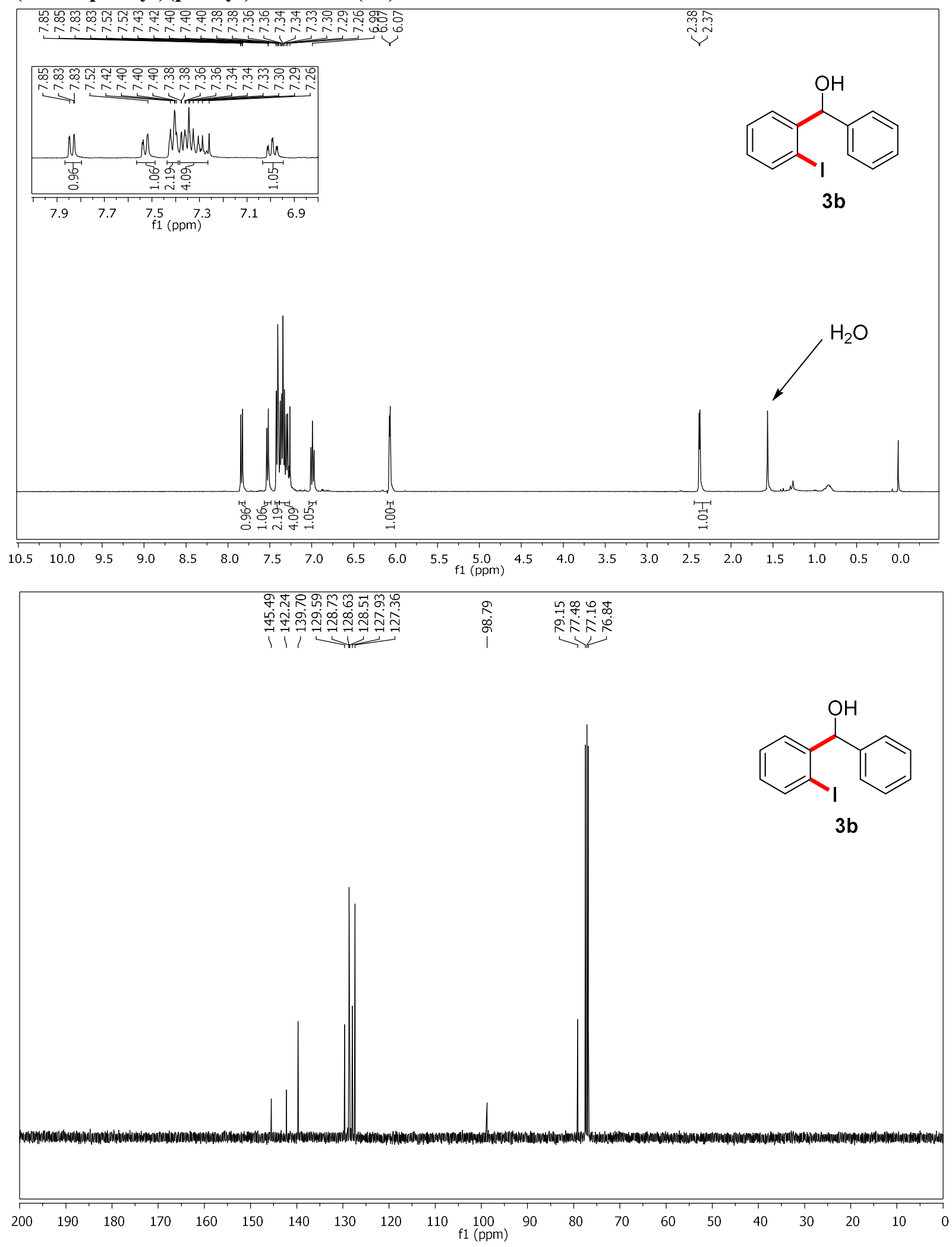
(4-Bromophenyl)(2-iodophenyl)methanol (3c)
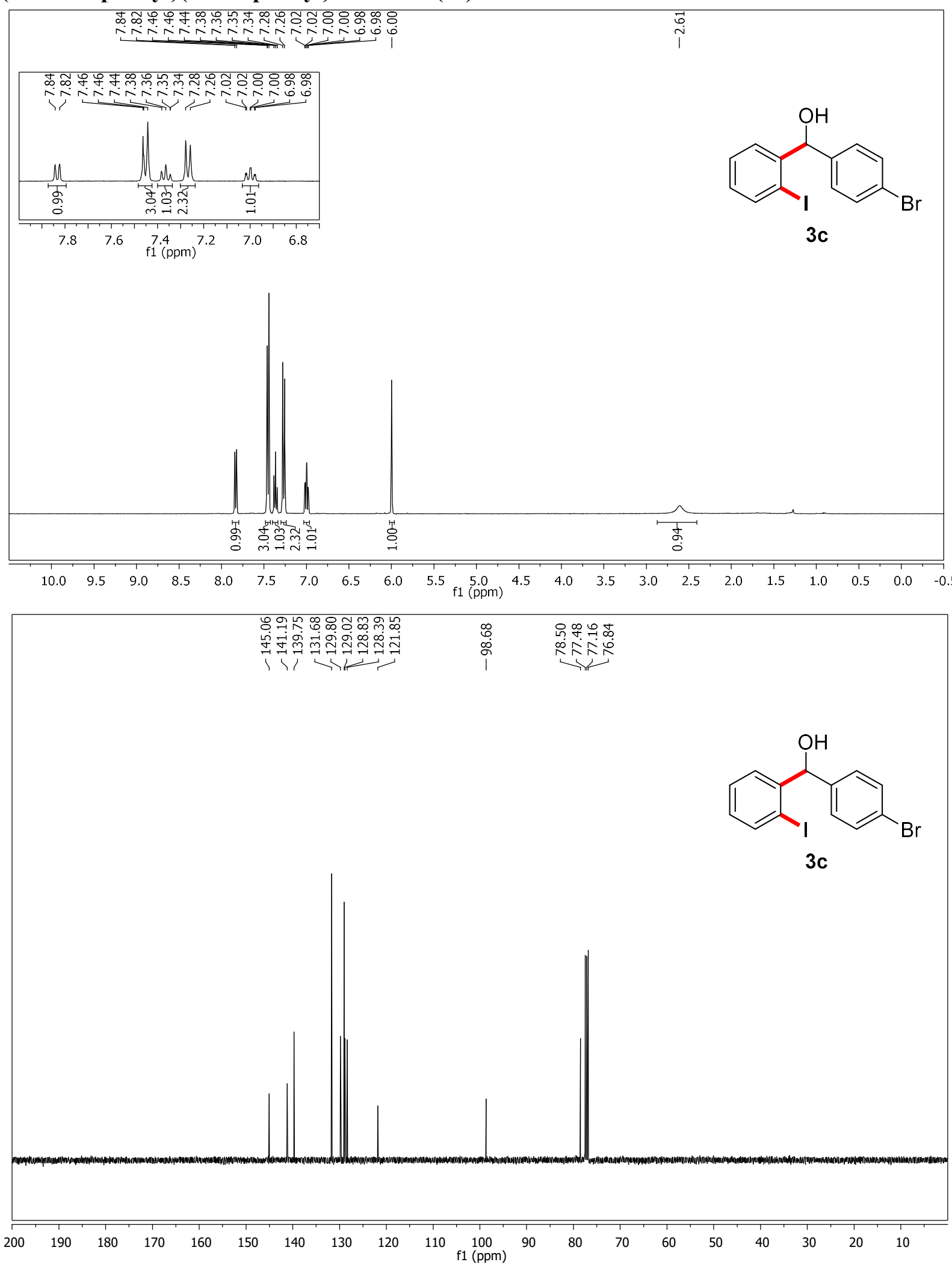
(4-Chlorophenyl)(2-iodophenyl)methanol (3d)
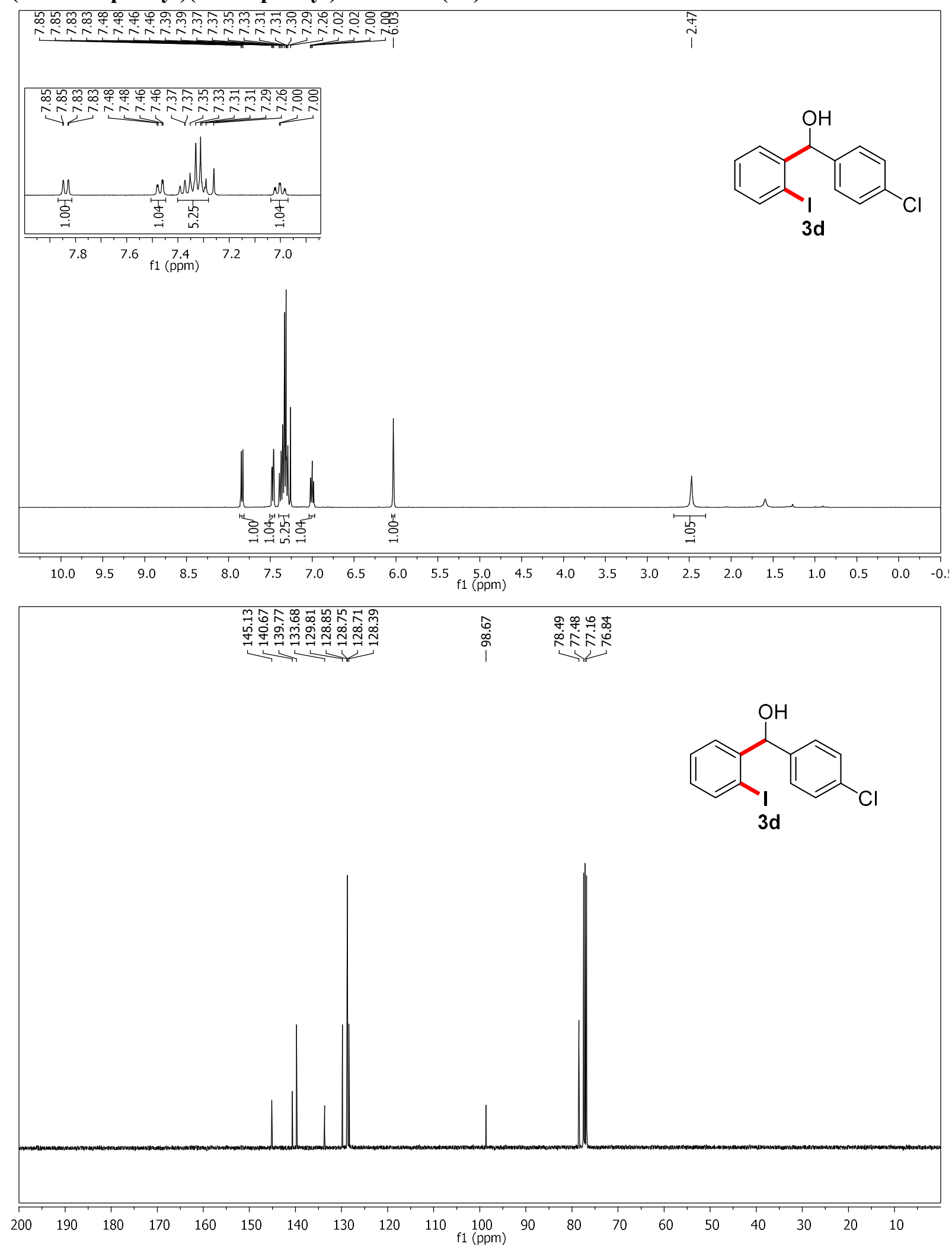
(4-Fluorophenyl)(2-iodophenyl)methanol (3e)
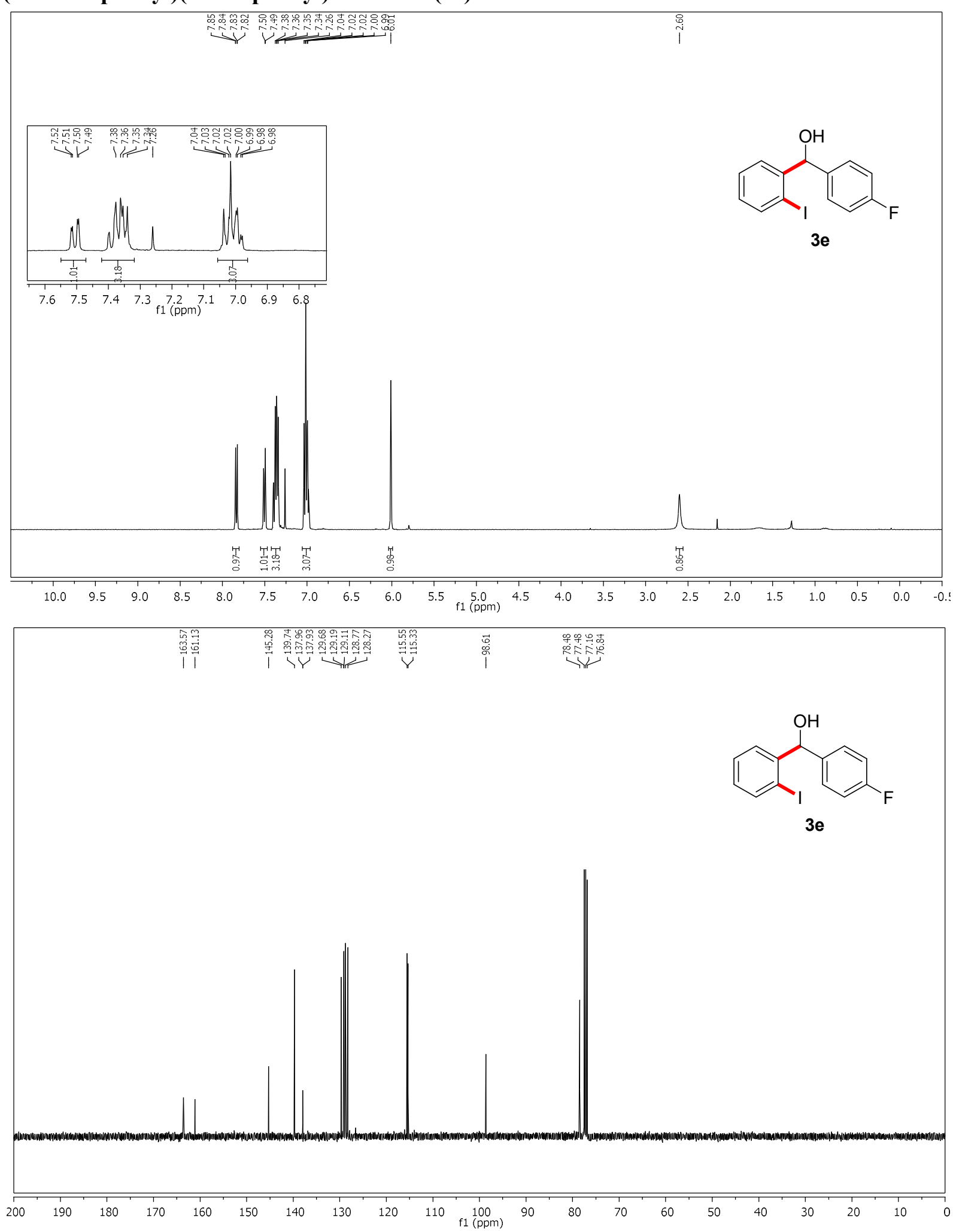

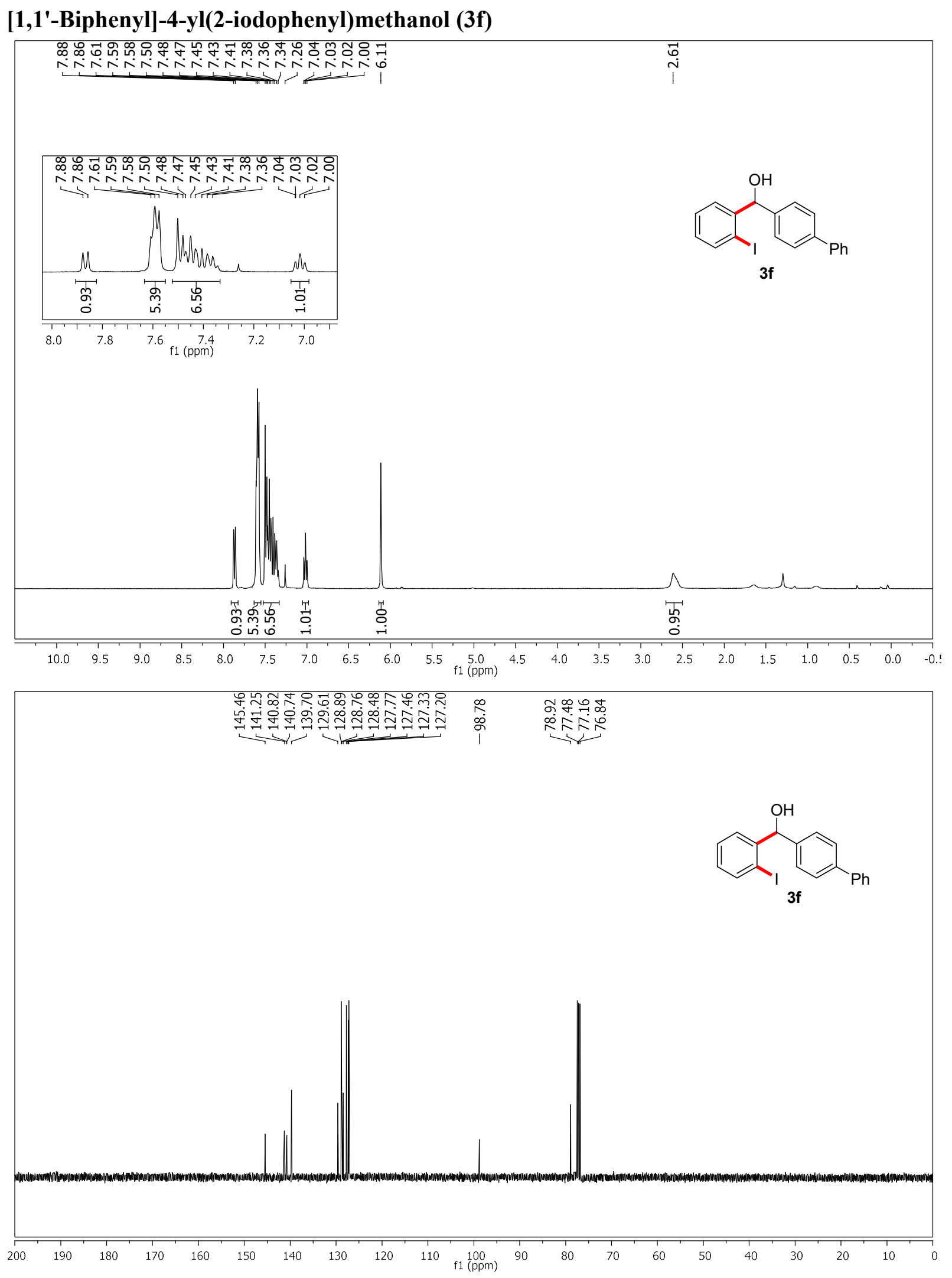

S45 
(2-Iodophenyl)(4-(trifluoromethyl)phenyl)methanol (3g)
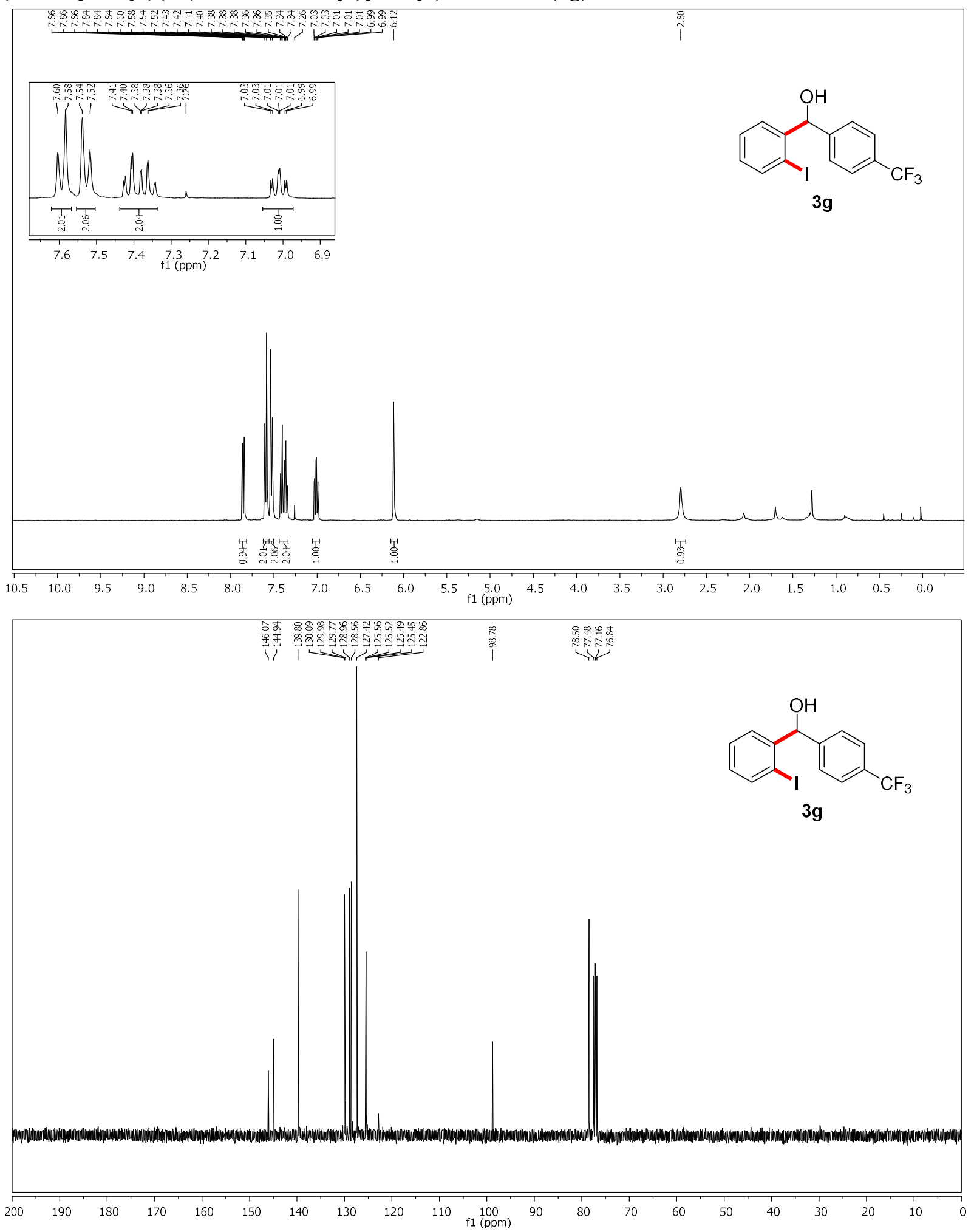

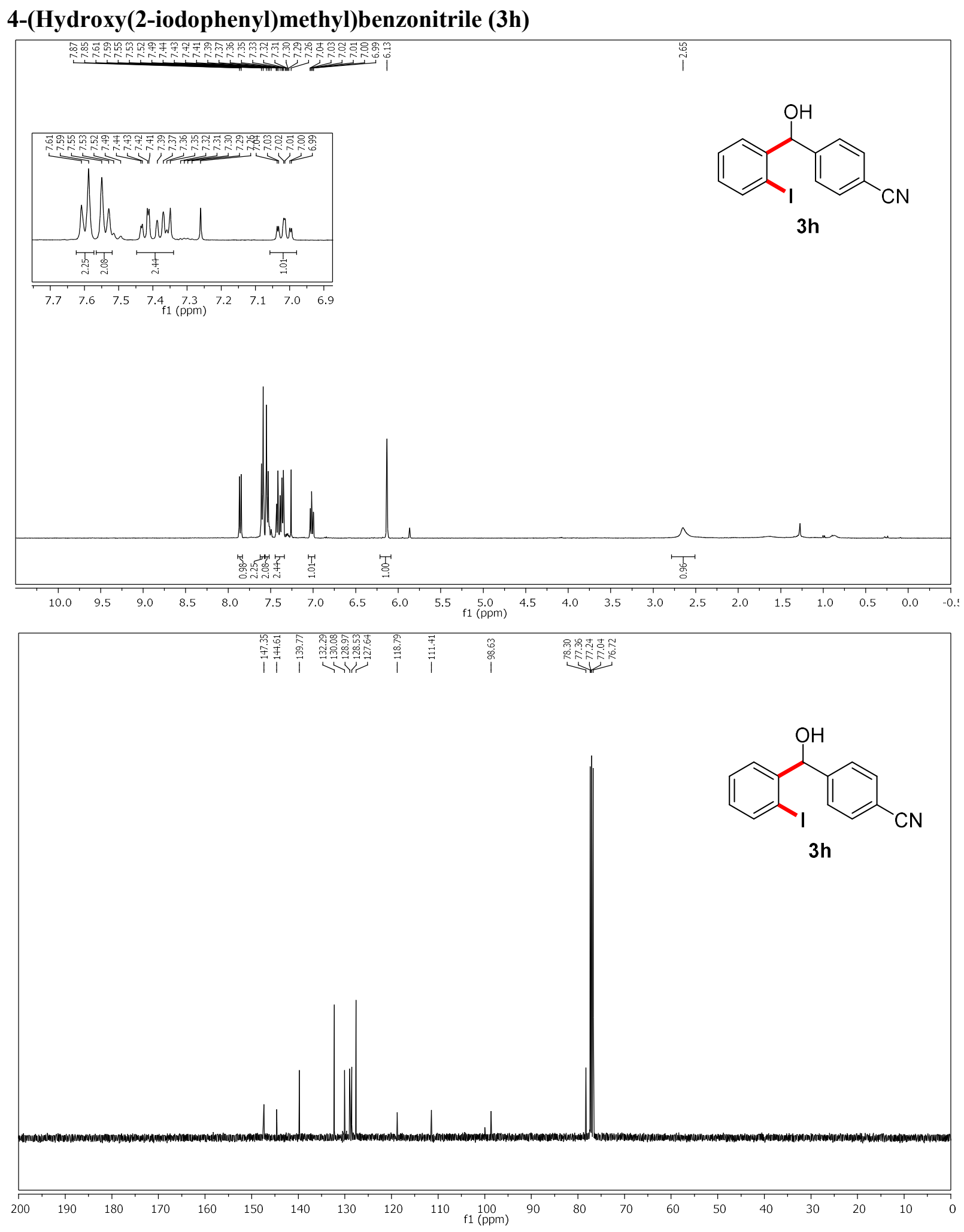

S47 

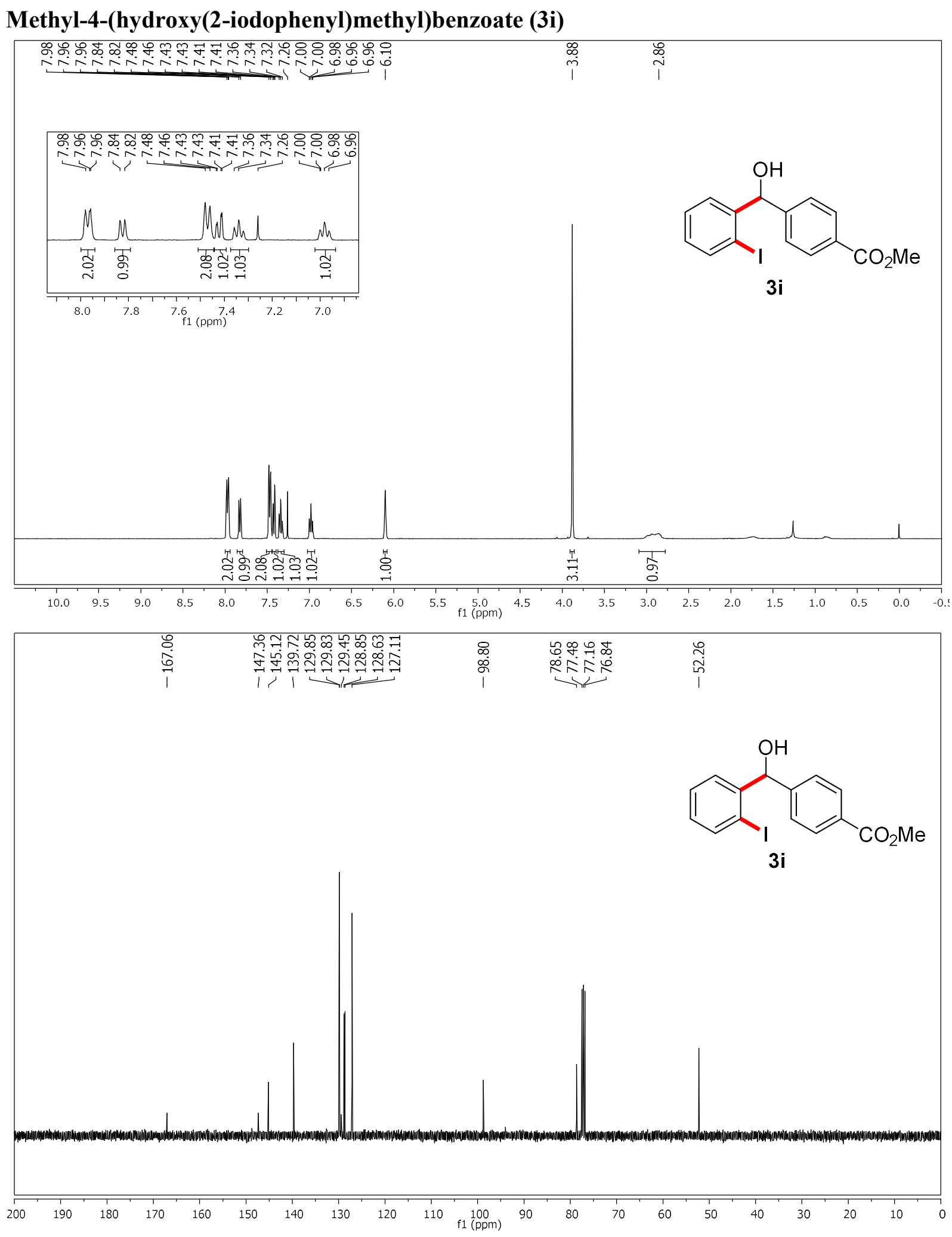

S48 

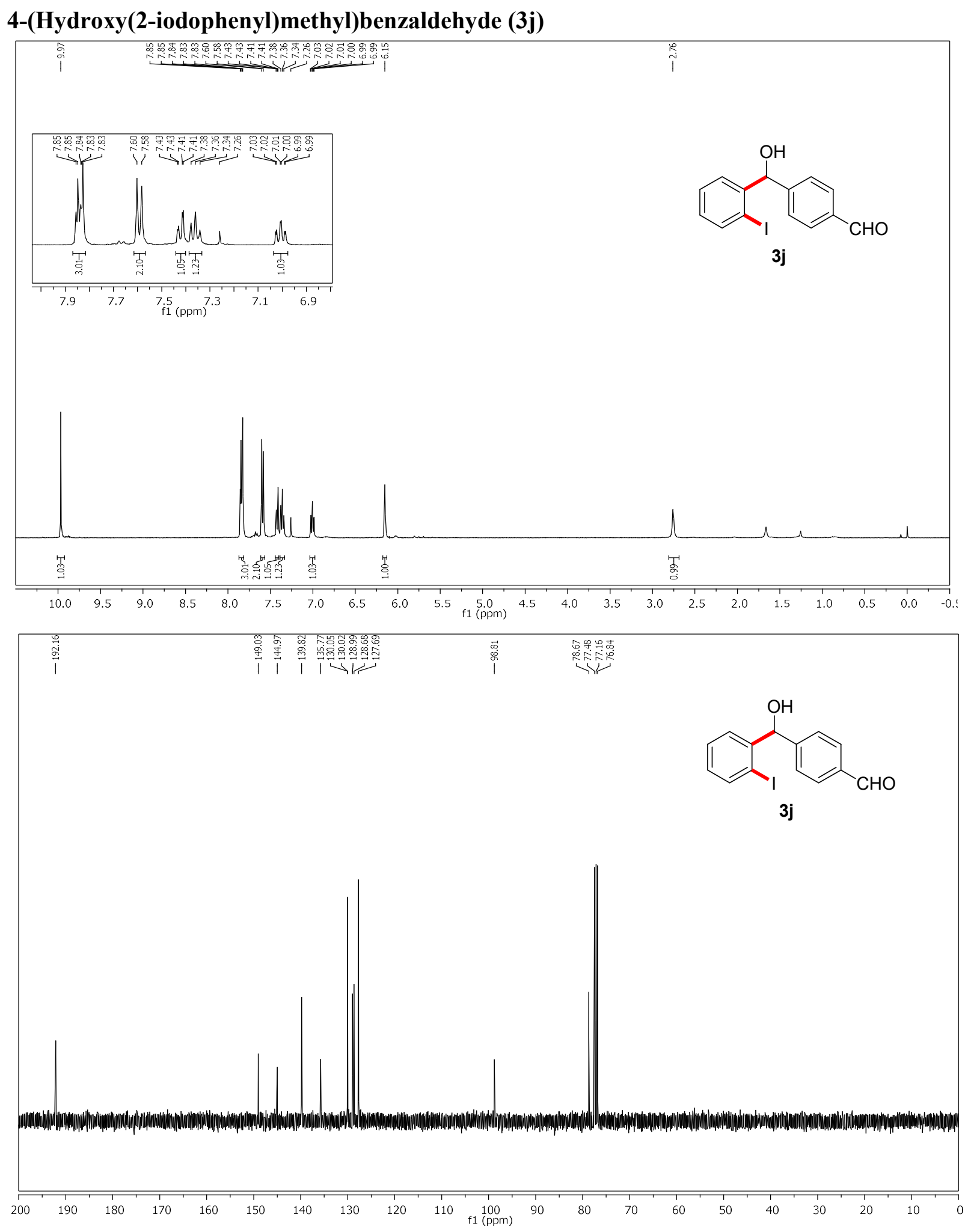

S49 
(3-Bromophenyl)(2-iodophenyl)methanol (3k)
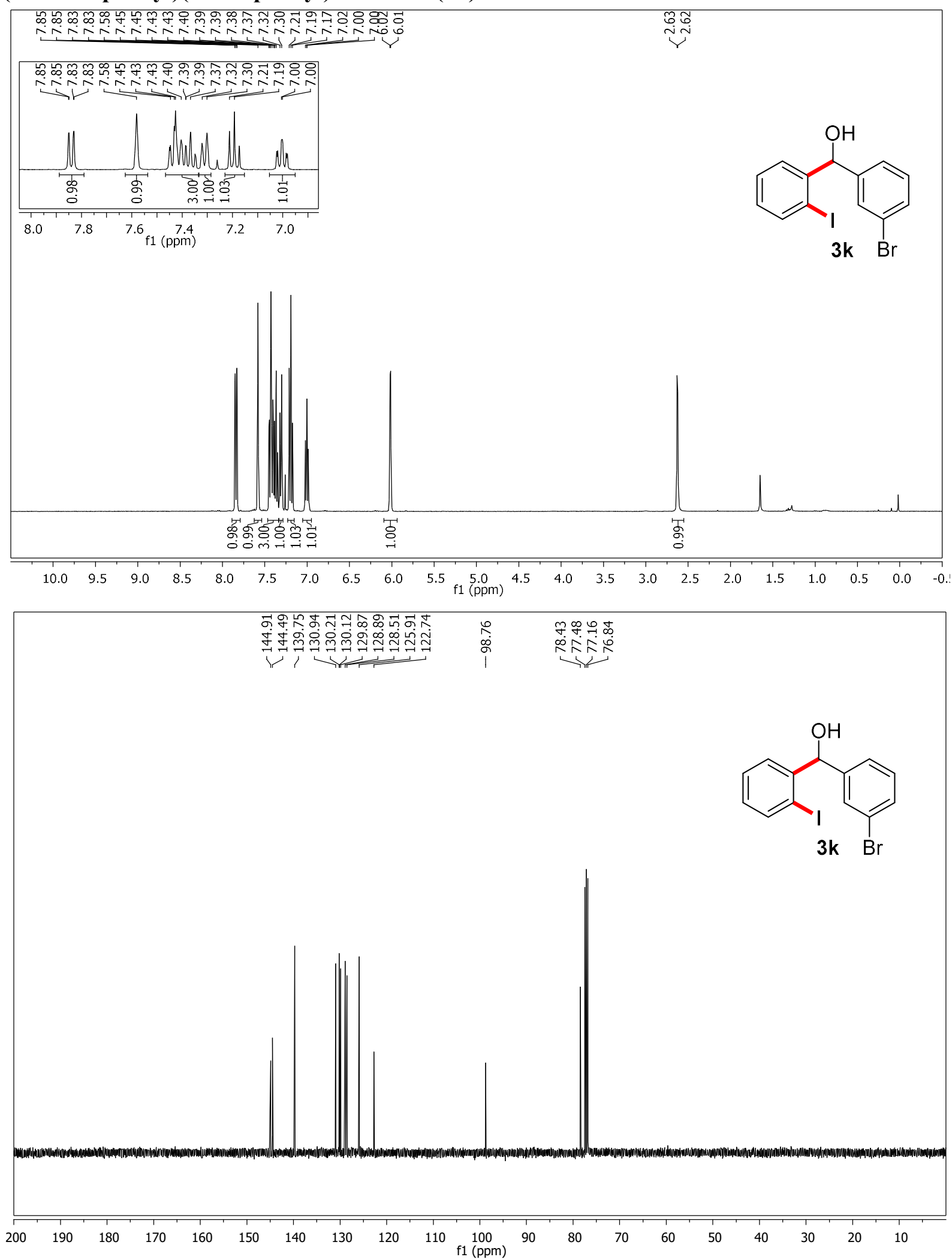


\section{(2-Fluorophenyl)(2-iodophenyl)methanol (3I)}
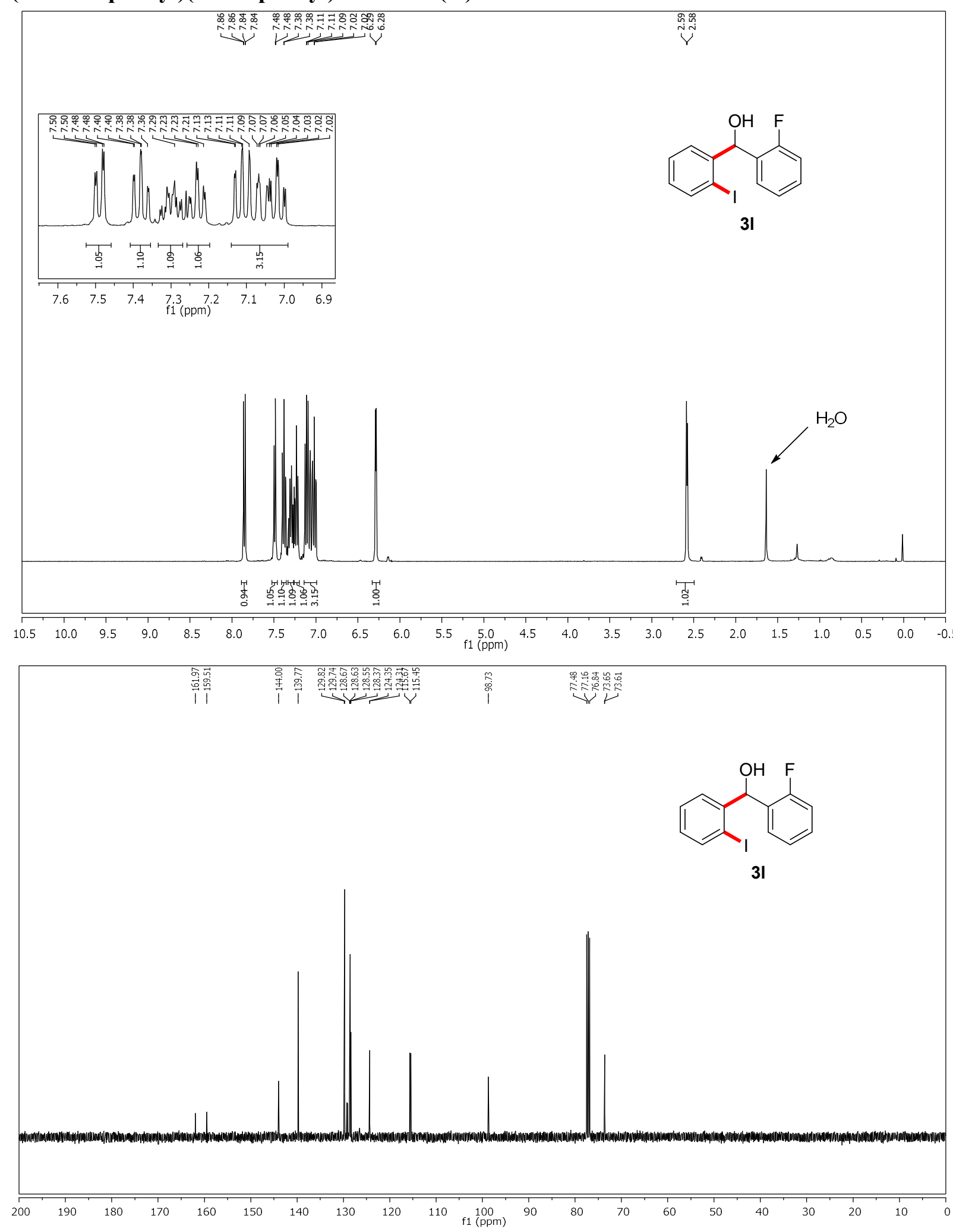
(2-Chlorophenyl)(2-iodophenyl)methanol (3m)
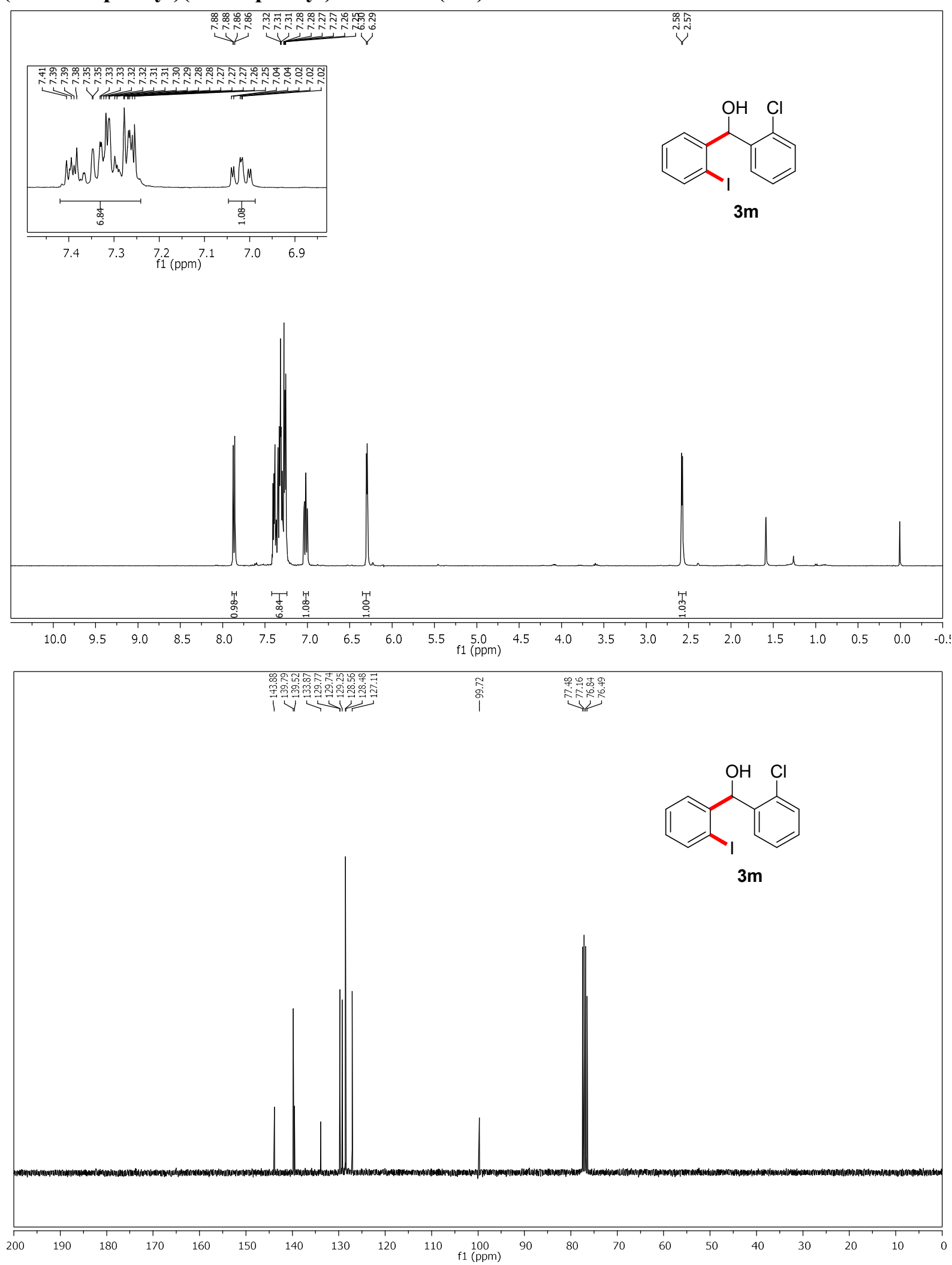
(2-Iodophenyl)(2-nitrophenyl)methanol (3n)
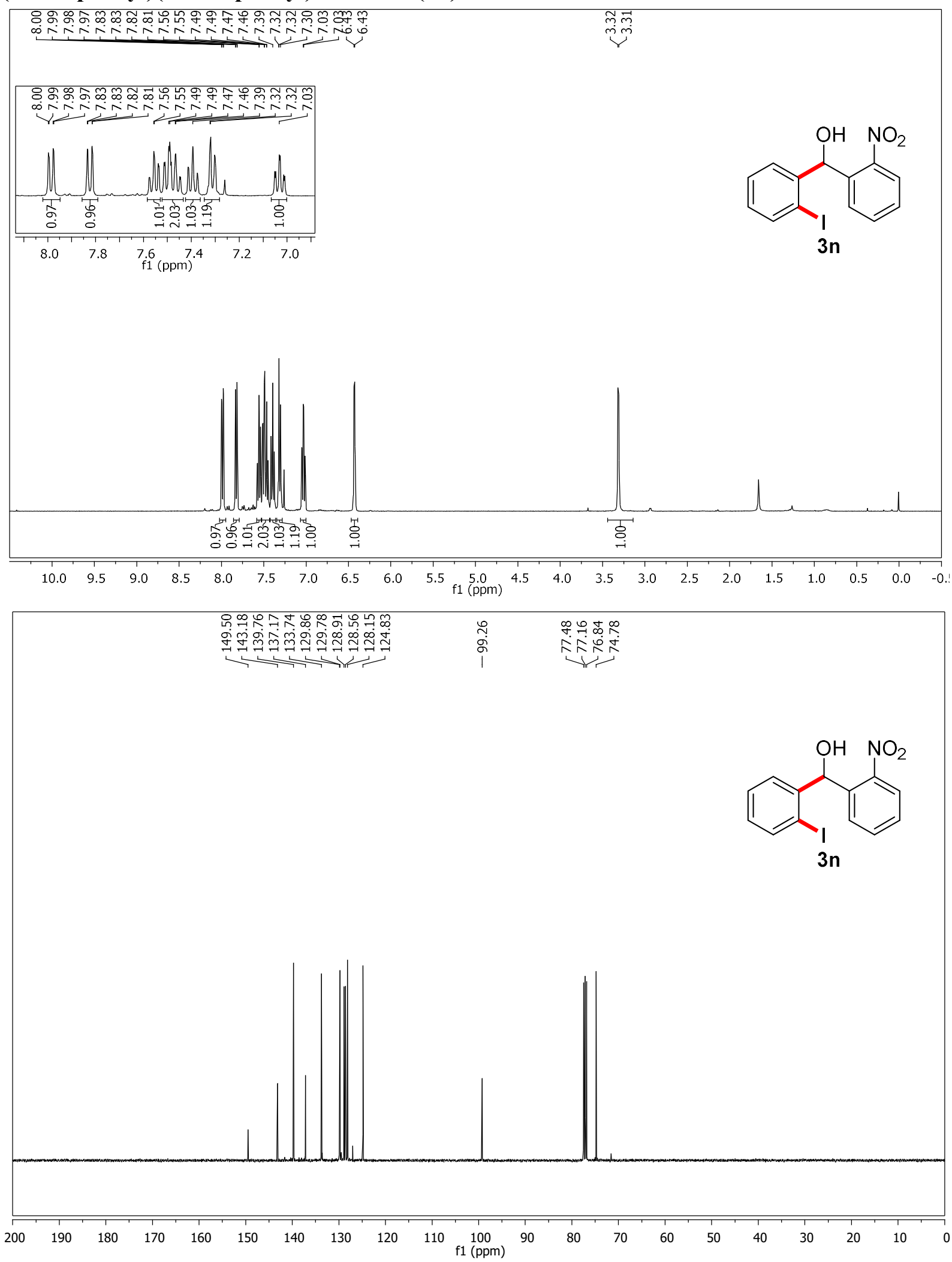


\section{(3,4-Dichlorophenyl)(2-iodophenyl)methanol (3o)}
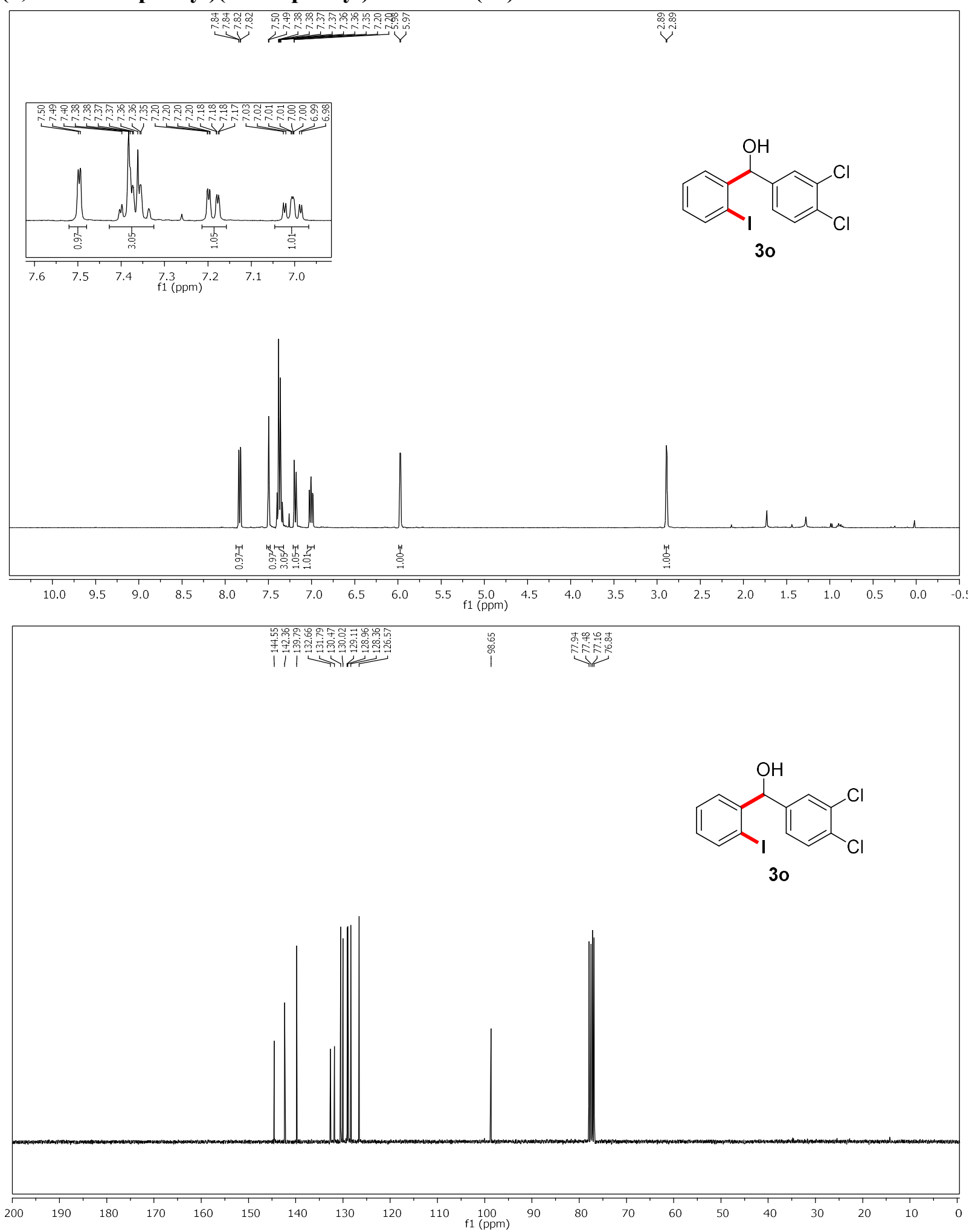
(2-Chloro-6-fluorophenyl)(2-iodophenyl)methanol (3p)

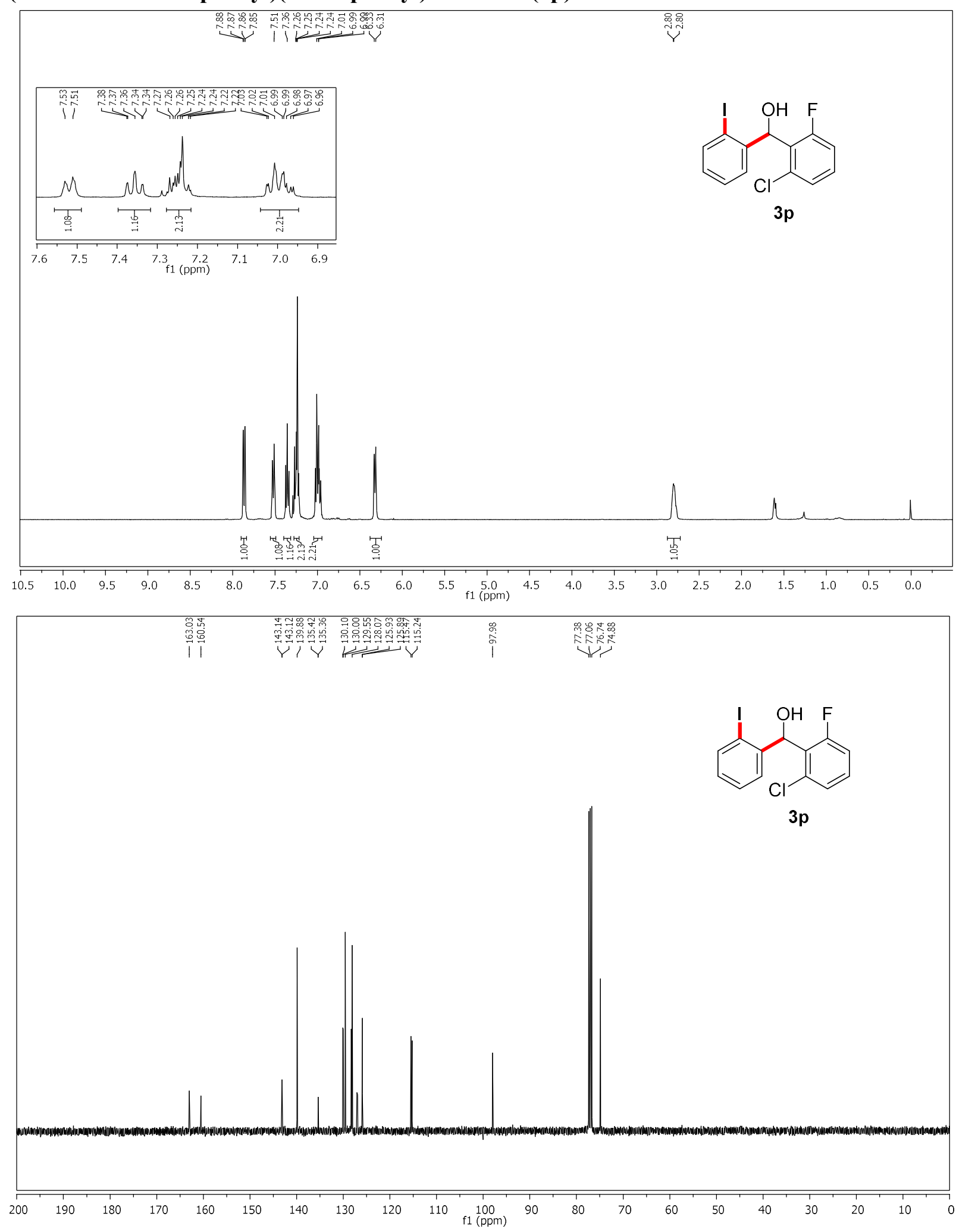




\section{(3,5-Bis(trifluoromethyl)phenyl)(2-iodophenyl)methanol (3q)}
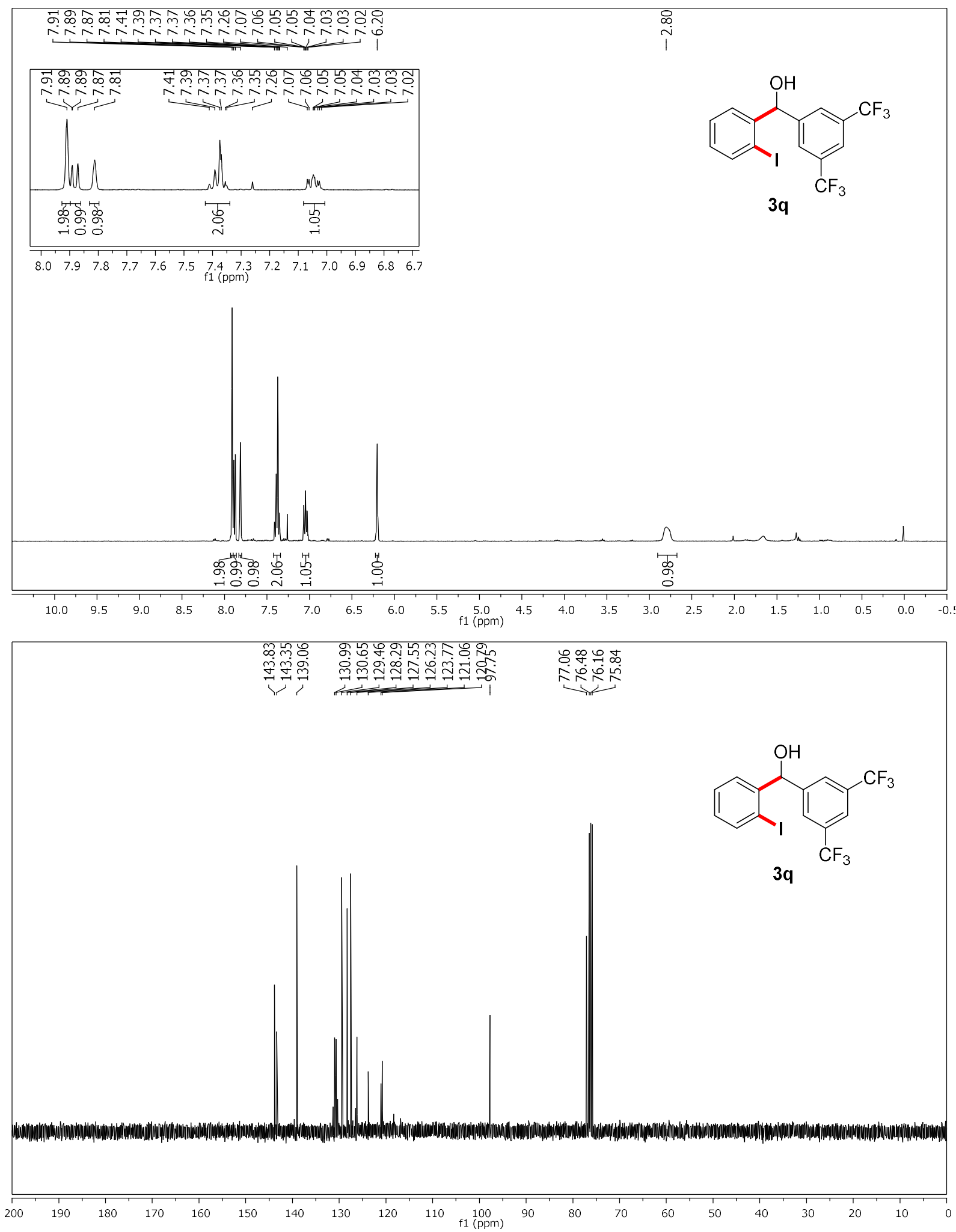
(2-Iodophenyl)(naphthalen-2-yl)methanol (3r)
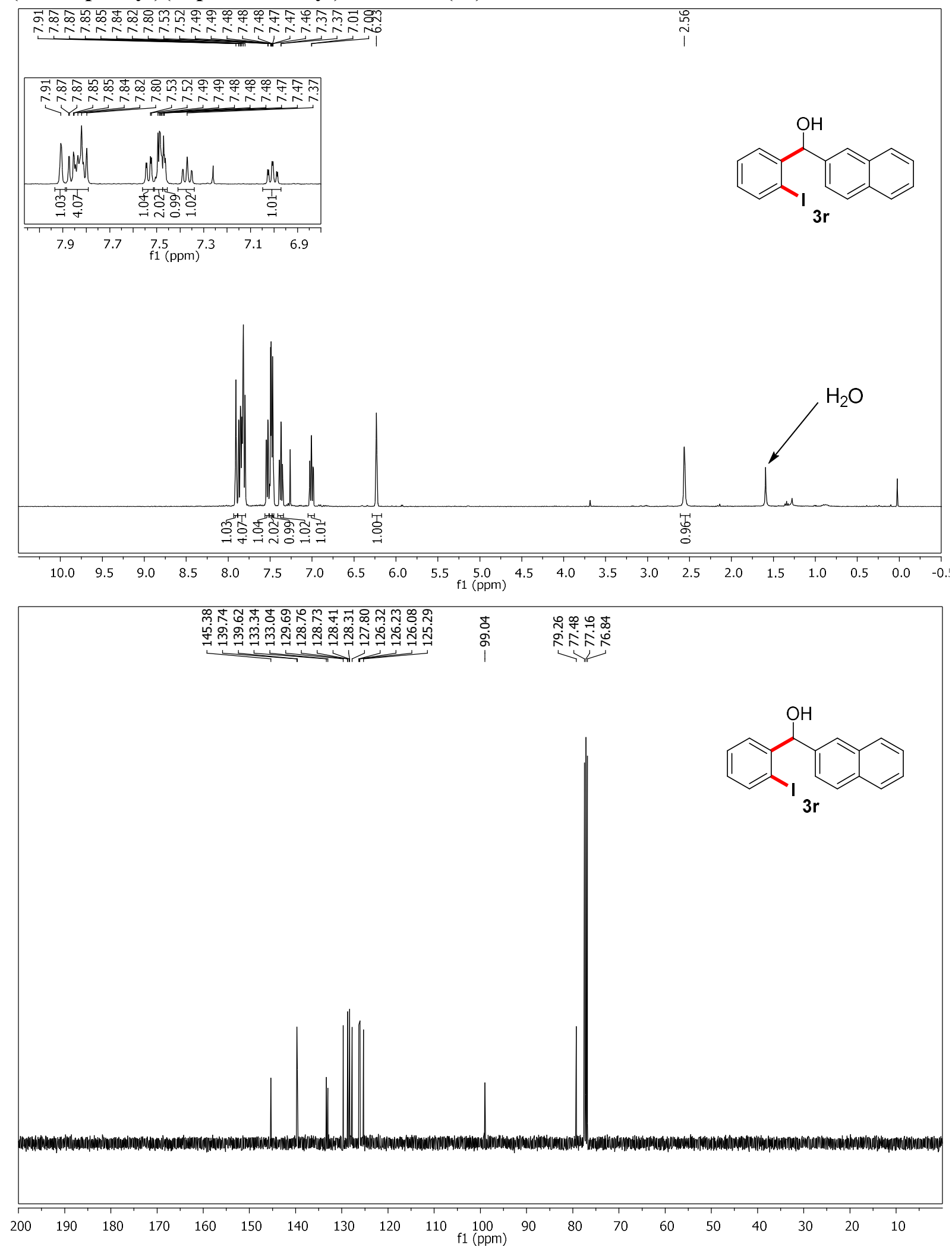


\section{(2-Iodophenyl)(pyren-1-yl)methanol (3s)}
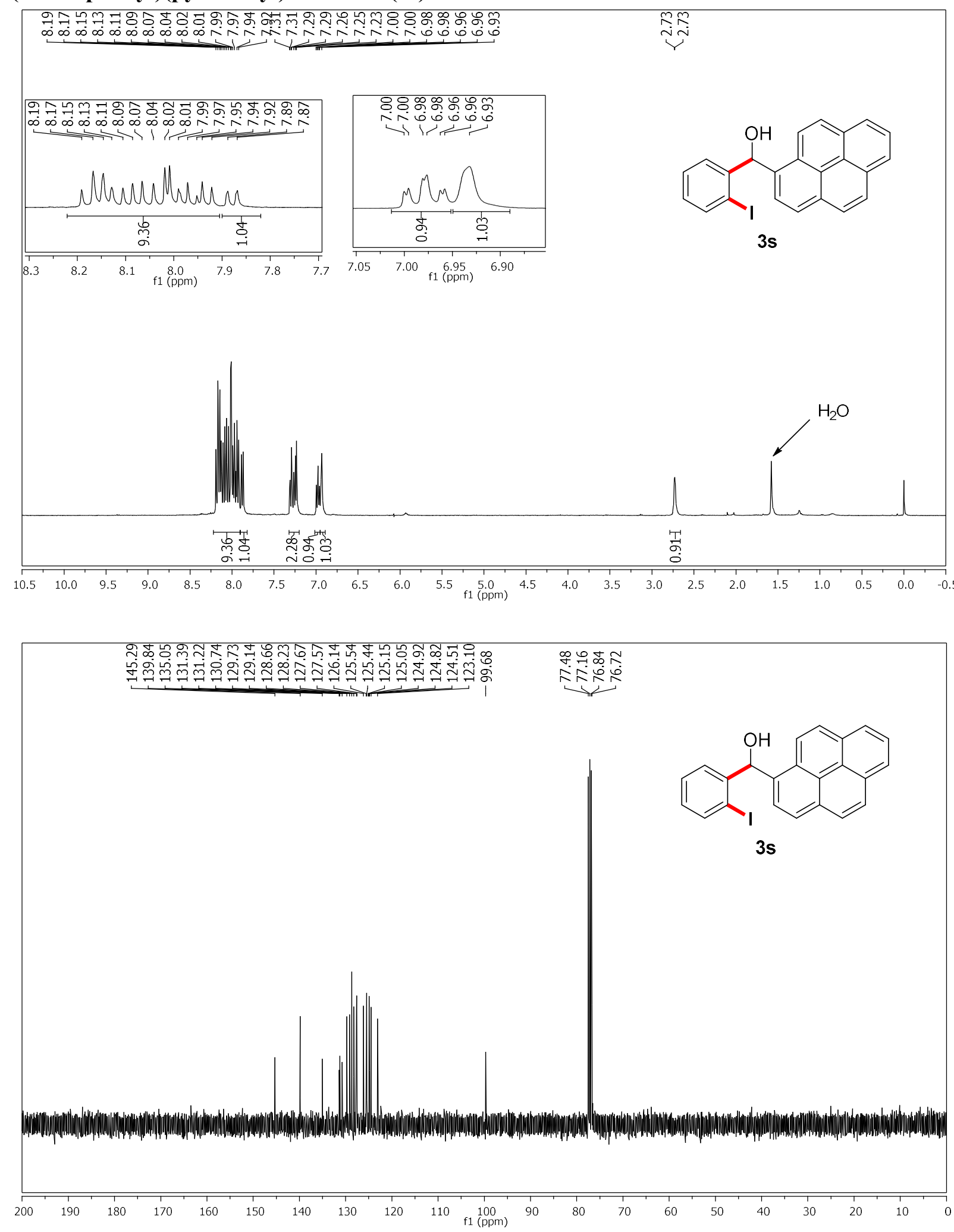
(2-Iodophenyl)(thiophen-2-yl)methanol (3t)
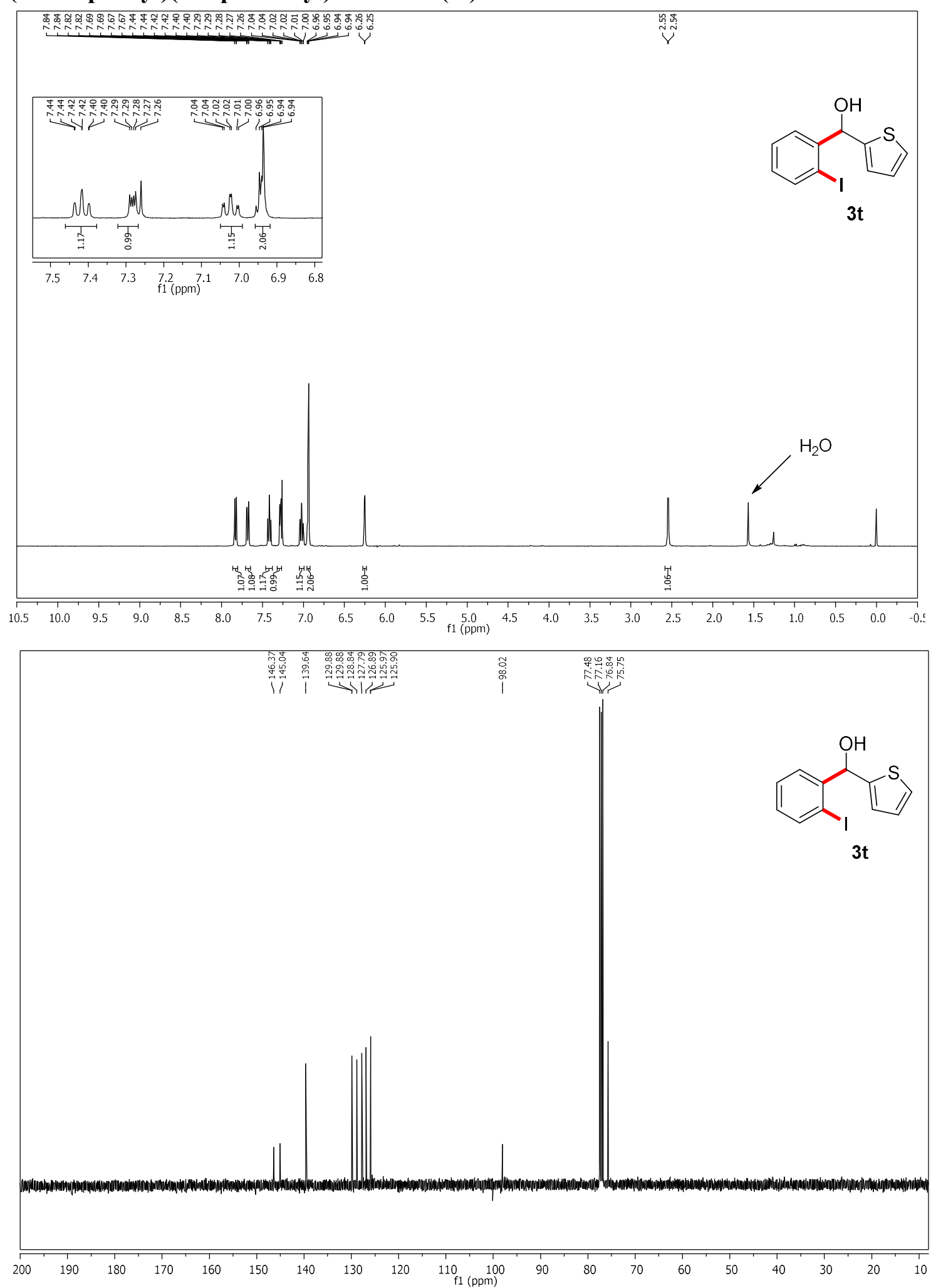
Benzofuran-2-yl(2-iodophenyl)methanol (3u)
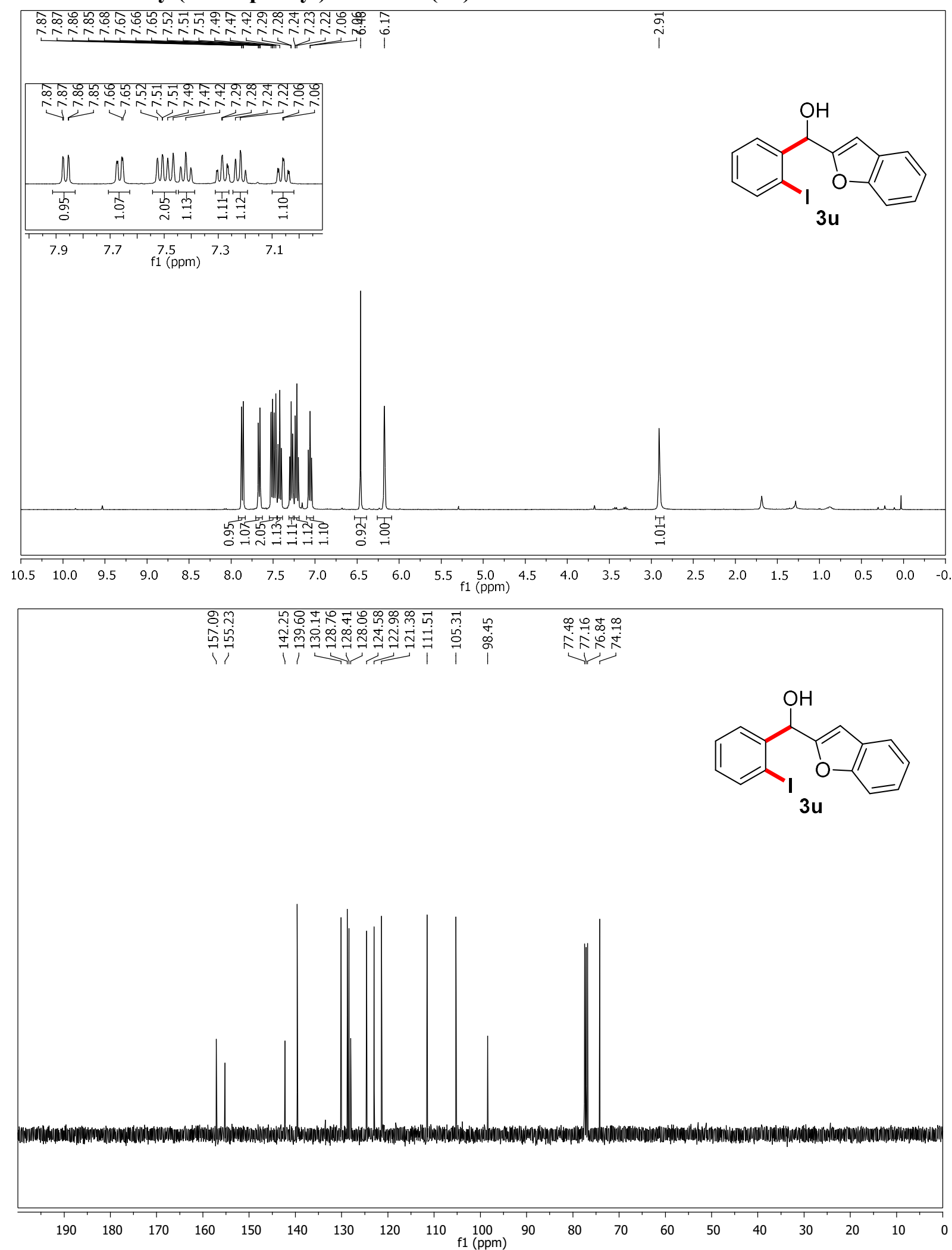


\section{Cyclohexyl(2-iodophenyl)methanol (3v)}

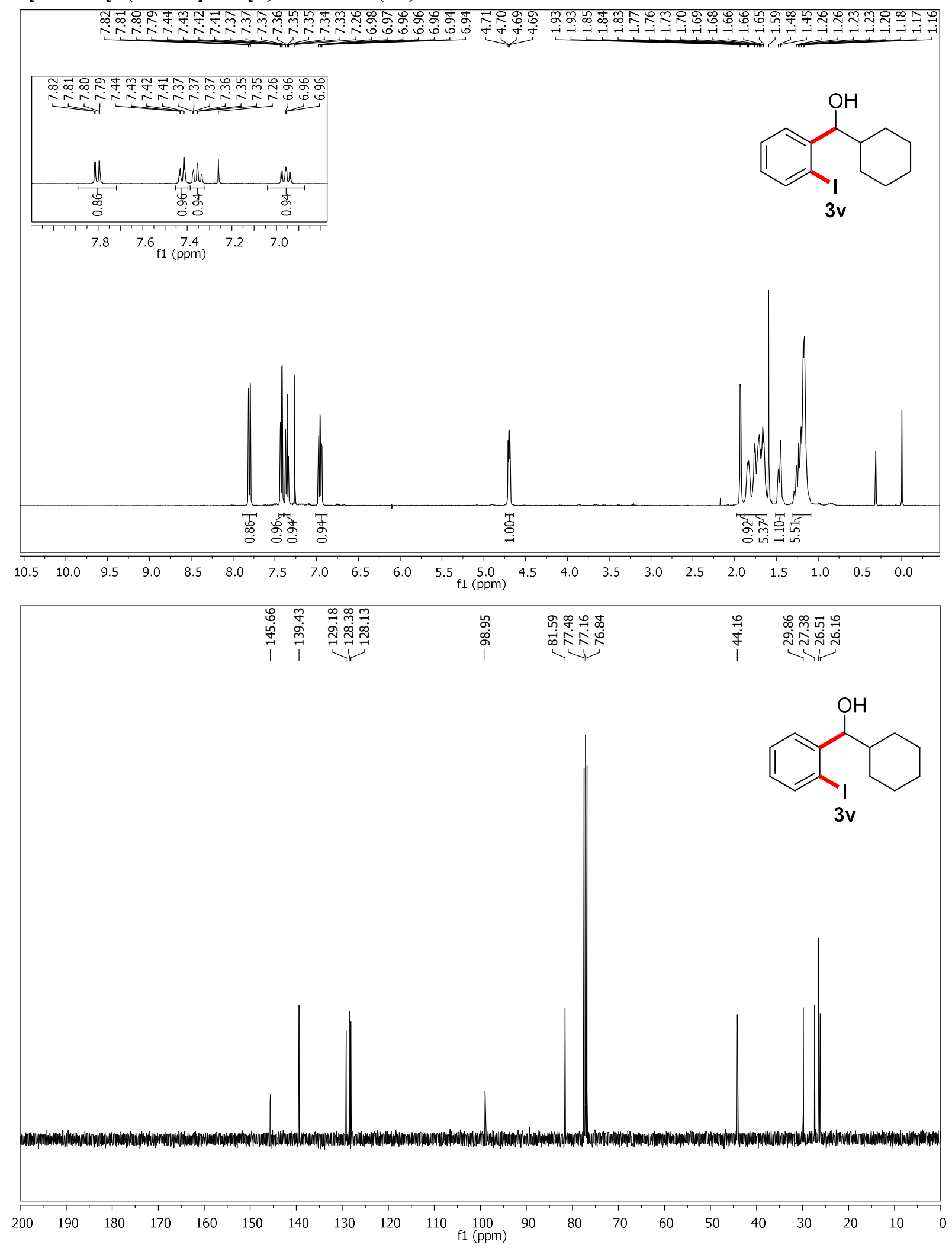




\section{1-(2-Iodophenyl)-2-methylpropan-1-ol (3w)}
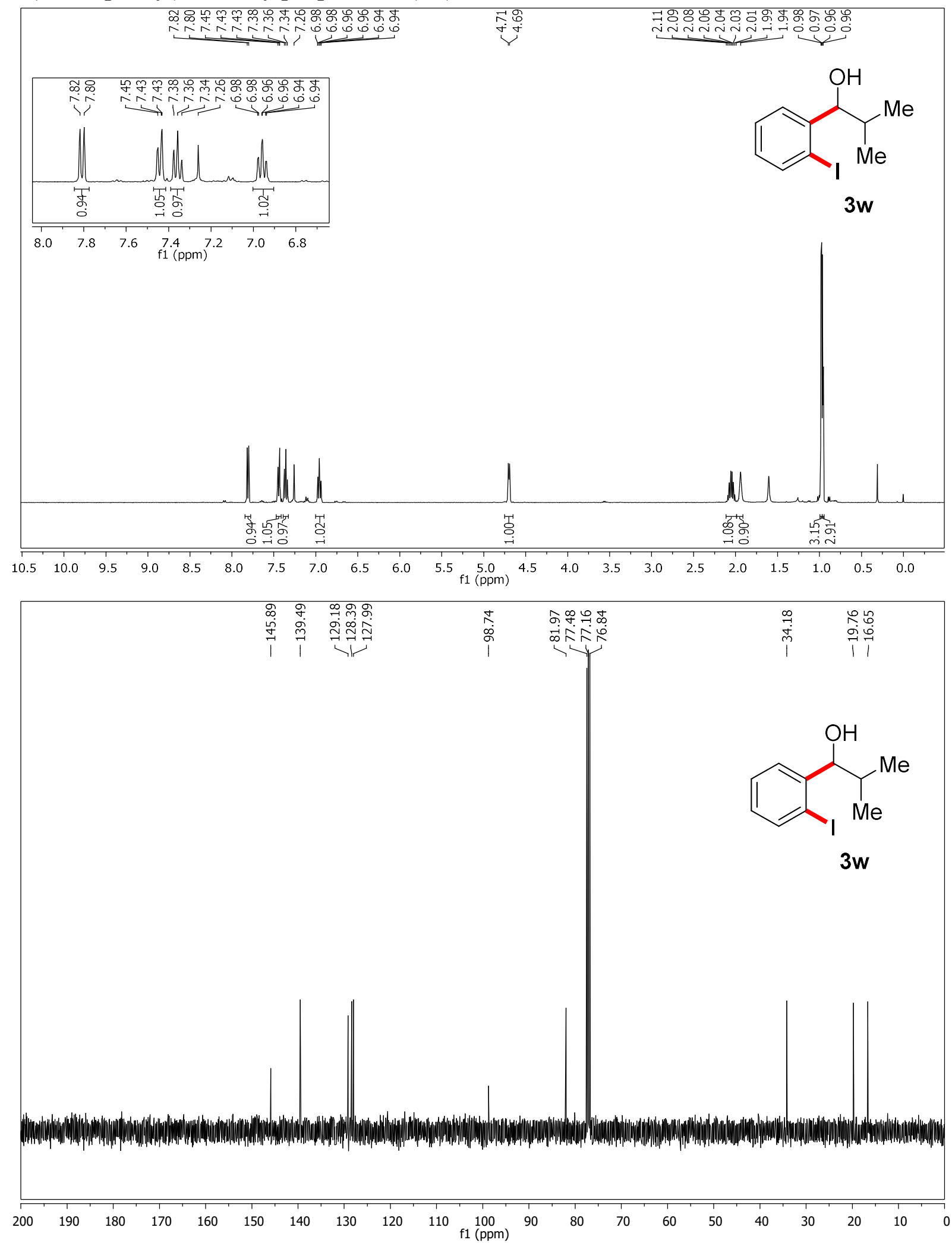
(2-Iodo-4,5-dimethylphenyl)(4-nitrophenyl)methanol (3x)
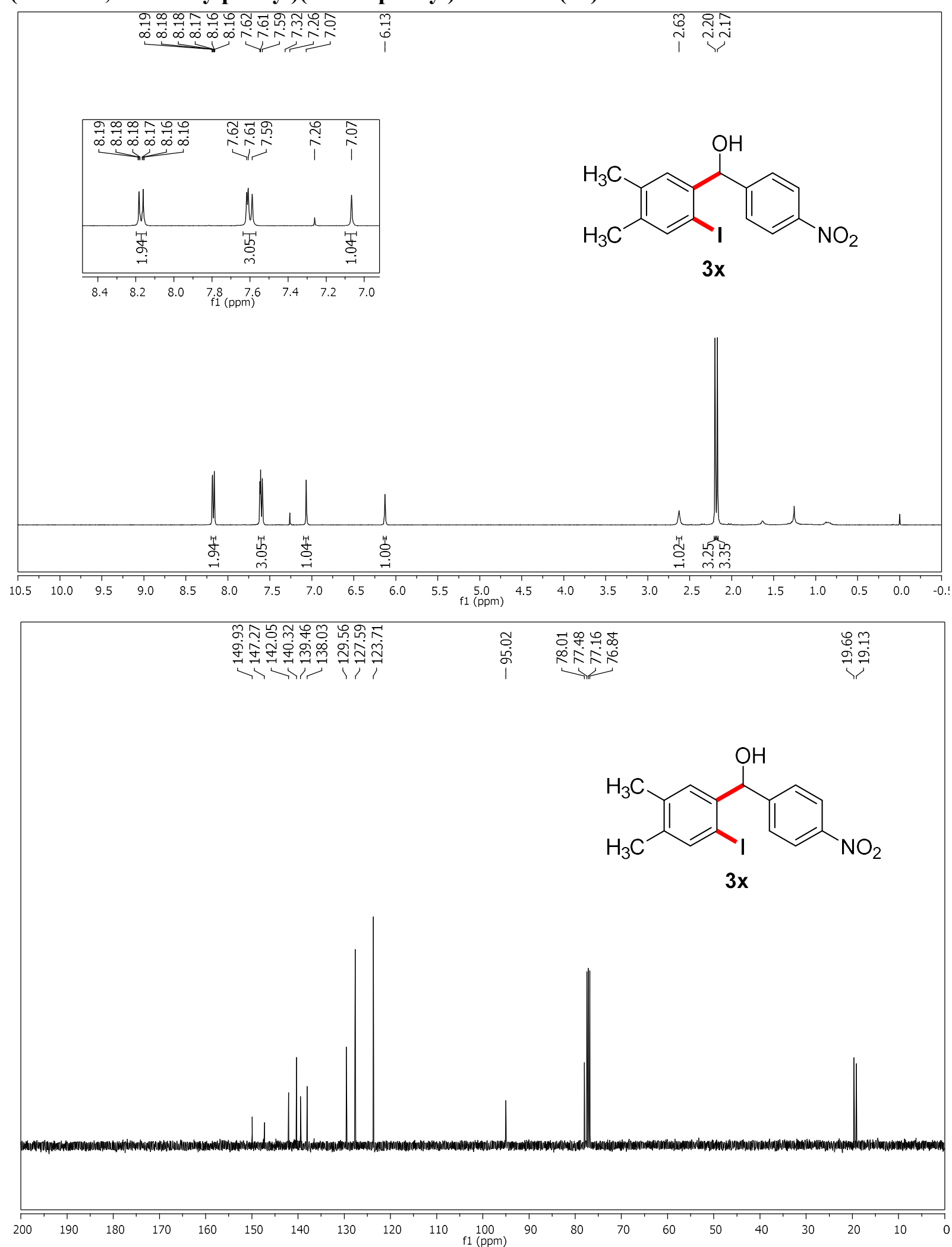
(6-Iodobenzo[d][1,3]dioxol-5-yl)(4-nitrophenyl)methanol (3y)
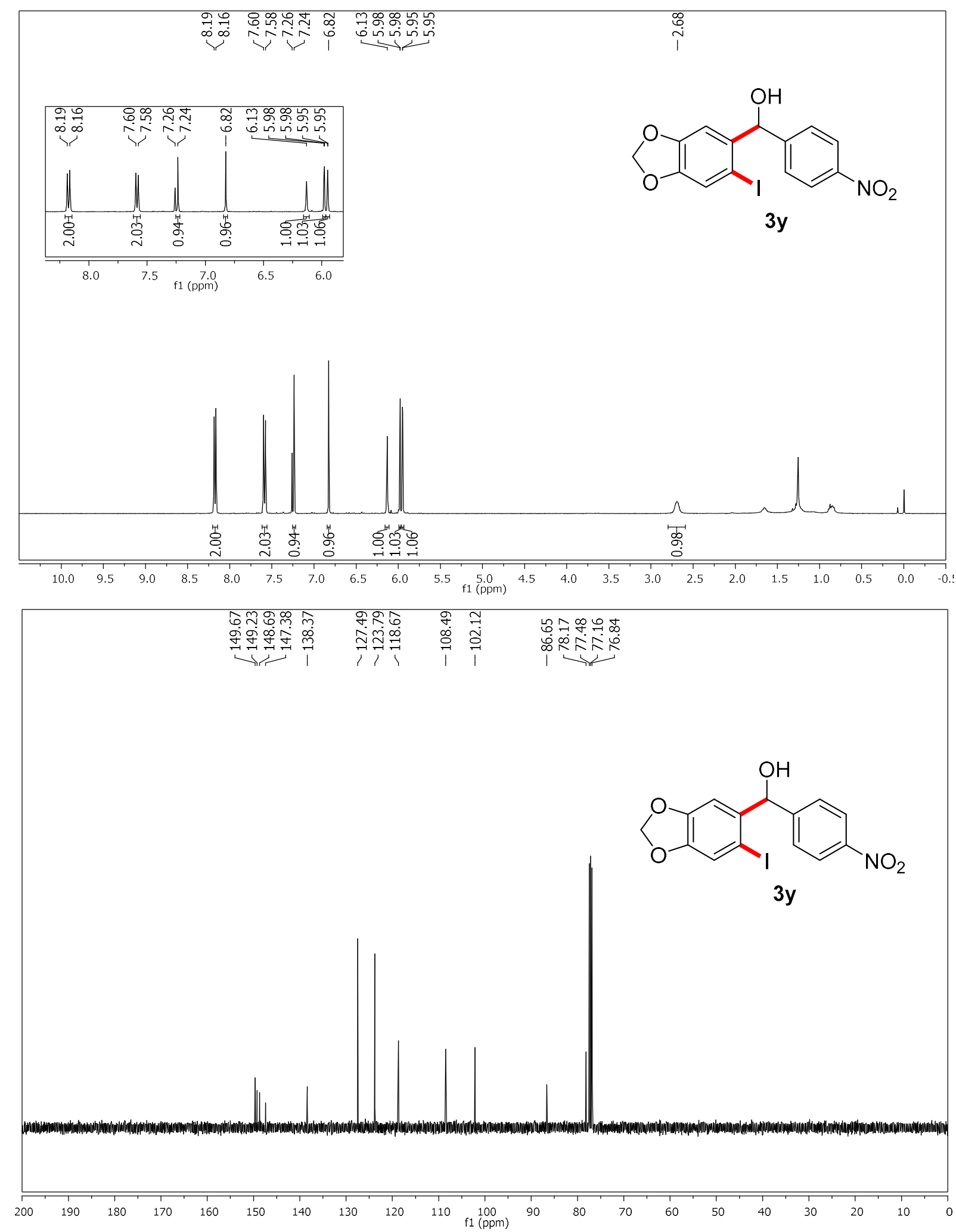
(2-Iodo-3,6-dimethylphenyl)(4-nitrophenyl)methanol (3z)
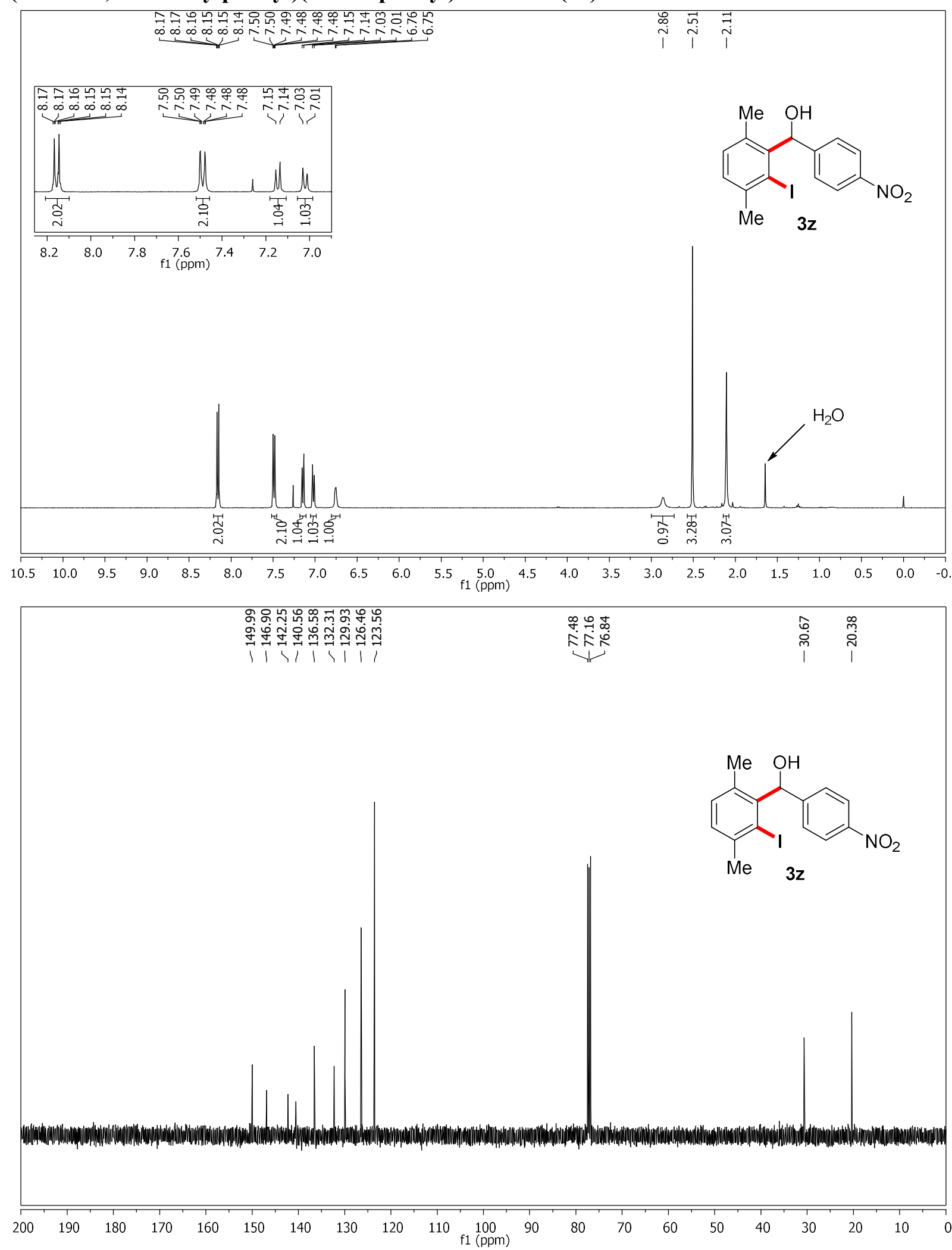
(3-Iodonaphthalen-2-yl)(4-nitrophenyl)methanol (3aa)
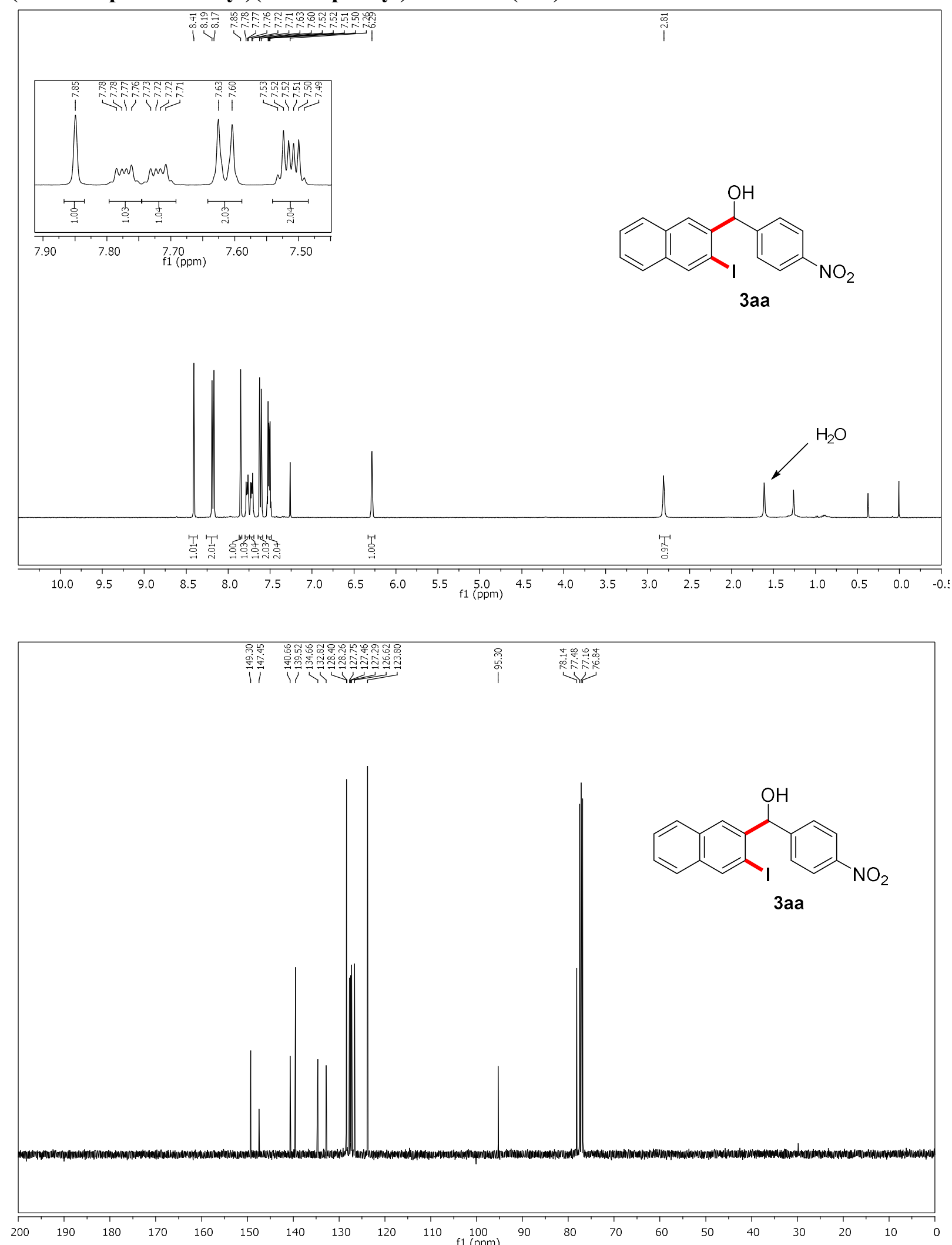
(2-Iodonaphthalen-1-yl)(4-nitrophenyl)methanol (3ab)
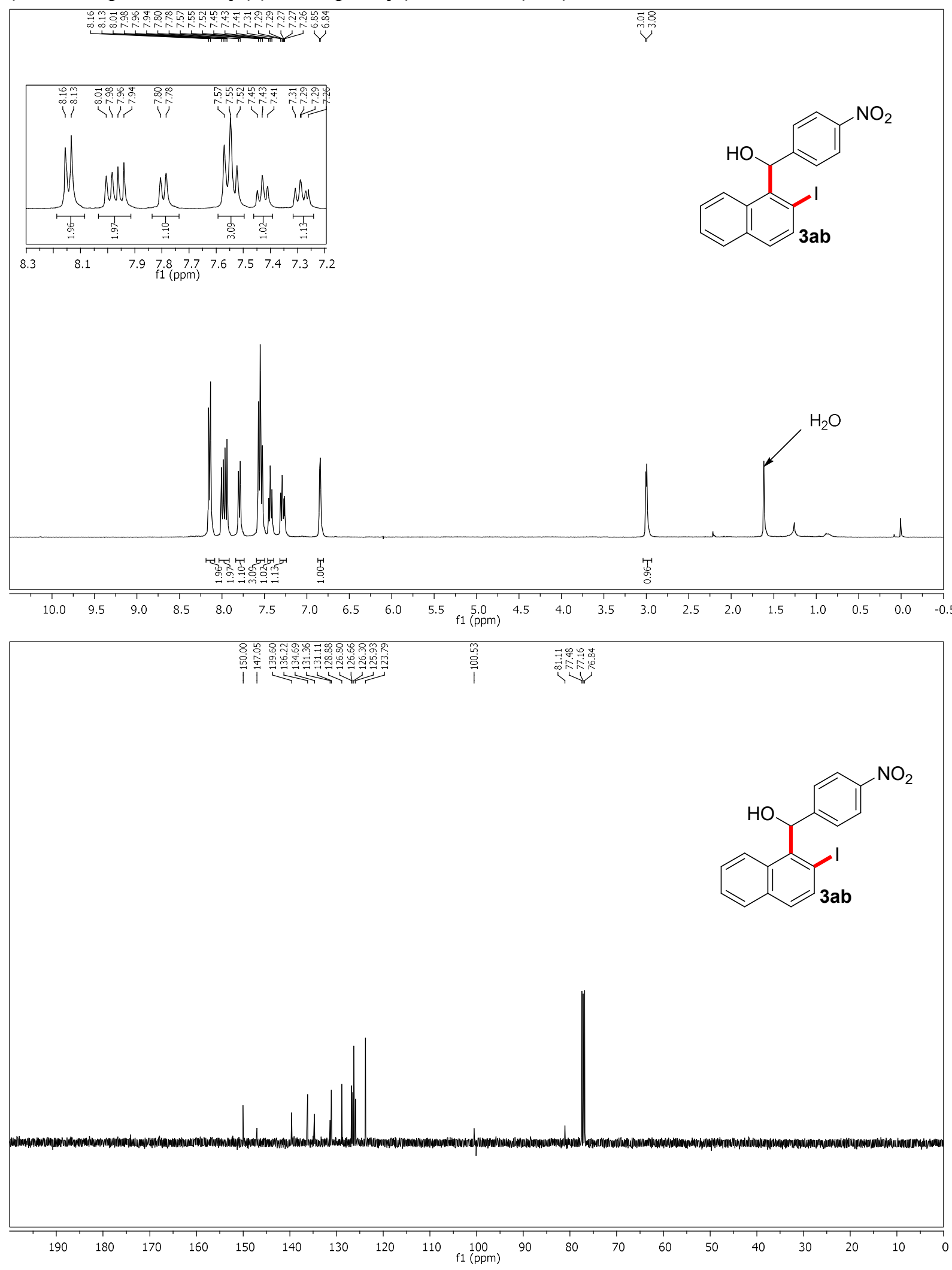
(2-Iodo-6-methoxyphenyl)(4-nitrophenyl)methanol (3ac)

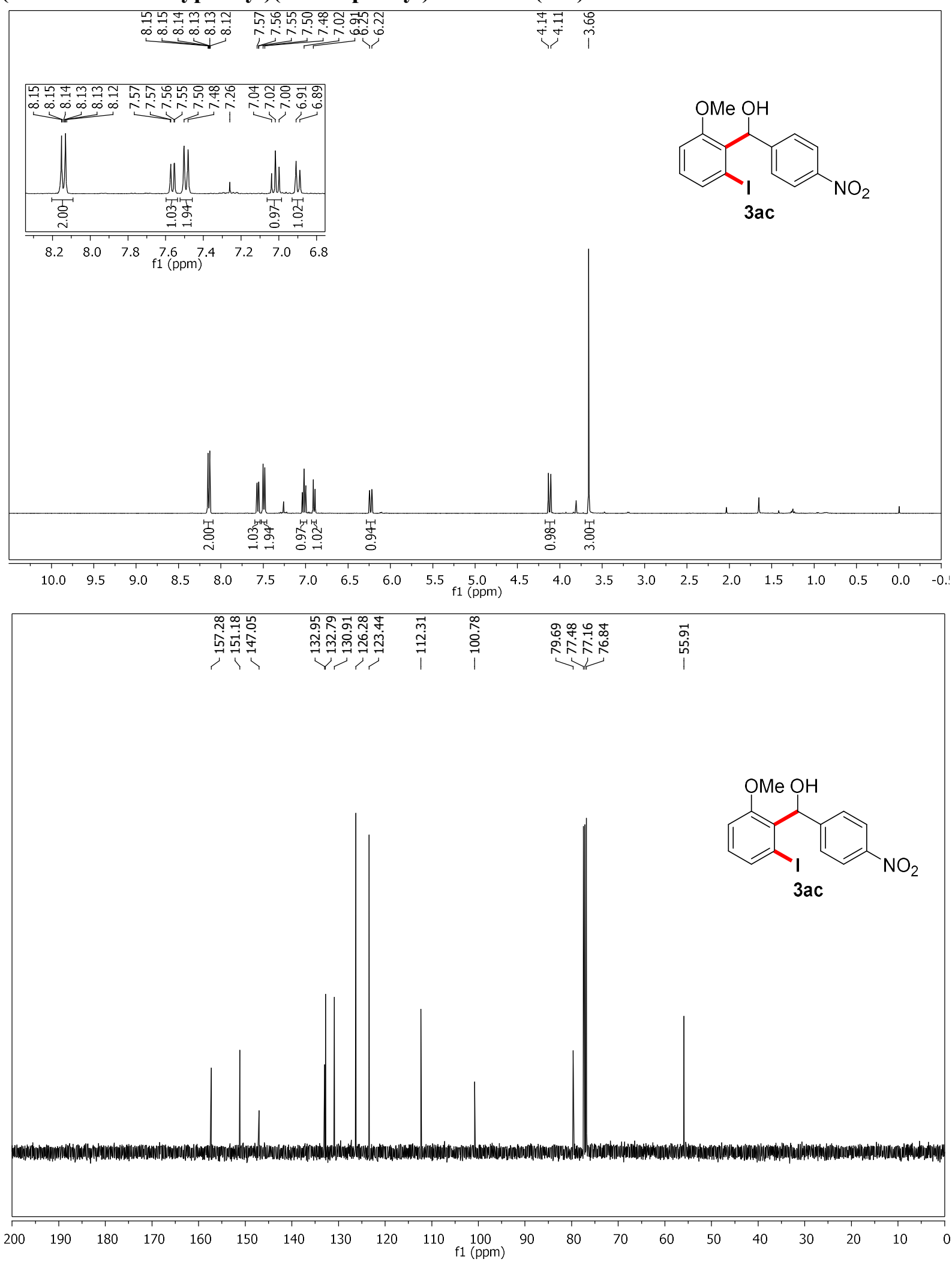


(2-Iodo-4-methylphenyl)(4-nitrophenyl)methanol and (2-Iodo-5-methylphenyl)(4nitrophenyl)methanol (3ad)
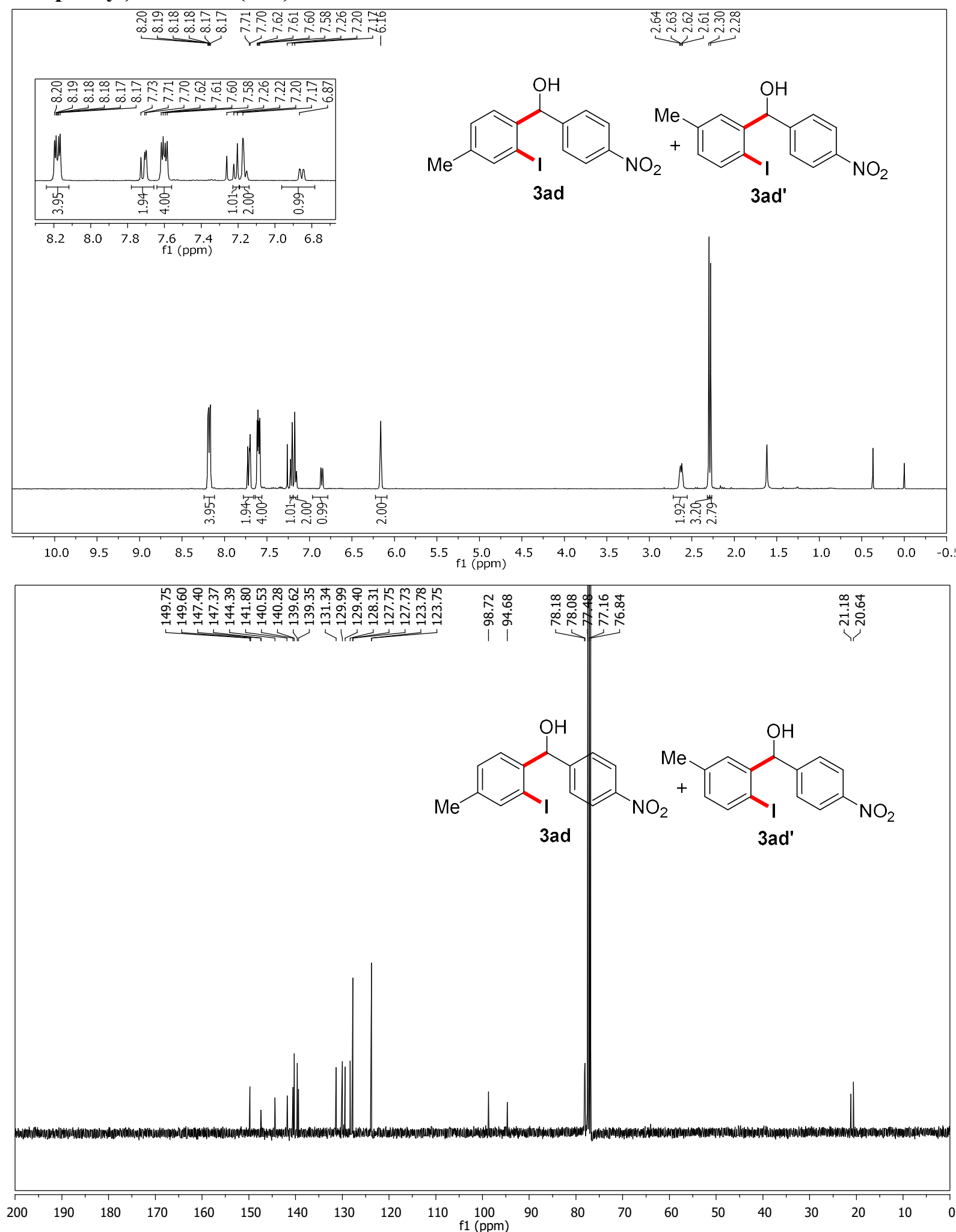
(2-Bromophenyl)(4-nitrophenyl)methanol (3ae)
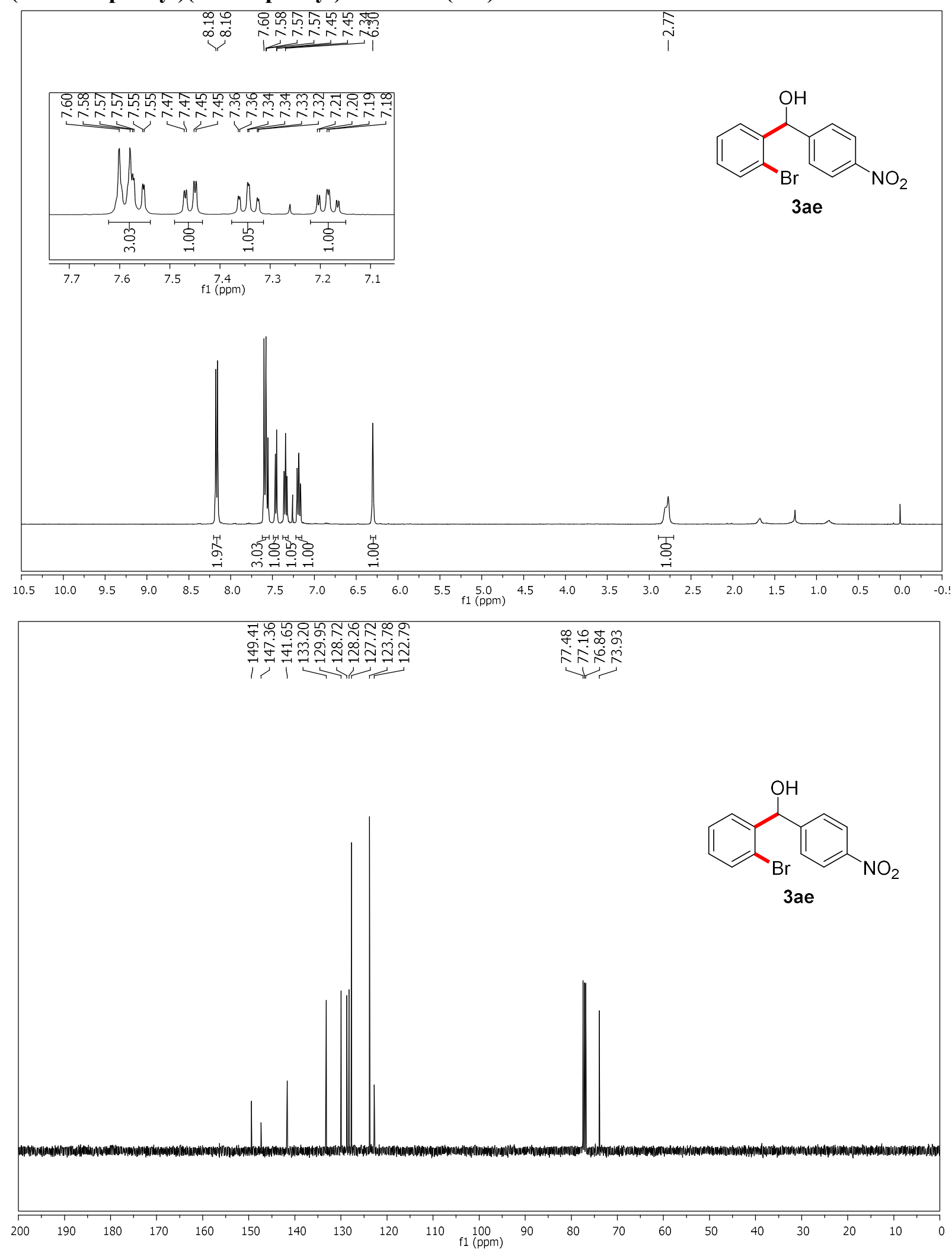
(2-Chlorophenyl)(4-nitrophenyl)methanol (3af)
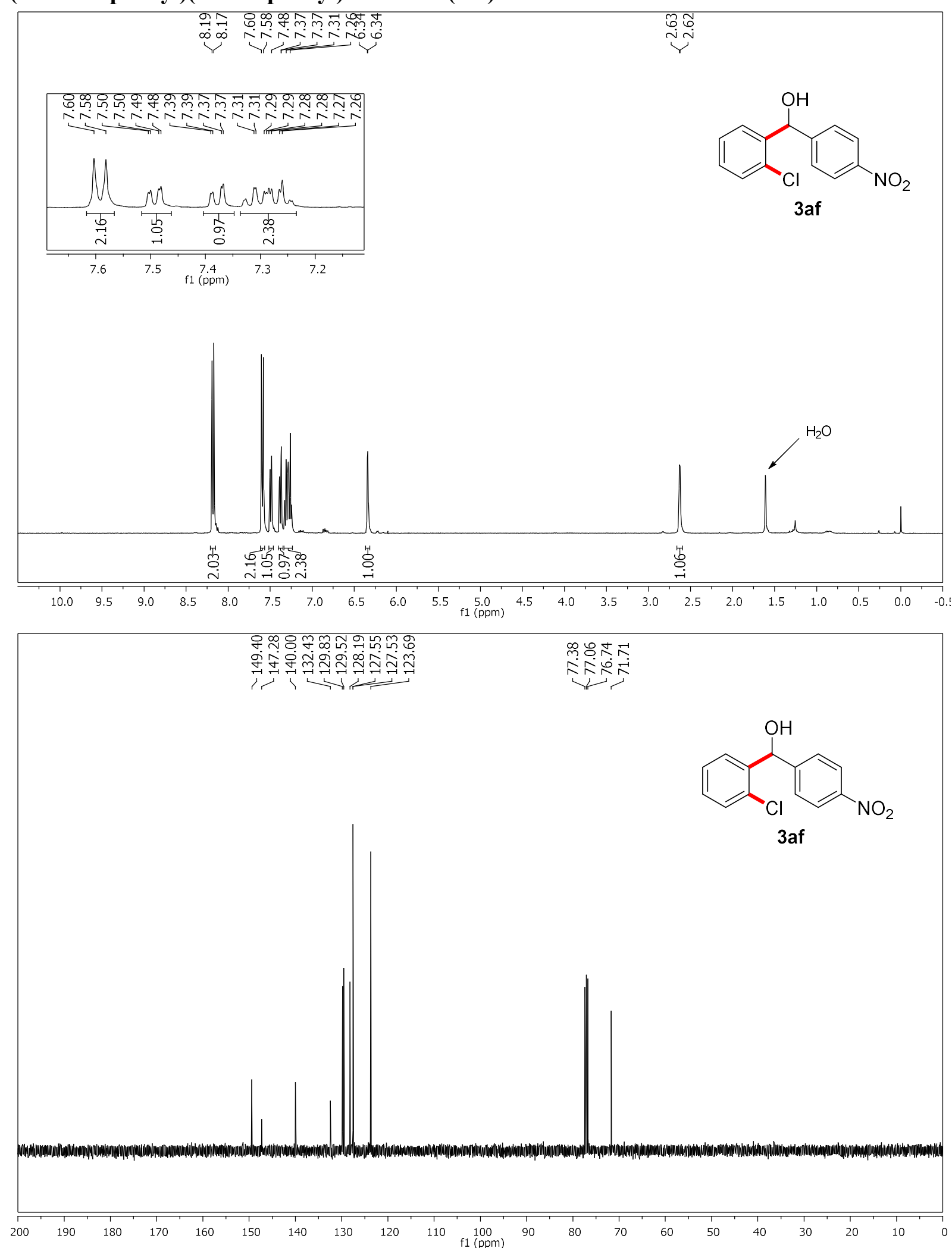


\section{(2-Bromophenyl)(phenyl)methanol (3ag)}
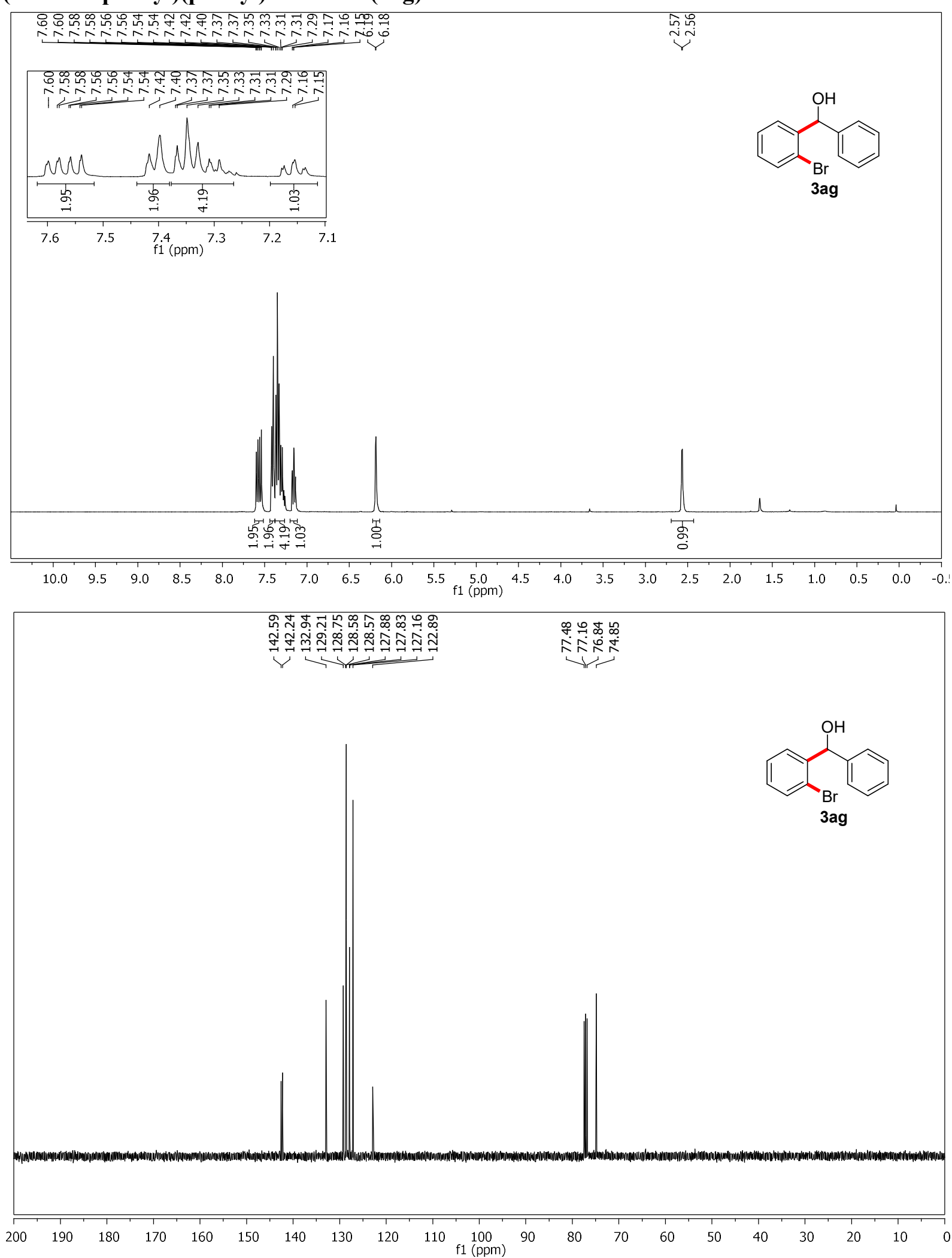
(2-Chlorophenyl)(phenyl)methanol (3ah)
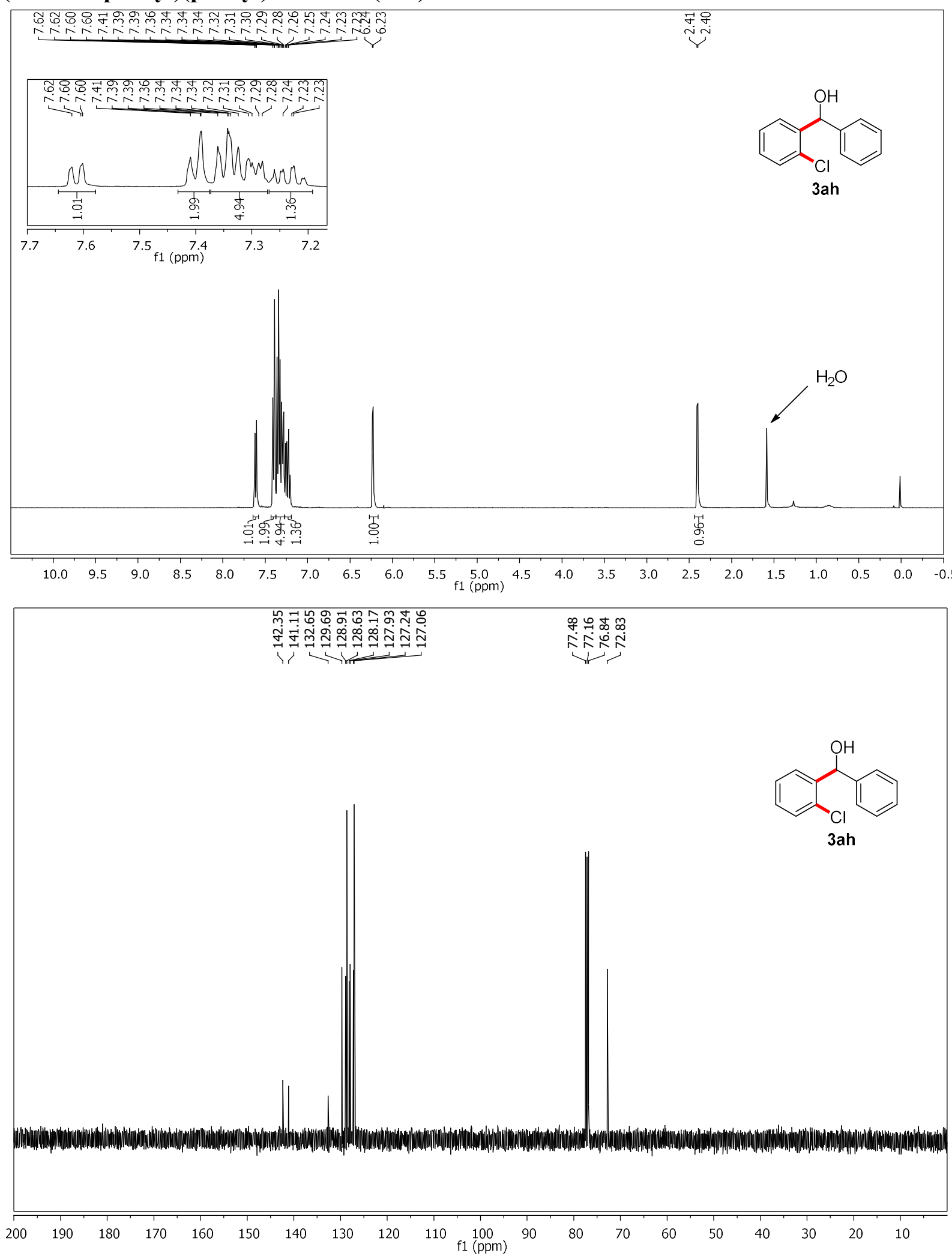
Benzofuran-2-yl(2-bromophenyl)methanol (3ai)
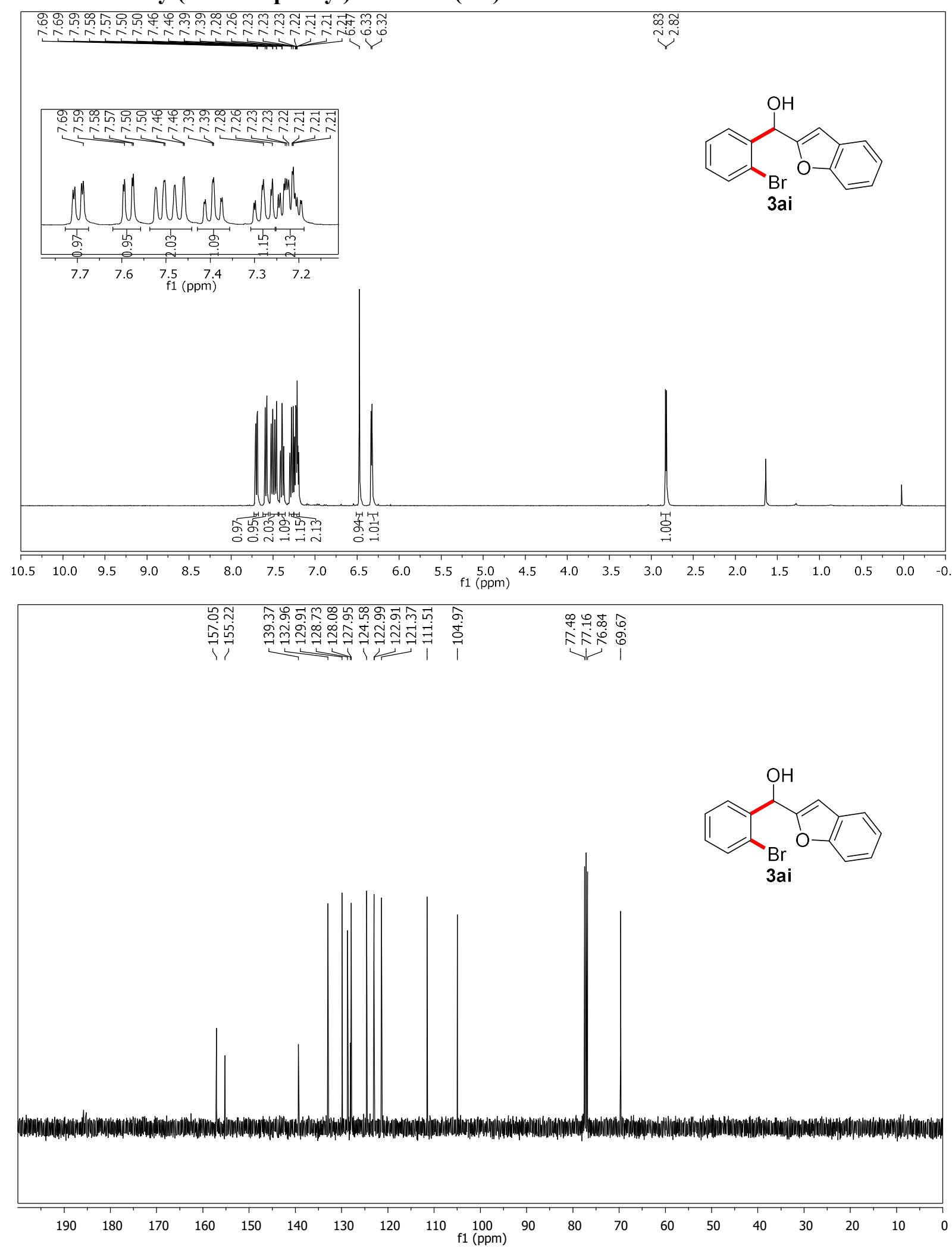
Benzofuran-2-yl(2-chlorophenyl)methanol (3aj)
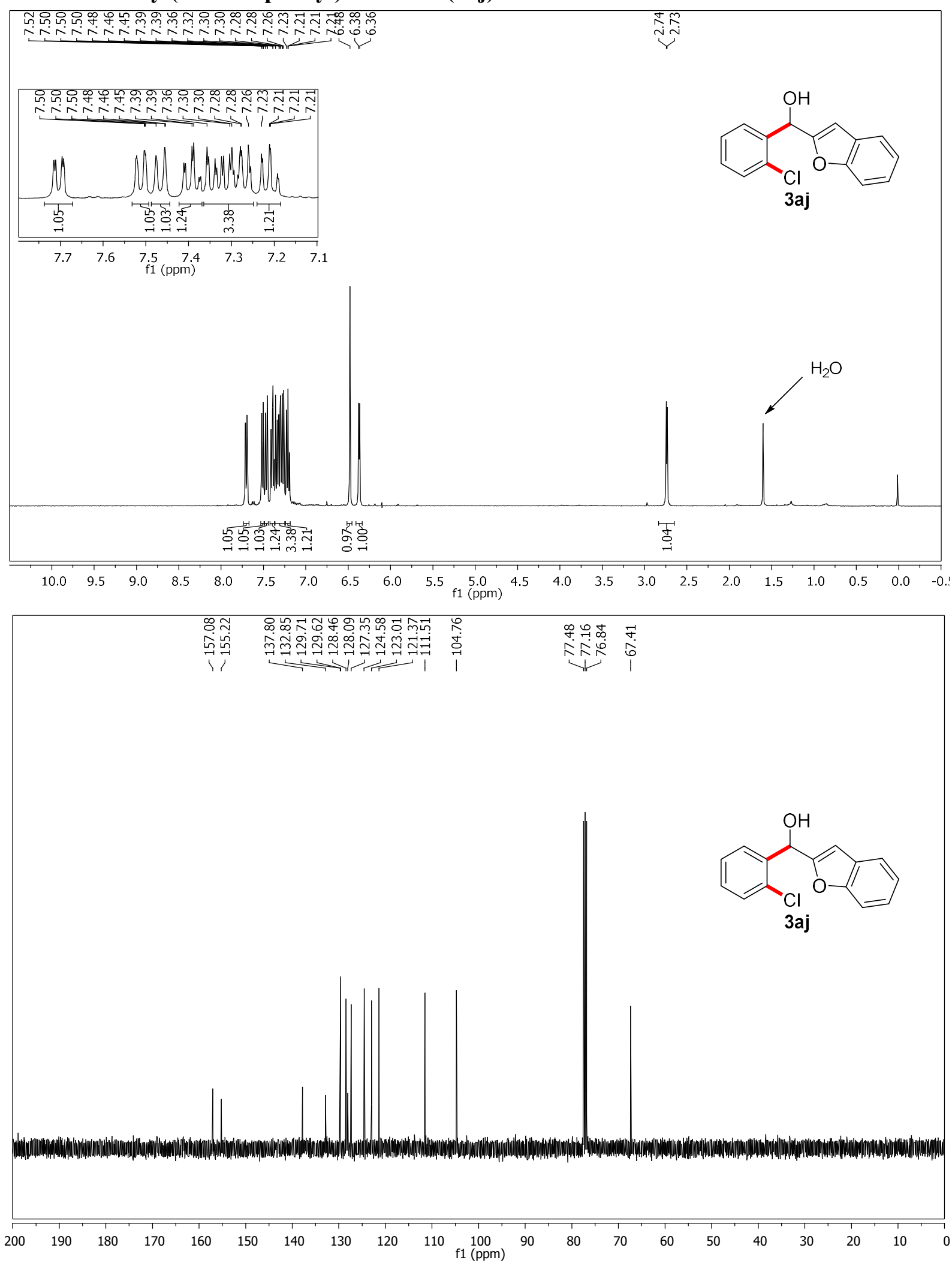


\section{1-(2-Bromophenyl)-2-methylpropan-1-ol (3ak)}
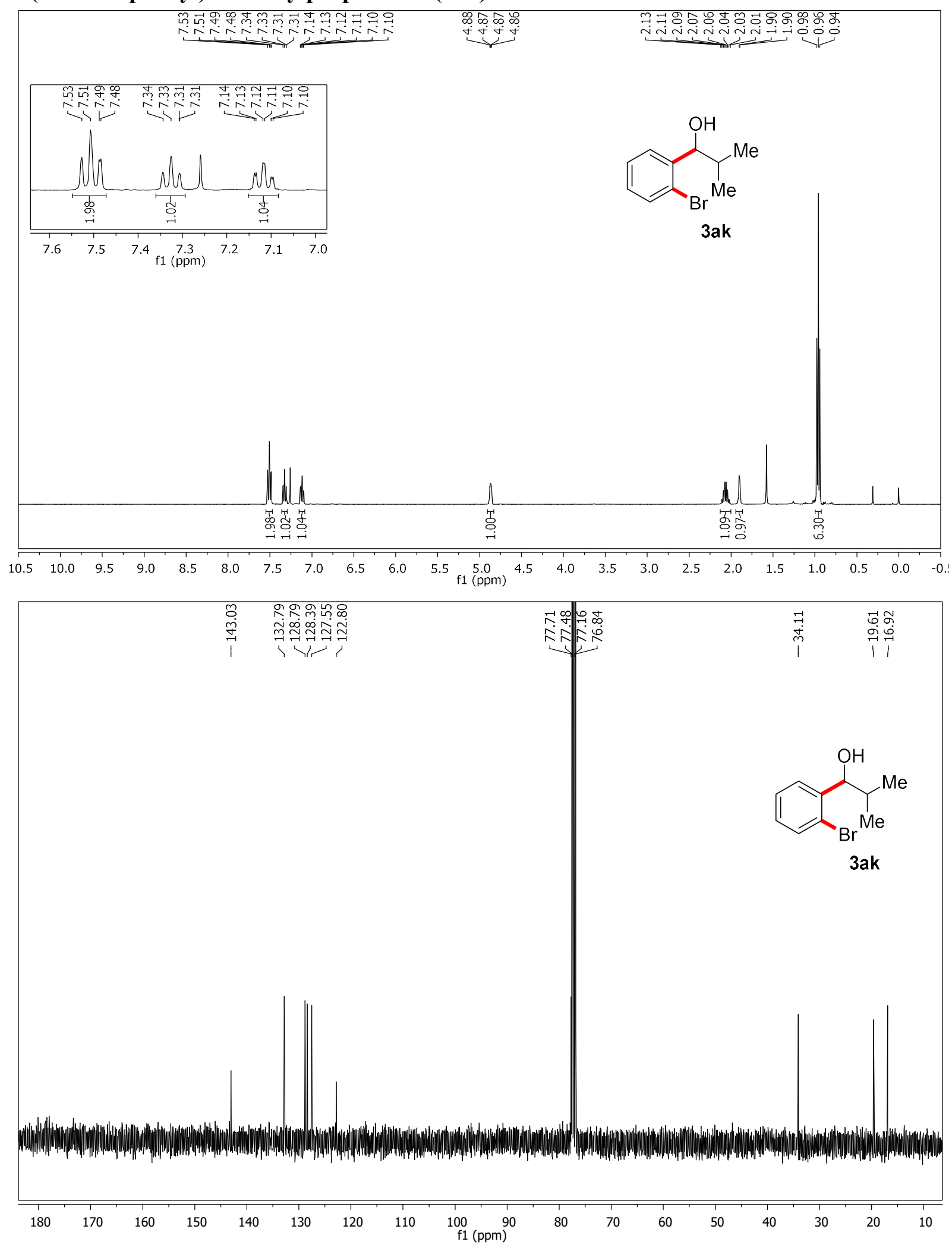
(6-Bromobenzo[d][1,3]dioxol-5-yl)(4-nitrophenyl)methanol (3al)
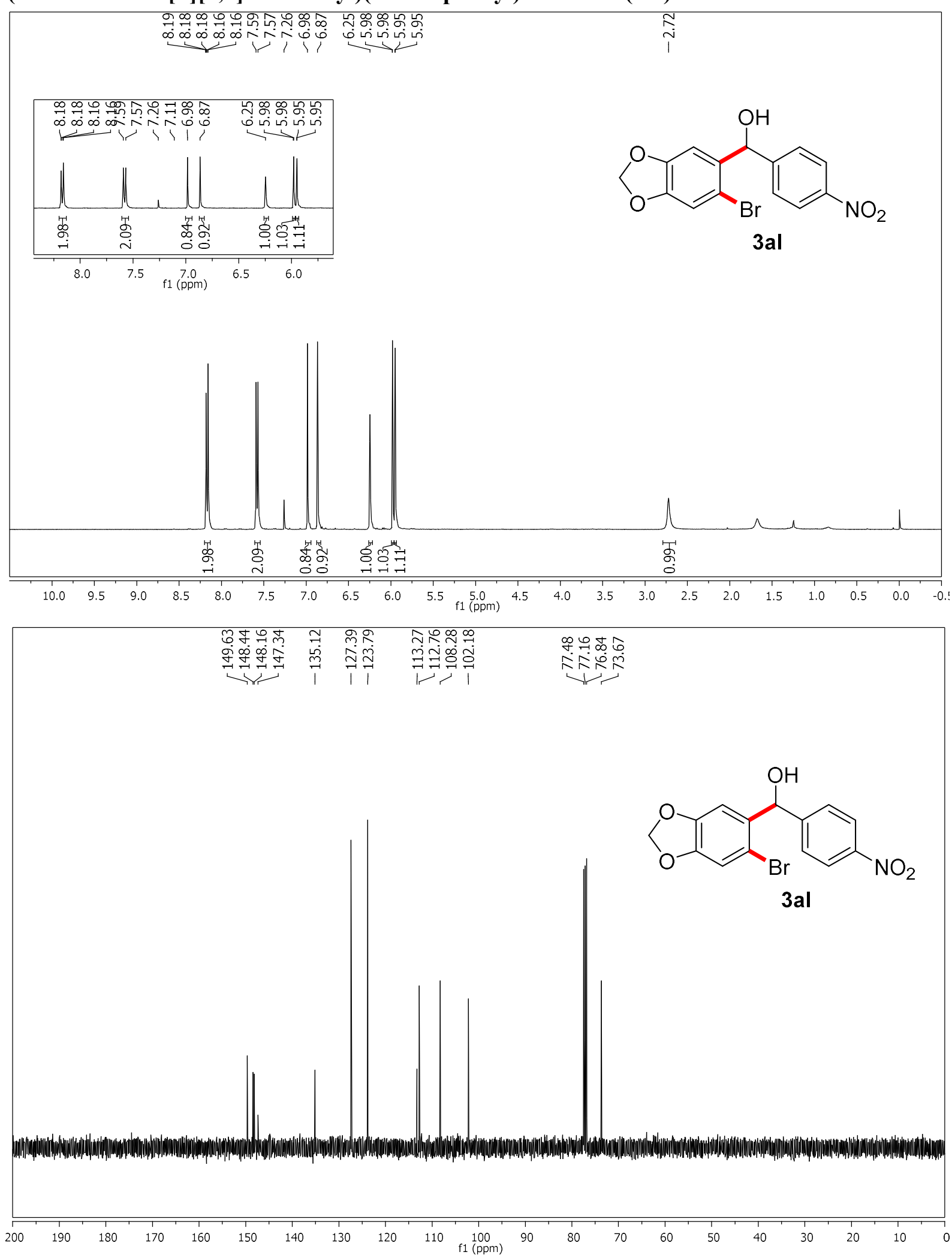
(6-Chlorobenzo[d][1,3]dioxol-5-yl)(4-nitrophenyl)methanol (3am)
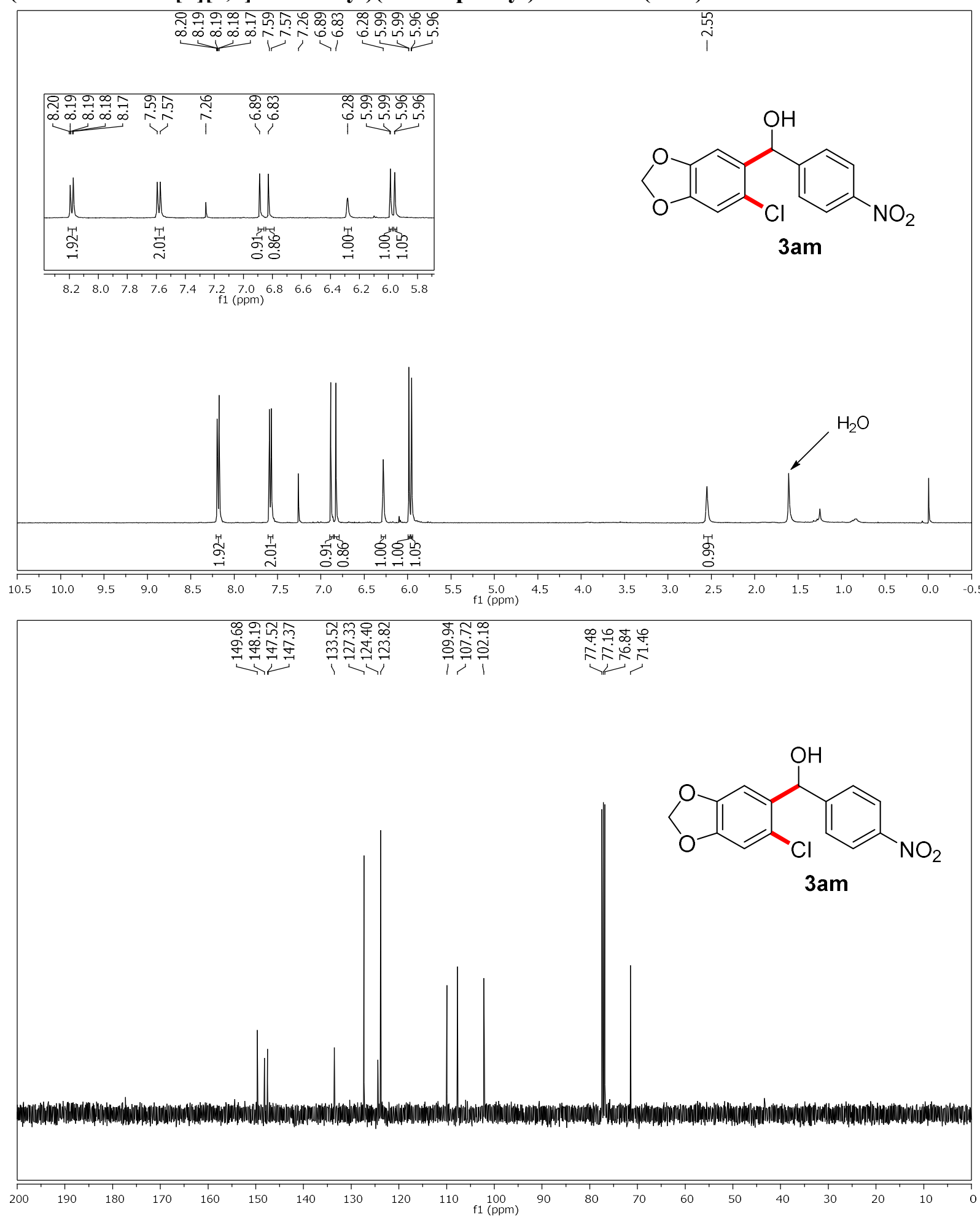
3-Hydroxy-3-(2-iodophenyl)-1-methylindolin-2-one (5a)
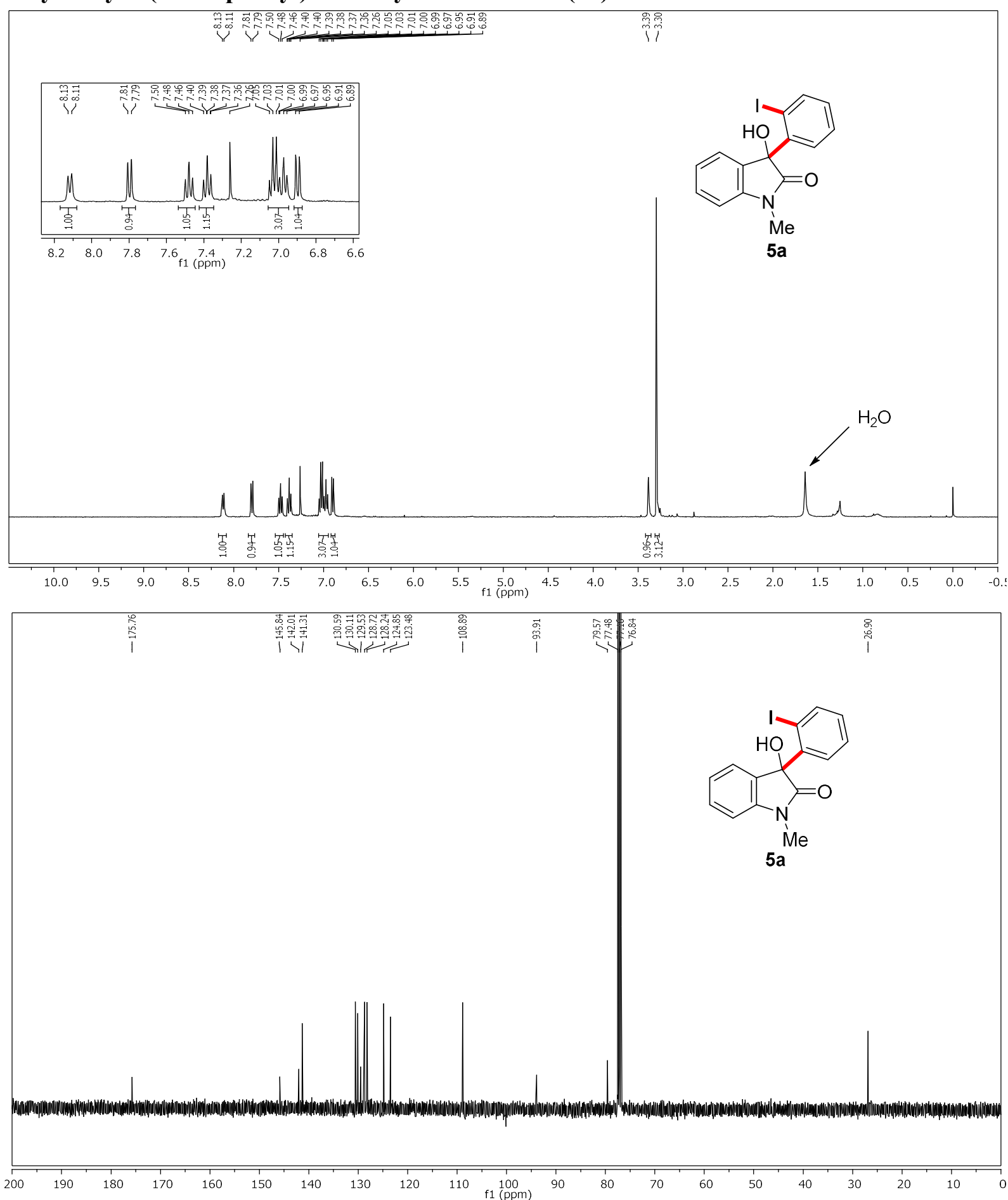
2-Iodobenzoic acid (6a)
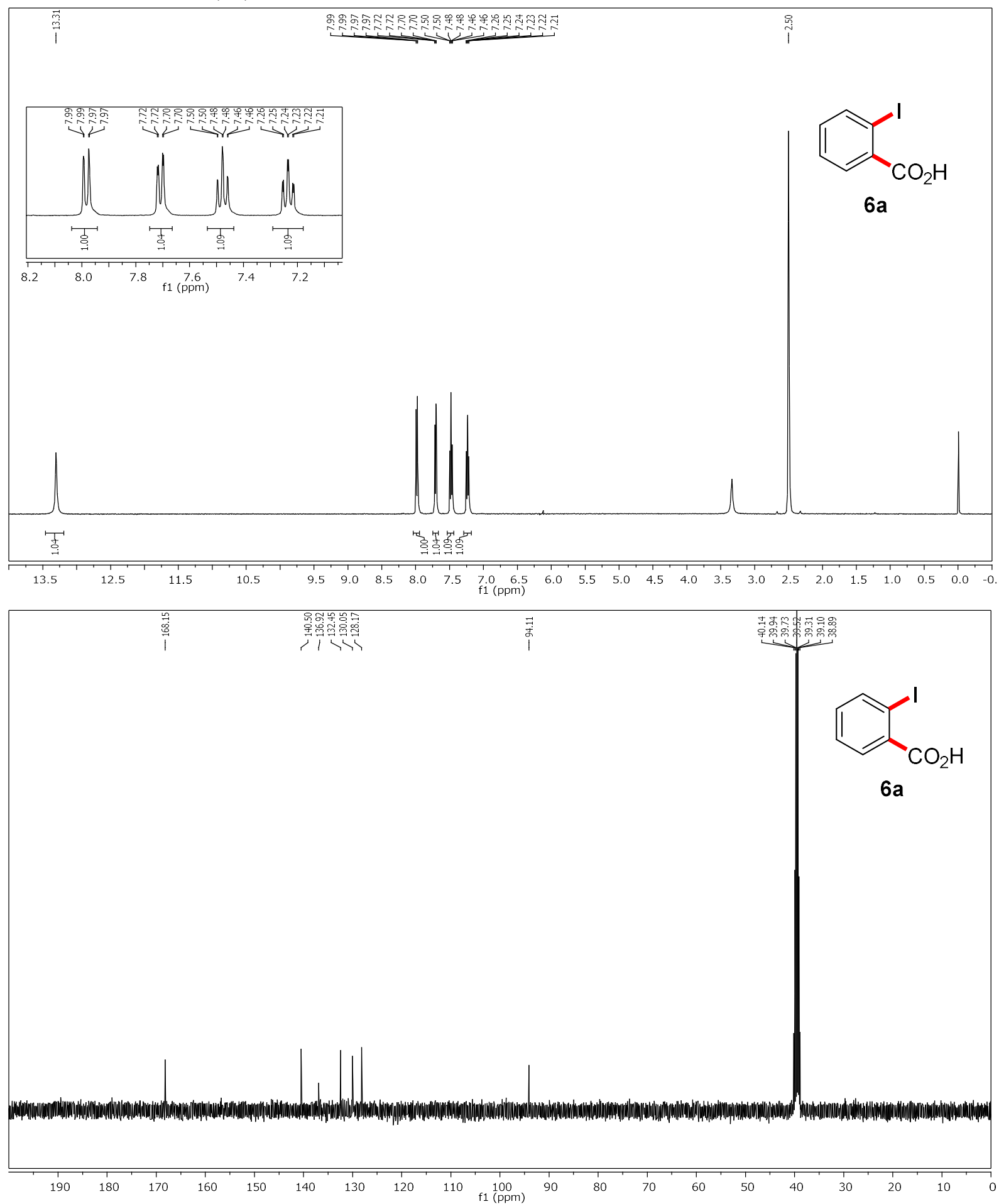
(4-Nitrophenyl)(2-(phenylethynyl)phenyl)methanol (7a)
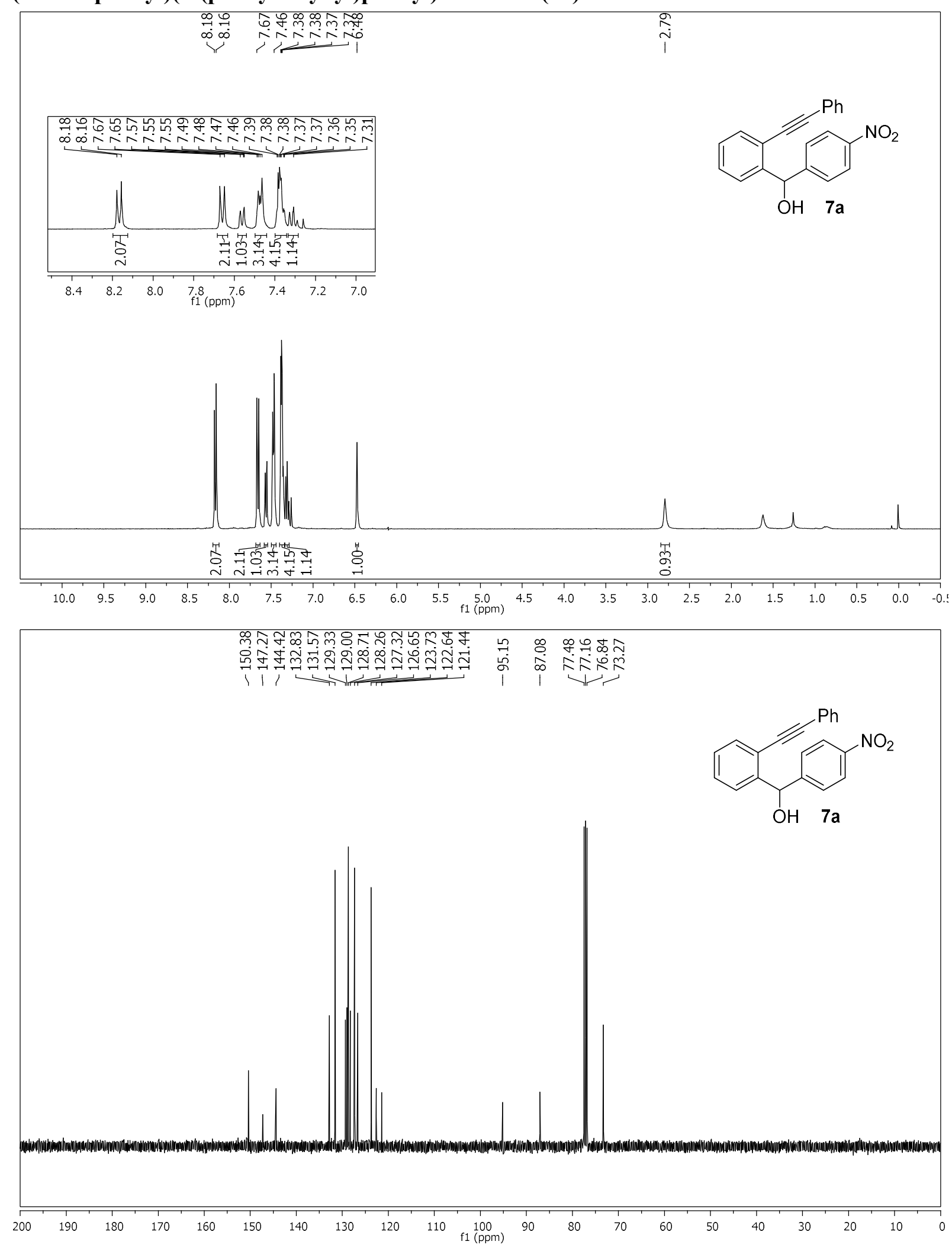
(Z)-1-Benzylidene-3-(4-nitrophenyl)-1,3-dihydroisobenzofuran (8a)
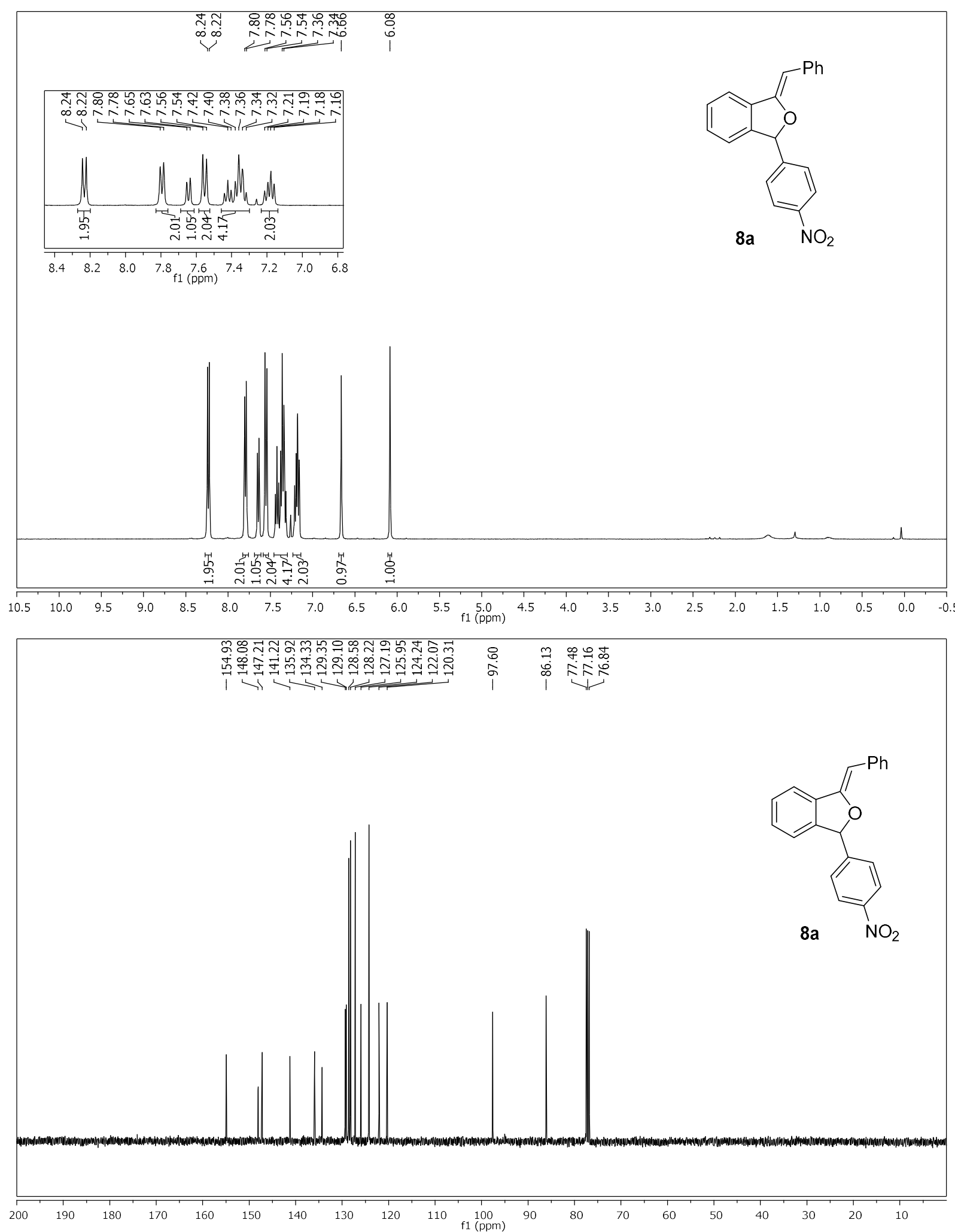
(Z)-1-Benzylidene-3-(4-nitrophenyl)-1,3-dihydroisobenzofuran (8b)
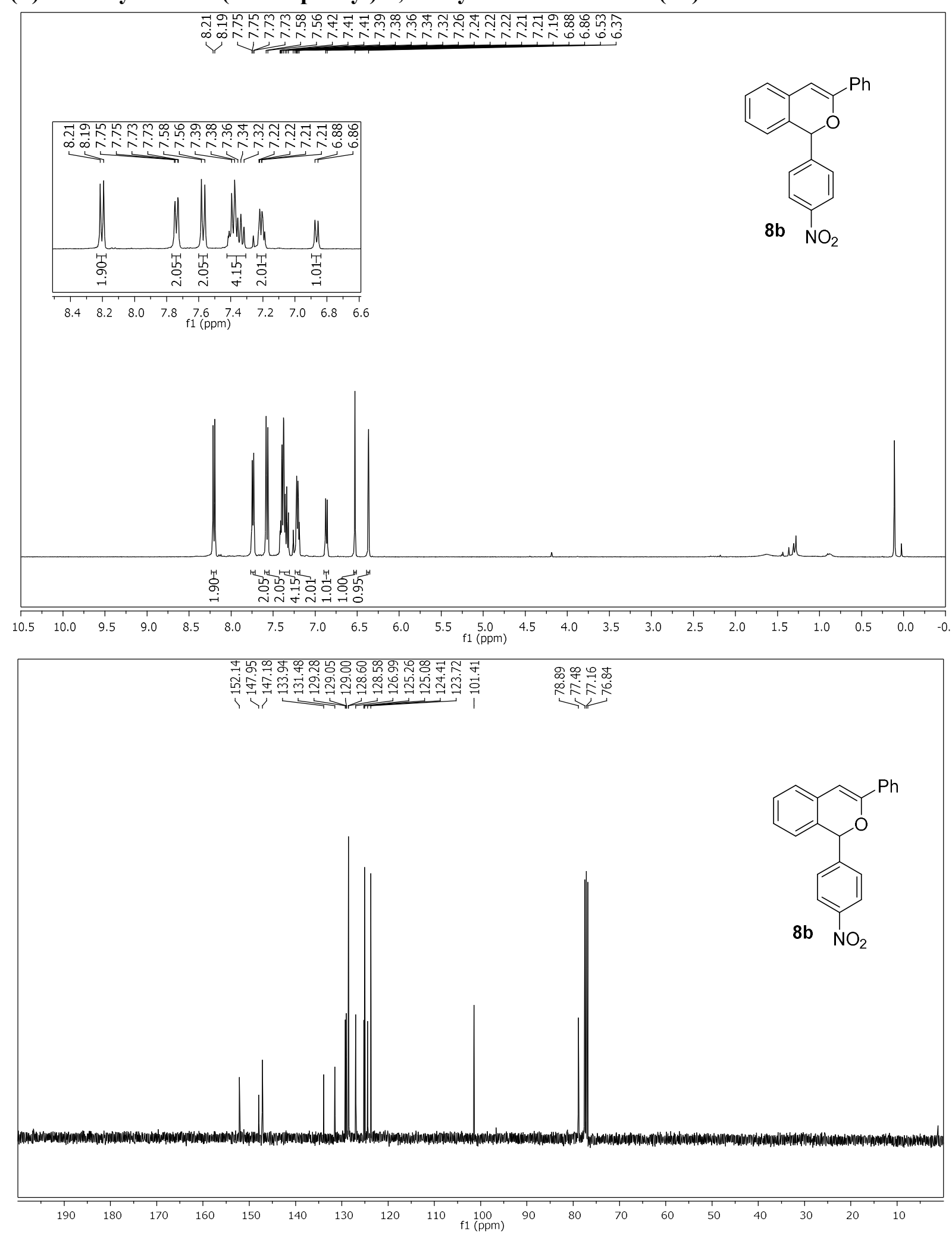
(E)-(4-Nitrophenyl)(2-styrylphenyl)methanol (9a)
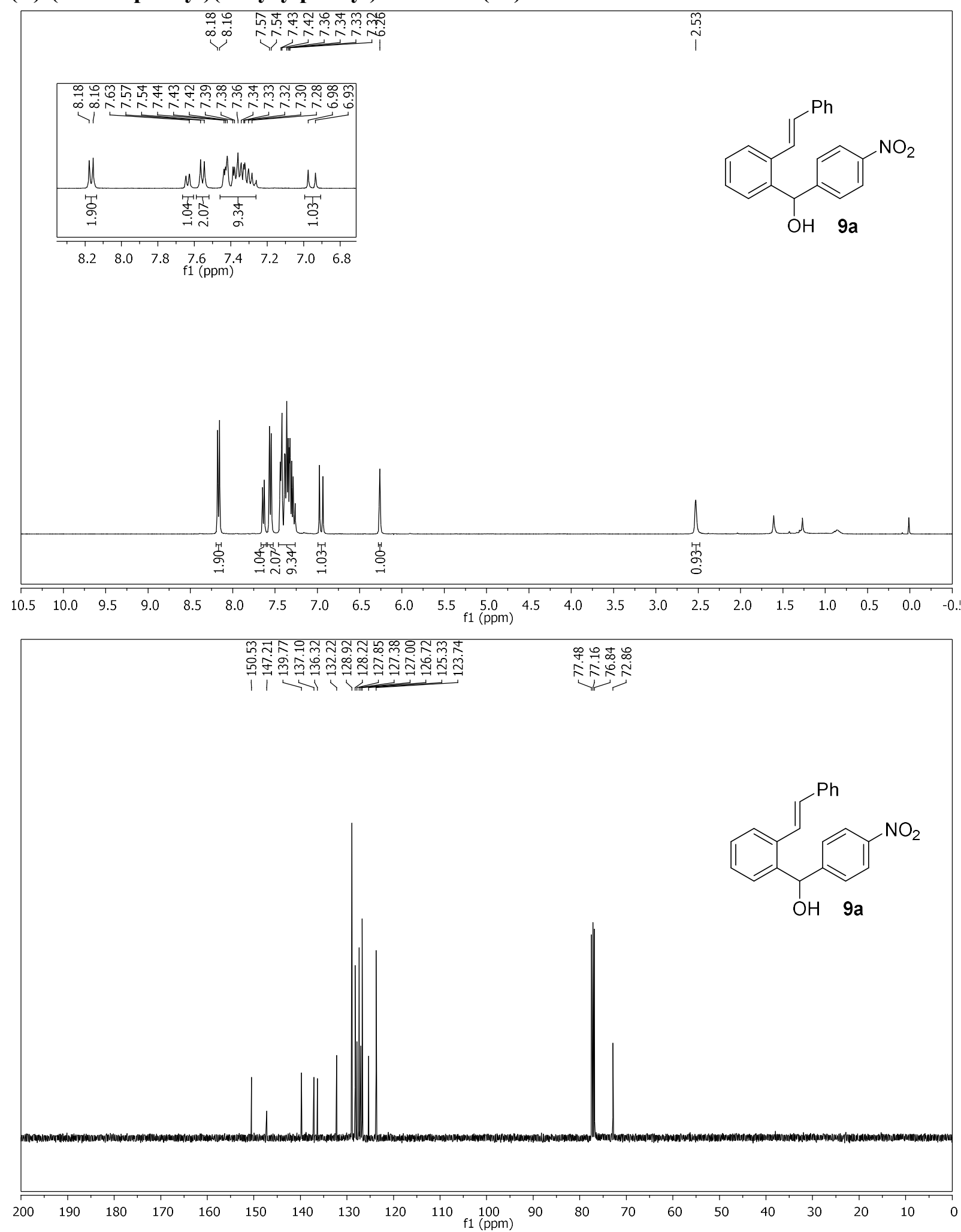
[1,1'-Biphenyl]-2-yl(4-nitrophenyl)methanol (10a)
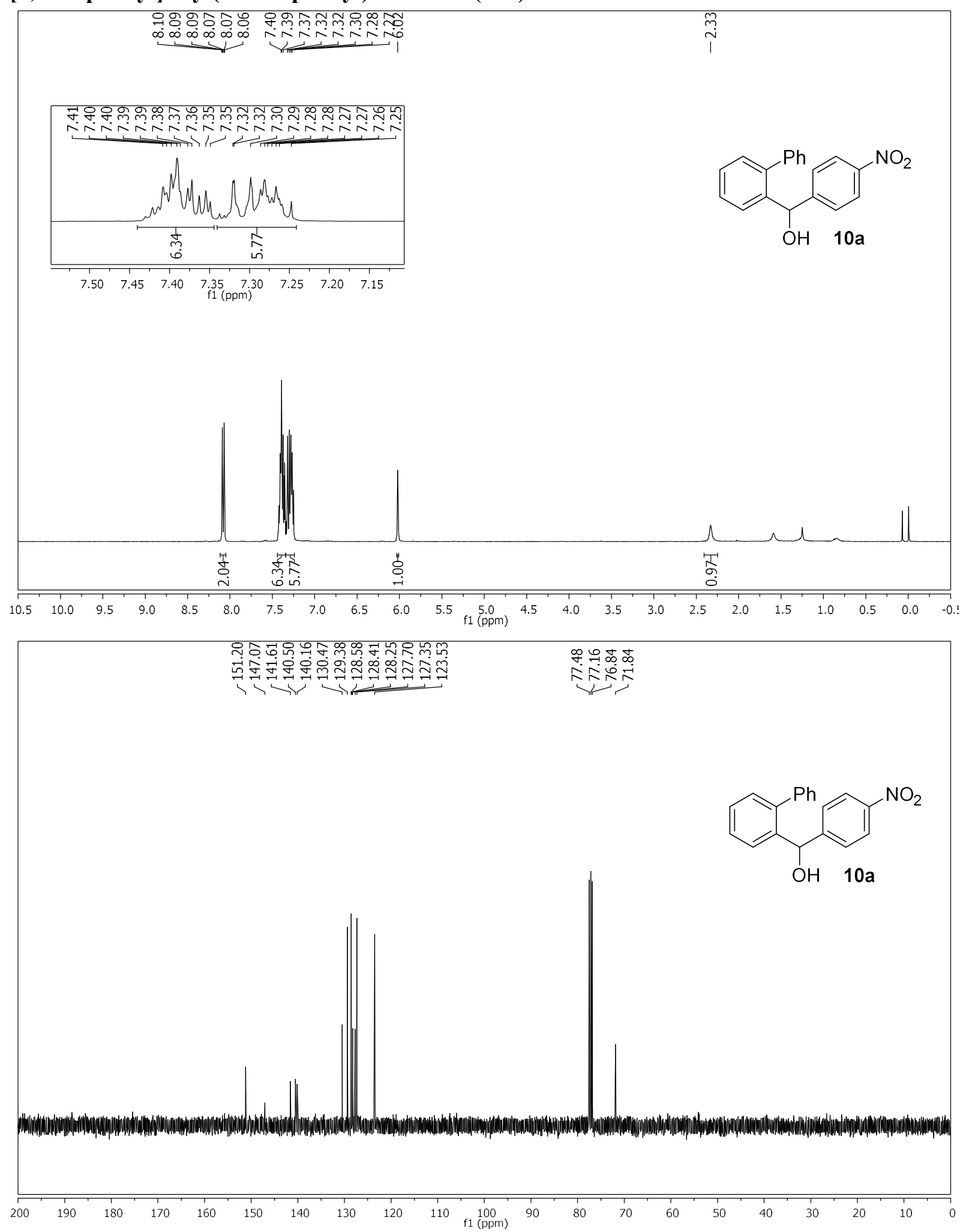